\title{
Oral and Electronic Poster Presentations 2018 Lumbar Spine Research Society Annual Scientific Meeting Chicago, Illinois • April 5-6, 2018
}

(DOI: 10.3171/2018.4.FOC.LSRSabstracts)

001. Transfusion of Older Blood Is Associated with Increased Perioperative Complications Among Spine Surgery Patients

Taylor Purvis, $B A^{1}$; Zach Pennington, B.S. ${ }^{2}$, C. Rory Goodwin, MD, PhD ${ }^{3}$, Camilo Molina, MD $^{4}$, Steven Frank, MD ${ }^{4}$, Daniel Sciubba, MD ${ }^{5}$

${ }^{1}$ Johns Hopkins School of Medicine, Baltimore, MD , ${ }^{2}$ Johns Hopkins University, Baltimore, MD ${ }^{3}$, Durham, NC, ${ }^{4}$, Baltimore, MD,${ }^{5}$ Johns Hopkins Hospital, Baltimore, Maryland

Background/Introduction: Despite retrospective studies that have shown that longer packed red blood cell (PRBC) storage duration worsens patient outcomes, randomized clinical trials have found no difference in outcomes. However, no studies have examined the impact of giving the oldest blood (28 days-old or more) on morbidity within spine surgery. Our objective in this study was to describe the association between storage duration of PRBCs and perioperative adverse events in patients undergoing spine surgery at a tertiary care center.

Materials/Methods: The surgical administrative database at our institution was queried for patients transfused with PRBCs who underwent spine surgery between December 4, 2008 and June 26, 2015. Patients undergoing spinal fusion, tumor-related surgeries, and other identified spine surgeries were included. Patients were divided into two groups based on storage duration of blood transfused: exclusively $\leq 28$ days' storage or exclusively $>28$ days' storage. The primary outcome was composite in-hospital morbidity, which included: (1) infection, (2) thrombotic event, (3) renal injury, (4) respiratory event, and/or (5) ischemic event.

Results: In total, 1,141 patients who received a transfusion were included for analysis in this retrospective study; 710 were transfused exclusively with PRBCs $\leq 28$ days' storage and 431 exclusively with PRBCs $>28$ days' storage. Perioperative complications occurred in 119 patients (10.4\%). Patients who received blood stored for $>28$ days had higher odds of developing any one complication (odds ratio [OR] $=1.82 ; 95 \%$ confidence interval $[\mathrm{CI}], 1.20-2.74 ; \mathrm{P}=0.005)$ even after adjusting for competing perioperative risk factors.

Discussion/Conclusion: Blood stored for $>28$ days is independently associated with higher odds of developing perioperative complications in patients transfused during spinal surgery. Our results suggest that blood storage duration may be an appropriate parameter to consider when developing institutional transfusion guidelines that seek to optimize patient outcomes.

002. Risk Factors Associated with 90-day Readmissions after Degenerative Lumbar Fusion: An Examination of the Michigan Spine Surgery Improvement Collaborative (MSSIC) Registry

Ilyas Aleem, MD, MSc, FRCSC ${ }^{1}$, Paul Park, $M D^{2}$, David Nerenz, PhD ${ }^{3}$, Lonni Schultz, MD ${ }^{3}$, Michael Bazydlo, MD ${ }^{3}$, Shujie Xiao, MD ${ }^{3}$, Hesham Zakaria, $M D^{3}$, Jason Schwalb, $M D^{3}$, Victor Chang, $M D^{3}$

${ }^{l}$ University of Michigan, Ann Arbor, MI, ${ }^{2}$, Ann Arbor, Michigan, ${ }^{3}$, Detroit, MI

Background/Introduction: Hospital readmission following spinal surgery negatively impacts the patient experience and significantly increases healthcare costs. To date, most studies have evaluated 30-day readmissions after spine surgery. Evaluation of the 90-day period, however, allows a more comprehensive assessment of factors associated with readmission. The purpose of this study is to assess the causes and risk factors for 90 -day hospital readmissions after lumbar fusion surgery.

Materials/Methods: We performed a retrospective analysis of the Michigan Spine Surgery Improvement Collaborative (MSSIC) registry, a prospective, multi-center, spine-specific database of patients surgically treated for degenerative disease. We determined causes for readmission and independent risk factors impacting readmission using multivariable logistic regression analysis.

Results: A total of 8533 patients who underwent lumbar fusion were included. Seven hundred sixty-three (8.9\%) patients were readmitted within the 90-day period. The most common specified reasons for readmission were surgical site infection (17\%), pain (16\%), and radicular symptoms $(10 \%)$. Risk factors associated with increased likelihood of readmission were diabetes (OR 1.54, CI 1.27-1.85), other race (OR 1.54, CI 1.01-2.24), multilevel fusions $>4$ levels, (OR 1.48, CI 1.14-1.92), surgery duration (OR 1.09, CI 1.04-1.15), and hospital length of stay (OR 1.01, CI 1.00-1.02). Factors associated with decreased risk of readmission were discharge to home (OR 0.71, CI 0.59-0.86), private insurance (OR 0.81, CI 0.68-0.96), and diagnosis of spondylolisthesis (OR 0.84, CI 0.72-0.98).

Discussion/Conclusion: After lumbar fusion surgery, 90-day readmission to the hospital occurs in approximately $9 \%$ of cases. Among many reasons for readmission, the most frequent reasons included wound infection, pain, and radicular symptoms. Among many factors impacting the likelihood of 90-day readmission, diabetes was the strongest risk factor, which presumably could be mitigated with strict preoperative and postoperative diabetic management. 
003. Lumbar Surgery within Two Weeks of Epidural Steroid Injection is Associated with Increased 90-Day Complications Jayme Koltsov, $P h D^{1}$, Matthew Smuck, $M D^{1}$, Todd Alamin, $M D^{2}$, Kirkham Wood, $M D^{2}$, Ivan Cheng, $M D^{3}$, Serena Hu, MD

\section{${ }^{1}$, Redwood City, California, ${ }^{2}$, Redwood City, CA, ${ }^{3}$ Stanford University Medical Center, Redwood City, CA}

Background/Introduction: ESIs given in close proximity to surgery may increase complication risk. The goal of this study was to use nationally-representative claims data to better understand the relationship between lumbar ESI and postoperative complications and the influence of the timing of ESI prior to surgery.

Materials/Methods: A primary lumbar spine surgery cohort was derived from the Marketscan® databases (2007-2014). Inclusion criteria were: surgical diagnosis of stenosis and/or disc herniation, age $\geq 18 \mathrm{yrs}$, health plan enrollment $\geq 1 \mathrm{yr}$ preoperatively and $\geq 90 \mathrm{~d}$ postoperatively to identify ESIs, screen exclusions, track complications. Patients with lumbar ESI prior to surgery were propensity score matched to those without ESI using all available demographics, comorbidities, and surgical details. Differences in 90-day complication rates were assessed with chi-squared tests, and the relationship between the timing surgery after ESI and complications was assessed via binary logistic regression with receiver operating characteristic (ROC) curves.

Results: Of patients having primary lumbar spine surgery for stenosis and/or herniation, 59.0\% had lumbar ESI within the prior year. 29,478 patients with ESI were matched successfully to those without ESI (age $50.2 \pm 14 \mathrm{yrs}, 41 \%$ female). No difference was observed in the overall rate of complications $(E S I=14.0 \%$, no_ESI $=14.2 \%$ p=0.530) or inpatient readmissions $(E S I=2.7 \%$, no_ESI=2.6\%, p=0.444). Additionally, infection rates did not differ between groups (ESI=2.0\%, no_ESI=1.9\%, $\mathrm{p}=0.475$ ). Despite no difference overall, complication rates increased as the time between ESI and surgery decreased (Figure). The optimal cut point was identified as 2 weeks. Patients having surgery within 2 weeks post-ESI had a $26 \%$ increased odds for complications $(\mathrm{OR}=1.26,95 \% \mathrm{CI}=1.11-1.42, \mathrm{p}<0.001)$ relative to those with more time before surgery. Drivers of this increase were identified through a sub-analysis comparing patients with "early surgery" following ESI $(n=3,082)$ and their matched pairs. Patients having surgery with 2 weeks of ESI had increased rates of infection (ESI=2.5\%, no_ESI=1.5\%,p<0.006), DVT (ESI=1.8\%, no_ESI=1\%, p=0.017), and inpatient readmissions $(\mathrm{ESI}=3.3 \%$, no_ESI $=2.3 \%, \mathrm{p}=0.017$ ) relative to those not having ESI prior to surgery.

Discussion/Conclusion: Lumbar ESI within 2 weeks prior to lumbar surgery for spinal stenosis or disc herniation is associated with increased risks of $66 \%$ for infection, $80 \%$ for DVT, and $43 \%$ for readmission within 90 days.

004. Spinal versus general anesthesia in lumbar spine surgery: a multicenter cost-effectiveness analysis

Matthew Morris, $B A^{1}$; Woojin cho, $M D, P h D^{2}$

${ }^{1}$, Bronx, New York, ${ }^{2}$ Montefiore Medical Center, Bronx, New York

Background/Introduction: Lumbar laminectomy may be performed under a variety of anesthetic techniques, but general anesthesia (GA) has traditionally been preferred. Several studies have demonstrated the safety and efficacy of spinal anesthesia (SA) as an alternative to GA for these procedures. However, a comparison of these two modalities in terms of both cost and clinical outcomes is necessary to determine their relative costeffectiveness.

Materials/Methods: Patients were grouped based on anesthetic modality. Perioperative data recorded included EBL, incidence of dural tear, anesthesia time, OR time, and surgical time. Postoperative data recorded included time in the PACU), pain scores, analgesic use, antiemetic use, incidence of nausea/vomiting, urinary retention, spinal headache, and 30-day readmission. Costs were calculated using variable hospital expenses directly related to patient care (direct costs).

Results: A total of 97 patients received SA and 91 received GA. The SA group spent less time in the OR $(138 \pm 5 \mathrm{vs.} 176 \pm 17 \mathrm{~min}, \mathrm{p}<0.05)$, PACU $(214 \pm 15$ vs. $249 \pm 27 \mathrm{~min}, \mathrm{p}<0.05)$, and under anesthesia $(149 \pm 5$ vs. $179 \pm 7 \mathrm{~min}, \mathrm{p}<0.05)$ than the GA group. The SA group also experienced less subjective postoperative pain $(0.64 / 10 \pm 0.38$ vs. $3.06 / 10 \pm 0.69, \mathrm{p}<0.05)$ and required less opioid analgesics $(1.13 \pm 0.36 \mathrm{vs}$. $4.30 \pm 0.87$ doses, $\mathrm{p}<0.05)$. Both groups experienced similar EBL and incidence of dural tears, nausea/vomiting, urinary retention, spinal headache, and 30-day readmission. Net operative cost at surgical site \#1 was found to be $\$ 8446.14 \pm 411.78$ with SA and $\$ 9285.78 \pm 509.57$ with GA (p<0.05), for a savings of $9.93 \%$. At site $\# 2$, net operative cost was found to be $\$ 9876.36 \pm 335.97$ with SA and $\$ 10362.59 \pm 802.59$ with GA, for a cost savings of $4.29 \%$ $(\mathrm{p}=0.286)$. A majority of this difference was attributable to greater OR time with GA.

Discussion/Conclusion: We conclude that use of SA for lumbar laminectomy leads to less OR, PACU, and anesthesia times, lower levels of postop pain, and no increased rate of other complications compared to GA. SA is 4.29-9.93\% less expensive than GA, indicating substantial costsaving potential. With no sacrifice of patient outcomes and the added benefit of less pain and recovery time, SA represents a more cost-effective alternative to GA in lumbar spine surgery.

005. The efficacy of liposomal bupivacaine in lumbar spine surgery

Emmett Gannon, $M D^{l}$, Chris Cornett, $M D^{2}$, Evan Larson, $M D^{3}$, Elizabeth Lyden, $M S$

${ }^{1}$ University of Nebraska Medical Center Department of Orthopaedic Surgery and Rehabilitation, OMAHA, NE, ${ }^{2} \mathrm{University}$ of Nebraska Medical Center Department of Orthopaedic Surgery and Rehabilitation, Omaha, NE, ${ }^{3}$ University of Nebraska Medical Center Department of Orthopaedic Surgery and Rehabilitation, Omah, NE, ${ }^{4}$ University of Nebraska Medical Center. Department of Biostatistics. College of Public Health, OMAHA, NE 
Background/Introduction: Pain management is an important portion of a patient's postoperative care. Improved pain control can lead to improved outcomes, earlier mobilization, and shortened hospital stays. With the large side-effect profile of narcotics, numerous alternative methods to decrease opioid consumption, attain better pain-control and improve outcomes have been used. The emergence of the long-acting local anesthetic agent, liposomal bupivacaine (LB), has shown promise in improving pain control and decreasing opioid consumption.

Materials/Methods: A total of 54 consecutive lumbar spine cases were performed in which LB was administered in all cases. This was then matched to a cohort of 54 patients who underwent lumbar procedures in the period immediately preceding the initial use of LB. Demographic data and hospital length of stay (LOS) were recorded. Pain scores were recorded using the visual analog scale (VAS) and were recorded as an average over 8 hour shifts for 72 hours postoperatively. Daily narcotic consumption was also measured and converted to oral morphine equivalents.

Results: There was a statistically significant difference found in the mean LOS between the two groups ( $\mathrm{p}=0.0071)$. The mean LOS for the LB group was 2.04 days and 2.73 days for the control group. A significant difference was also found between the two groups in VAS scores ( $\mathrm{p}=0.016$ ) and narcotic use $(\mathrm{p}=0.048)$. The LB group was found to have lower VAS scores and narcotic consumption compared to the control group.

Discussion/Conclusion: The use of LB shows promise as an adjuvant for postoperative analgesia in lumbar spine surgery by potentially decreasing pain scores, LOS, and narcotic use. The findings from this study would suggest that LB may be worth the increased cost, as being able to control a patient's pain can not only lead to improved outcomes, but also lead to cost savings by decreasing the LOS and consumption of narcotics. The recent findings from studies investigating the use of LB in spinal surgery, along with the results from this study demonstrate the need for further investigation, namely a larger prospective randomized control study.

006. Effect of perioperative medication and patient factors in postoperative urinary retention after elective spine surgery

Sapan Gandhi, $M D^{1}$, Jeff Cross, $B S^{2}$, Megan Hsu, $B S^{2}$, Christian Peterson, $B S^{2}$, Daniel Park, MD

\section{${ }^{l}$ Beaumont Health System, Royal Oak, MI, ${ }^{2}$ Oakland University William Beaumont School of Medicine, Royal Oak, MI, ${ }^{3}$, Royal Oak, MI}

Background/Introduction: POUR is a common postoperative complication after spine surgery, leading to increased length of stay, increased costs, and in some cases, long term genitourinary morbidity. Although some have described patient and surgical factors associated with POUR development after spine surgery, sparse literature exists regarding anesthetic and narcotic medication related to development of POUR after spine surgery.

Materials/Methods: After IRB approval, a retrospective review was conducted of all elective spine surgical cases performed by 2 spine surgeons at single institute from January 2016 through December 2016. Data collected included development of POUR, demographic variables (age, sex, body mass index), length of stay, comorbid conditions, mobilization within the first 24 hours after surgery, preoperative and postoperative narcotic use, perioperative medication administration, and surgical data.

Results: 709 elective spine cases were reviewed (193 cervical, 486 lumbar, and 30 thoracolumbar), with 186 cases of POUR (26.2\%). Development of POUR was associated advanced age (64.1vs56.8 years, $\mathrm{p}<0.001)$, higher number of levels (median) (2vs1, p=0.007), male sex (OR:1.92, p=0.0002), thoracolumbar cases ( $47 \%$ vs $25.9 \%$ cervical \& $25.1 \%$ lumbar, p=0.03), history of BPH (OR:3.30, p<0.0001), previous history of urinary retention (OR:10.42, $<<0.0001)$, use of GU medications at home (OR:4.36, $<<0.0001)$. Mobilization in the first 24 hours trended towards lower rates of POUR, but did not reach statistical significance (OR:0.56, $\mathrm{p}=0.07)$. Home narcotic use was not associated with POUR ( $\mathrm{p}=0.26)$. Lower intraoperative fentanyl dosage, as well as lower propofol dosage, was associated with the development of POUR (100vs $125 \mathrm{ug}$, p=0.01, 155vs190mg, $\mathrm{p}=0.03$, respectively). Those patients who received intraoperative IV hydromorphone were less likely to develop POUR (OR:0.63, $\mathrm{p}=0.01)$. Higher neostigmine dose was associated with development of POUR (median:5vs4mg, $\mathrm{p}=0.047$ ). There was no difference in postoperative morphine equivalent narcotic consumption between patients who developed POUR and those did not (115vs 102, $\mathrm{p}=0.50)$.

Discussion/Conclusion: Age, sex, number of levels, history of BPH, and history of retention were associated with development of POUR. Interestingly, higher intraoperative narcotic and propofol use was associated with lower rates of POUR, while higher dosage of neostigmine was associated with higher rates of POUR. Preoperative or postoperative narcotic utilization was not associated with POUR. Multivariate analysis is ongoing.

\section{Culturing patterns and results in presumed aseptic revision spine surgery}

Michael Steinhaus, $M D^{1}$, Stephan Salzmann, $M D^{2}$, Grant Shifflett, $M D^{3}$, Jingyan Yang, $M H S^{4}$, Janina Kueper, $B S^{5}$, Andrew Sama, $M D^{2}$, Federico Girardi, $M D^{6}$, Frank Cammisa, $M D^{2}$, Alexander Hughes, $M D$

${ }^{1}$ Hospital for Special Surgery, New York, NY, ${ }^{2}$ Hospital for Special Surgery, New York, New York, ${ }^{3}$ Rush University Medical Center, Chicago, IL, ${ }^{4}$ Hospital for Special Surgery, New York, New York, ${ }^{5}$, New York, New York, ${ }^{6}$, New york, New York

Background/Introduction: In the setting of negative or equivocal infectious workup prior to revision spine surgery, there is little guidance on when to consider intraoperative cultures to exclude concomitant infection. The purpose of this study was to report on culture collection patterns and results associated with revision spine cases at our institution. An improved understanding of the underlying characteristics which are associated with the decision to culture as well as the risk factors for positive cultures will help inform future culturing practices.

Materials/Methods: We retrospectively reviewed 595 consecutive revision spine surgeries performed by four senior spine surgeons between 2008-2013. Preoperative workup revealed the diagnosis of infection in 17 cases which were excluded from review. The remaining 578 presumed aseptic cases were included. Univariate and multivariate regression analyses were performed to determine variables associated with the decision to culture and risk factors for positive cultures. 
Results: Operative cultures were obtained in $112(19.4 \%)$ cases and were positive in 45/112 (40.2\%). The most common revision surgical diagnoses were recurrent index disease (43.8\%), pseudarthrosis $(22.0 \%)$, and adjacent segment disease (18.3\%). For cultured patients, pseudarthrosis was the most common revision diagnosis $(49.1 \%)$. Propionibacterium acnes was the most common organism isolated from positive cultures (48.8\%). Multivariate analysis demonstrated that obesity $(\mathrm{OR}=2.4)$, prior fusion $(\mathrm{OR}=2.4)$, time between index and revision $(\mathrm{OR}=0.9)$, thoracolumbar surgery $(\mathrm{OR}=5.2)$, cervical surgery $(\mathrm{OR}=0.2)$, and diagnosis of hardware failure $(\mathrm{OR}=9.0)$ or pseudarthrosis $(\mathrm{OR}=8.0)$ were significantly correlated with decision to culture, with AUC 0.85. Regarding culture results, multivariate analysis demonstrated that male sex $(\mathrm{OR}=3.4)$, fusion $(\mathrm{OR}=0.3)$, and revision diagnosis of pseudarthrosis $(\mathrm{OR}=4.1)$ were significantly associated with having positive cultures, with AUC 0.71 (Table 1).

Discussion/Conclusion: Unexpected positive cultures occurred in nearly half of cases and propionibacterium acnes was the predominant organism which speciated. Obesity, pseudarthrosis, prior fusion, thoracolumbar (and not cervical) surgery, and longer interval between index and revision surgeries predicted culture collection. Male sex, pseudarthrosis, and non-fusion cases predicted positive cultures. Considering these results, we recommend cultures be obtained in revision cases for pseudarthrosis, even in the setting of negative infectious work-up preoperatively.

008. Deep spinal infection eradicated by implants coated with antibiotic cement

Joseph Laratta, $M D^{l}$, Joseph Lombardi, $M D^{l}$, Jamal Shillingford, $M D^{1}$; Hemant Reddy, $B S^{2}$, Borys Gvozdyev, MD ${ }^{3}$, Yongjung Kim, MD

${ }^{1}$ The Spine Hospital at Columbia University, New York, NY, ${ }^{2}$ Northeast Ohio Medical University, Rootstown, OH, ${ }^{3}$ Norton Leatherman Spine Center, Louisville, $\mathrm{KY},{ }^{4}$, New York, $\mathrm{NY}$

Background/Introduction: Surgical site infection (SSI) is a devastating complication of spine surgery. Unlike the gold-standard two-stage revision in North American hip and knee arthroplasty, there exists no standardized, accepted protocol for the management of deep SSI with instrumentation. Because removal of hardware in an unstable, instrumented spine can result in serious neurologic sequelae, retention of instrumentation with elimination of bacterial colonization on implants is the goal.

Materials/Methods: Using CPT codes, institutional medical records were queried to identify all posterior spinal procedures performed by the senior surgeon from 2008 through 2014. Each record was reviewed on a case-by-case basis, and 34 patients were identified as having an implantassociated SSI. Exclusion criteria included: (1) superficial SSI (18 patients), (2) those not treated with the novel protocol (6 patients), and (3) those with less than 30 months of follow up. The study population consisted of 10 patients with deep implant-associated SSI who underwent our novel protocol of operative debridement and permanent coating of exposed implants with high-dose antibiotic cement.

Results: Clearance of infection was defined as absence of symptoms as outlined by CDC/NHSN criteria including: erythema, warmth, pain or tenderness, and purulent drainage among others. At two-year follow-up, none of the ten patients (0\%) in our series had evidence of continued deep infection and none required removal of hardware. Five of the ten patients $(50 \%)$ were able to clear infection with a single stage debridement and coating with antibiotic cement.

Discussion/Conclusion: Implant-associated SSI in the spine is potentially devastating. Prolonged hospitalization, long-term antibiotic therapy, management of secondary complications, and the need for repeated debridement procedures in the operating room results in significant morbidity to the patient and economic burden on the entire healthcare system. Herein, we describe a deep infection treatment protocol involving permanent implantation of antibiotic-loaded cement that is effective in preserving spinal instrumentation during infection eradication, preventing infection recurrence, and minimizing operative debridements.

\section{Visceral obesity on standard imaging predicts short-term survival in patients treated surgically for vertebral metastases}

Zach Pennington, B.S. ${ }^{1}$, A. Karim Ahmed, B.S. ${ }^{2}$, C. Rory Goodwin, MD, PhD ${ }^{3}$, Eric Sankey, M.D. ${ }^{4}$, Daniel Sciubba, MD ${ }^{5}$, Jorrit-Jan Verlaan, M.D., Ph.D. ${ }^{6}$, Bart Pielkenrood, Ph.D. ${ }^{7}$

${ }^{1}$ Johns Hopkins University, Baltimore, MD, ${ }^{2}$ Johns Hopkins School of Medicine, Baltimore, MD, ${ }^{3}$, Durham, NC, ${ }^{4}$ Johns Hopkins School of Medicine, Durham, NC, ${ }^{5}$ Johns Hopkins Hospital, Baltimore, Maryland, ${ }^{6}$ University Medical Center Utrecht, Utrecht, The Netherlands, ${ }^{7}$ University Medical Center Utrecht, Utrecht, Utrecht

Background/Introduction: In most patients operatively managed for vertebral metastases, the goal of surgery is palliation rather than cure. Even so, expected patient survival heavily influences the decision to operate. We seek to demonstrate that pre-operative body fat and muscle volumes as assessed on standard-of-care CT imaging are independent predictors of post-operative survival in patients with vertebral metastases.

Materials/Methods: We reviewed all patients treated at Johns Hopkins for vertebral metastases between 8/1/2004-7/1/2013 with pre-operative abdominal CT volumes acquired less than 3 months prior to surgery. Data were collected on pre-operative neurologic status, non-operative adjuvant treatments, body composition, health comorbidities, extent and pathology of oncologic disease, and Tomita and Tokuhashi scores. Stepwise multivariable logistic regressions were then used to determine independent predictors of $3 \mathrm{mo}, 6 \mathrm{mo}$, and $12 \mathrm{mo}$ survival.

Results: We included 99 patients (median age 59,59.6\% male, $65.7 \%$ white, 30.3\% black) with the most common primary lesions being lung $(16.2 \%)$, prostate $(14.1 \%)$, kidney $(11.1 \%)$, and breast (10.1\%). Independent predictors of 3mo survival were post-operative chemotherapy (95\% CI: 1.01-3.41; $\mathrm{p}=0.04)$, visceral fat area $(95 \% \mathrm{CI}: 1.00-1.18$ per $1000 \mathrm{~mm} 2 ; \mathrm{p}=0.04)$, and a Tomita score $<3(95 \% \mathrm{CI}: 1.17-19.93 ; \mathrm{p}=0.03)$. The only independent predictor of survival at $6 \mathrm{mo}$ was VFA (95\% CI: $1.01-1.21$ per $1000 \mathrm{~mm} 2 ; \mathrm{p}=0.04)$. Independent predictors of $12 \mathrm{mo}$ survival were breast primary pathology (95\% CI: $1.17-18.81 ; \mathrm{p}=0.03)$, an absence of visceral metastases $(95 \% \mathrm{CI}: 1.25-4.36$; $\mathrm{p}=0.008)$, being ambulatory preoperatively (95\% CI: $1.07-4.95 ; \mathrm{p}=0.03)$, and subcutaneous fat area $(95 \% \mathrm{CI}: 1.00-1.07$ per $1000 \mathrm{~mm} 2 ; \mathrm{p}=0.05)$. 
Discussion/Conclusion: Visceral fat mass was an independent, positive predictor of short-term post-operative survival in patients treated for vertebral metastases. As a result, we believe that the prognostic accuracy of current prognostic tools may be improved by the addition of visceral fat volume as a risk factor.

\section{Implementation of a non-operative care pathway for stable thoracolumbar fractures}

Adam Pearson, $M D, M S^{1}$; Gregory Hanson, $M P H^{2}$, William Abdu, $M D, M S^{3}$

\section{${ }^{1}$, Lebanon, $\mathrm{NH},{ }^{2}$ Geisel School of Medicine at Dartmouth, Lebanon, NH, ${ }^{3}$ Dartmouth-Hitchcock Medical Center, Lebanon, NH 03766, NH}

Background/Introduction: There is marked variation in the treatment of stable TL fractures. A standardized care pathway for these injuries could potentially reduce cost and LOS.

Materials/Methods: A multidisciplinary team at a Level 1 trauma center reviewed care processes for the non-operative treatment of stable TL fractures and identified marked variation in the rate and types of bracing, number of radiographs ordered, and length of stay. A new care pathway was created and embedded within the electronic medical record. We analyzed the rate and type of bracing, number of thoracolumbar radiographs following fracture diagnosis, cost of inpatient care, and length of stay (LOS) before and after pathway adoption. Differences between the neurosurgery and orthopaedic spine service were also compared.

Results: Of the 406 non-operative TL fracture patients, 183 (45.1\%) were braced (60.6\% custom-made TLSO, 39.4\% off the shelf TLSO). Fracture radiographs were significantly reduced after initiation of the care pathway (3.23 vs $2.63, \mathrm{p}=.010)$. The total cost for braced patients after pathway adoption trended lower $(\$ 8928.58$ vs. $\$ 10,462.36, \mathrm{p}=.078)$, primarily due to lower bracing costs $(\$ 1352.41 \mathrm{vs} . \$ 3719.53, \mathrm{p}<.001)$. The mean LOS and complication rate did not change significantly following pathway adoption. The orthopaedic spine service braced less frequently than the neurosurgery service overall $(40.7 \%$ vs. $52.2 \%, \mathrm{p}=.023)$, with both teams adopting the less expensive off the shelf TLSO over time.

Discussion/Conclusion: The use of a care pathway that helps to standardize bracing and radiography for stable TL fracture patients can reduce cost and radiation exposure without increasing complication rates.

011. A novel quantitative computed tomography bone mineral density assessment of the sacrum does not predict sacral fractures following instrumented spinal fusion

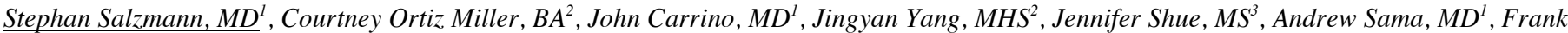
Cammisa, $M D^{l}$, Federico Girardi, $M D^{4}$, Alexander Hughes, $M D^{l}$

${ }^{l}$ Hospital for Special Surgery, New York, New York, ${ }^{2}$ Hospital for Special Surgery, New York, New York, ${ }^{3}$ Hospital for Special Surgery, New York, New York , ${ }^{4}$, New york, New York

Background/Introduction: Postoperative sacral fractures are a rare but potentially devastating complication. Long-fusion constructs including the sacrum that do not extend to the pelvis may result in sacral fractures. Osteoporosis has been proposed as an important risk factor, however, data supporting this is primarily anecdotal. The objective of this study was to evaluate the association between preoperative bone mineral density (BMD) of the sacrum and the occurrence of sacral fractures following lumbar instrumented spinal fusion.

Materials/Methods: Patients with sacral fractures following posterior instrumented fusion including the sacrum were retrospectively identified and matched 2:1 with nonfracture controls based on sex, age and number of levels fused. Patients with concurrent spinopelvic fixation or missing preoperative CT imaging were excluded. Standard quantitative computed tomography (QCT) measurements at L1 and L2 and additional experimental measurements of the sacrum, including the $\mathrm{S} 1$ body and the sacral ala, were performed.

Results: Twenty-one sacral fracture patients had preoperative CT imaging and were matched to nonfracture controls. The groups were well matched with respect to sex, age and number of levels fused. Standard measurements at L1/L2 showed no significant difference between the fracture and the control group $(109.9 \mathrm{mg} / \mathrm{cm} 3 \mathrm{vs} .116 .4 \mathrm{mg} / \mathrm{cm} 3, \mathrm{p}=0.414)$. Similarly, there was no significant BMD differences between the fracture and control group using the experimental measurements of the $\mathrm{S} 1$ body $(183.6 \mathrm{mg} / \mathrm{cm} 3 \mathrm{vs} .176 .2 \mathrm{mg} / \mathrm{cm} 3, \mathrm{p}=0.567)$ and the sacral ala $(8.9 \mathrm{mg} / \mathrm{cm} 3 \mathrm{vs}$. $4.8 \mathrm{mg} / \mathrm{cm} 3, \mathrm{p}=0.616$ ). However, univariate logistic regression revealed that the odds of experiencing a sacral fracture was approximately 6 times higher for obese patients compared to normal or underweight patients. After controlling for BMI in multivariate conditional logistic regression models, BMD was still not significantly associated with the odds of experiencing sacral fractures.

Discussion/Conclusion: There was no association of preoperative BMD and the occurrence of sacral fractures. Obesity, however, was associated with higher odds of experiencing postoperative sacral fractures. This is in line with previous reports that indicate only a few sacral fracture patients after fusion surgery have clear evidence of osteoporosis. BMD as a measure of bone quantity, rather than bone quality, may not be as important in these fractures as previously thought.

\section{Does Lumbar Spine Pathology Affect Bone Mineral Density Measurement by Quantitative Computed Tomography (QCT)?}

Stephan Salzmann, $M D^{l}$, Brandon Carlson, $M D, M P H^{l}$, Toshiyuki Shirahata, $M D^{1}$, Courtney Ortiz Miller, BA ${ }^{2}$, John Carrino, $M D^{l}$, Jingyan Yang, $M H S^{2}$, Andrew Sama, $M D^{l}$, Frank Cammisa, $M D^{1}$, Federico Girardi, MD ${ }^{3}$, Alexander Hughes, $M D^{l}$

${ }^{l}$ Hospital for Special Surgery, New York, New York, ${ }^{2}$ Hospital for Special Surgery, New York, New York, ${ }^{3}$, New york, New York 
Background/Introduction: Lumbar degenerative changes can lead to an overestimation of bone mineral density (BMD) by dual-energy x-ray absorptiometry (DXA). Similarly, quantitative computed tomography (QCT) can be affected by degenerative/sclerotic vertebral body changes if inadvertently included during the measurement process. It remains unclear if excluding pathologic bone regions in the selected field allows for accurate BMD measurements. The aim of this study was to assess whether BMD measured from pathologic levels is different than BMD derived from non-pathologic levels in paired, matched controls.

Materials/Methods: Patients undergoing posterior lumbar fusion from 2014 to 2017 with available preoperative CT imaging were included. QCT measurements of L1-S1 were performed. Pathologic levels were defined by referencing surgical procedure records. Degenerative/sclerotic bone regions at pathologic levels were avoided during the measurement process to minimize overestimation of BMD (Figure 1). Due to known BMD differences at different lumbosacral levels, comparisons between pathologic and normal levels within the same patient are not possible. To overcome this limitation, matched normal-pathologic measurements at the same lumbosacral level were identified in matched patient-pairs. Patients were matched 1:1 based on gender, age (+/-5yrs), BMI (+/-5), and race. One or more normal-pathologic matched levels were identified from each patient pair. Differences between normal and pathologic levels were assessed using two-tailed paired t-tests with significance set at $\mathrm{P}<0.05$.

Results: Seventy-seven matched patient pairs (154 patients) were isolated from a cohort of 296 patients. Patients not matched were excluded due to inability to match on all four matching criteria. From the 77 patient pairs, 152 matched normal-pathologic levels were identified for analysis. The mean (SD) for normal and pathologic levels was $140.4 \mathrm{mg} / \mathrm{cm} 3(54.13)$ and $143.0 \mathrm{mg} / \mathrm{cm} 3$ (49.04), respectively. Paired statistical analyses revealed no significant difference between groups $(\mathrm{P}=0.563)$.

Discussion/Conclusion: Our data demonstrates no difference in BMD measured at pathologic and non-pathologic vertebra at the same lumbar/sacral levels in paired, matched controls. When using the recommended methodology for QCT measurements including the avoidance of degenerative/sclerotic regions, BMD can be accurately measured at pathologic levels. Our findings suggest that QCT measurements, in contrast to DXA, may not be affected by degenerative changes and may more accurately represent lumbar BMD.

\title{
013. The Utility of Intraoperative Neuromonitoring for Minimally Invasive Lateral Approach to the Lumbar Spine
}

Miranda Bice, $M D^{1}$; James Stokman, $M D^{2}$, Nathaniel Wilson, $M D^{l}$, Scott Vincent, $M D^{3}$, Seth Williams, $M D^{4}$

\author{
${ }^{1}$ UNIVERSITY OF WISCONSIN, Madison, WI, ${ }^{2}$, Madison, WI, ${ }^{3}$ University of Nebraska Medical Center, Omaha, NE, ${ }^{4}$ University of \\ Wisconsin, Madison, WI
}

Background/Introduction: Lateral interbody fusion for the lumbar spine is a surgical procedure that can address multiple pathologies a minimally invasive (MIS) manner. Because of the concern for iatrogenic neurological injury, intraoperative neuromonitoring (IONM) utilization is advocated to theoretically decrease this risk. Supporters do acknowledge that IONM does not eliminate this risk. We hypothesize that, with rare exception, IONM for the MIS lateral approach to the spine does not change intraoperative decision making, particularly in the upper lumbar spine (T12-L2). The purpose of this study was to determine frequency and associations of IONM responses for this approach.

Materials/Methods: A retrospective chart review was performed. The primary outcome was IONM response. This was classified as "no" if there were no events on IONM, and "yes" if there was an event on IONM which resulted in retractor repositioning or discontinuation of the procedure at the planned level. Secondary outcomes included associations of IONM responses to surgical level, psoas size, comorbidities, and new postoperative symptoms.

Results: Sixty-three patients with 87 levels were included. There were five "yes" IONM responses (5.7\%). In the upper lumbar spine, there were no positive responses. Frequency of response increased with each caudal level (figure 1). New approach-side leg pain occurred in $20 \%$ of patients, regardless of IONM response.

Discussion/Conclusion: Reports of new postoperative neurologic complications for MIS lateral interbody fusion vary widely, even with routine use of IONM. Intraoperative neuromonitoring incurs a cost, and may not be necessary or beneficial in all patients. In the present study, there were no positive responses in the upper lumbar spine, and the frequency of positive responses increased with caudal levels. Female sex, greater BMI, presence of diabetes, and greater psoas AP dimension were factors associated with positive responses. The frequency of new postoperative leg symptoms was the same for both "yes" and "no" responders. These results suggest that detection of a response may be predictable, but even if present, may not change outcome. Considering this information, perhaps risk stratification, rather than uniform utilization of IONM, will decrease incidence of postoperative neurological complications associated with the lateral MIS approach to the lumbar spine.

\section{The duration of lumbar radicular symptoms does not impact clinical outcomes following lumbar decompression surgery}

Bryce Basques, $M D^{1}$, Philip Louie, $M D^{1}$; Michael Nolte, $M D^{2}$, Victor Lei, BS $S^{3}$, Jeremy Mormol, BS ${ }^{1}$, Arya Varthi, MD ${ }^{4}$, Justin Paul, $M D$, PhD , Edward Goldberg, $M D^{6}$, Howard An, $M D^{l}$

${ }^{1}$ Rush University Medical Center, Chicago, IL, ${ }^{2}$ Rush University Medical Center, Chicago, Illinois, ${ }^{3}$, Chicago, Illinois, ${ }^{4}$ Yale University , New Haven, CT, ${ }^{5}$, New York, NY, ${ }^{6}$, Chicago, IL

Background/Introduction: The success of surgical interventions for lumbar spinal stenosis can vary significantly depending on various factors, one of which is the duration of symptoms. We sought to assess whether the duration of symptoms has an effect on clinical outcomes, especially resolution of radicular symptoms in patients undergoing lumbar decompression-only procedures.

Materials/Methods: We performed a retrospective cohort analysis of patients who underwent a primary lumbar laminectomy, discectomy, or a combination of the two. Patient reported outcomes were obtained in the form of Oswestry Disability Index (ODI) scores, Visual Analog Scales 
(VAS) scores for the back and leg, 12-Item Short Form Mental and Physical Survey (SF-12) scores, and the Veterans Rand 12-Item Health Mental and Physical Survey (VR-12) scores. Patients were also surveyed about their expectations and satisfactions following surgery.

Results: Overall, 210 consecutive patients with average follow-up was 24.14 months (range 6-78 months), fulfilled inclusion criteria. There were 108 patients that experienced radicular symptoms for less than one year, while 102 patients experienced radicular symptoms for one year or more. Patients who experienced radicular symptoms for less than one year had a significantly higher rate of a herniated nucleus pulposus (HNP) present ( $75.9 \%$ vs $49.0 \%$; $<0.001)$. No other significant differences in the demographic variables between the 2 groups. Patients with symptoms for one year or greater presented with significantly lower SF-12 physical scores $(32.0 \pm 11.1$ vs $54.7 \pm 8.3 ; \mathrm{p}=0.043)$ compared to those with symptoms for less than one year. No other significant differences in other outcome survey scores pre- and post-operatively. Re-operation rates were not significantly different. Patients presented with high levels of satisfaction (pain $<1$ year $91.7 \%$ vs. pain $\geq 1$ year $82.4 \%$; OR 1.06 , p=0.904) and expressed that the surgery met or exceeded their expectations (pain<1 year 100\% vs. pain $\geq 1$ year $96.1 \%$; OR1.06, p=0.904).

Discussion/Conclusion: The duration of radicular symptoms, in patients with lumbar spinal stenosis and/or a herniated nucleus pulposus, does not impact their clinical outcomes 2 years after lumbar decompression surgery. Re-operation rates were low and similar between the 2 groups of patients. Most patients report being satisfied with their outcome and with their expectations being met or exceeded to similar degrees.

\section{The effect of preoperative symptom duration on postoperative outcomes following a tubular lumbar microdiscectomy}

Bryce Basques, $M D^{l}$; Brittany Haws, $B S^{2}$, Benjamin Khechen, $B A^{2}$, Philip Louie, $M D^{l}$, Kamran Movassaghi, $M S^{2}$, Kaitlyn Cardinal, BS ${ }^{2}$, Jordan Guntin, $B S^{2}$, Kern Singh, $M D^{1}$

\section{${ }^{1}$ Rush University Medical Center, Chicago, IL, ${ }^{2}$ Rush University Medical Center, Chicago, Illinois}

Background/Introduction: There is considerable debate regarding the appropriate duration of nonoperative treatment before surgery is considered for a lumbar disk herniation. It is unknown if extended nonoperative treatment has implications for long term clinical outcomes even after surgery is performed. This study aims to characterize the effect of preoperative symptom duration on postoperative outcomes following minimally invasive lumbar microdiscectomy (MIS LD).

Materials/Methods: Patients undergoing MIS LD were reviewed. Preoperative symptom duration was dichotomized into two groups ( $\leq 6$ months, $>6$ months). Only patients with full clinical data at 6 months postoperative follow-up were included in the study. Clinical outcomes including Oswestry Disability Index (ODI), Visual Analog Scale (VAS) Back, and VAS Leg were assessed at 6-weeks, 12-weeks, and 6-months postoperative. The amounts of patients obtaining a minimum clinically important difference (MCID) for each clinical outcome was also assessed. Groups were compared with chi-square analysis and student t-tests for categorical and continuous data, respectively.

Results: 94 patients were identified, 45 with symptom duration $\leq 6$ months and 49 with symptom duration $>6$ months. No differences in baseline or perioperative characteristics were found $(\mathrm{p}>0.05)$. When comparing patient reported outcomes, patients with shorter symptom duration had significantly greater improvement in ODI scores at 6 weeks $(p=0.004), 12$ weeks $(p=0.022)$, and 6 months $(p=0.005)$ was found for patients with shorter preoperative symptom duration. Patients with shorter symptom duration also obtained MCID for ODI at a significantly greater rate than those with longer duration of symptoms $(\mathrm{p}=0.015)$. Additionally, patients with a shorter duration of symptoms had significantly greater improvement in VAS leg pain at 6-months, though this was not statistically significant. For VAS back pain, no differences were found between groups.

Discussion/Conclusion: Patients that underwent MIS LD within 6 months of symptom onset had similar baseline characteristics compared to patients who underwent surgery after 6 months. However, the patients with shorter preoperative symptom duration had greater clinical improvement postoperatively. Further studies should evaluate the efficacy of non-operative treatment in the setting of lumbar disk herniations particularly as it may potentially impair functional recovering following delayed surgical intervention.

016. Anterior lumbar, oblique lateral, and transforaminal lumbar interbody fusions: a comparison of perioperative complications

Caleb Edwards, $B A^{l}$, Anthony DiGiorgio, DO, $M H A^{2}$, Darryl Lau, $M D^{3}$, Praveen Mummaneni, $M D^{4}$; Dean ${\text { Chou, } M D^{3}}^{3}$

${ }^{l}$ University of California-San Francisco School of Medicine, San Francisco, CA, ${ }^{2} \mathrm{LSU}$ Health Sciences Center, New Orleans, LA, ${ }^{3}$ University of California, San Francisco, San Francisco, California, ${ }^{4}$, San Francisco, California

Background/Introduction: Interbody fusions can be performed via anterior lumbar (ALIF), oblique lateral (OLIF), and transforaminal (TLIF) approaches. We sought to compare complication rates of these modalities.

Materials/Methods: Retrospective review of ALIF, OLIF, and TLIF cohorts was undertaken. Demographics, ASA scores, operative time, estimated blood loss, infection, intraoperative CSF Leak, vascular injury, post-operative ileus, motor deficits and 90-day readmissions were evaluated.

Results: 213 patients were included: 90 ALIF patients, 49 OLIF patients, 74 TLIF patients. OLIF patients were older than ALIF and TLIF (67y vs $61 \mathrm{y}$ and $61 \mathrm{y} ; \mathrm{p}<0.05$ ). ALIF cohort (52\% male; $67 \%$ prior surgery) had larger percentage of males and prior spinal surgeries than OLIF (37\%; $59 \%)$ and TLIF $(36 \% ; 54 \%)$. No significant differences were observed in ASA scores and procedure time. OLIF cases had significantly less blood loss compared to ALIF and TLIF $(229 \mathrm{ml}$ vs $814 \mathrm{ml}$ and $578 \mathrm{ml}$; $\mathrm{p}<0.05)$. Near-identical infection rates were seen for ALIF, TLIF, and OLIF (4\% vs $3 \%$ vs $2 \%$; p $>0.05$ ). TLIF had highest risk for durotomy (3\%), followed by ALIF (1\%) and OLIF (0\%). Vascular injury was highest for ALIF (8\%) compared to OLIF (2\%) and TLIF (0\%). Post-operative ileus was highest in ALIF patients (20\%), compared to OLIF (6\%) and TLIF (3\%). At most recent follow-up, ALIF (19\%) and TLIF (15\%) had more motor deficits than OLIF (6\%). OLIF (4\%) had marginally lower rates of 90 -day surgery related readmissions than TLIF $(8 \%)$ or ALIF (7\%). 
Discussion/Conclusion: Within our series, OLIF had the lowest complication rate when compared to ALIF and TLIF. This may be because of the minimally invasive approach, avoidance of direct neural manipulation either posteriorly or through the lumbar plexus, and operating through a corridor without needing to mobilize the great vessels.

017. Patient Reported Outcomes Measurement Information System (PROMIS) instruments outperform traditional quality of life measures in patients with back and neck pain

Dennis Vasquez-Montes, $M S^{1}$, Jared Tishelman, $B A^{2}$, David S. Jevotovsky, BA ${ }^{2}$, Raj Karia, $M S^{2}$, Charla Fischer, $M D^{3}$, Aaron Buckland, $M D^{4}$, Thomas Errico, $M D^{2}$, Themistocles Protopsaltis, $M D$

\section{${ }^{1}$, New York, NY, ${ }^{2}$ Department of Orthopedic Surgery, NYU Langone Orthopedic Hospital, NYU Langone Health, New York, New York, ${ }^{3}$ Columbia University Department of Orthopaedic Surgery, New York City, New York, ${ }^{4}$ Department of Orthopedic Surgery, NYU Langone Orthopedic Hospital, NYU Langone Health, Manhattan, NY}

Background/Introduction: The Patient Reported Outcomes Measurement Information System (PROMIS) Physical Function, PROMIS Pain Intensity, and PROMIS Pain Interference are three instruments that have become popular for computer adaptive testing methodology. This study aims to validate the association between PROMIS and outcome measurements ODI, NDI and VAS for patients with back and neck pain.

Materials/Methods: A retrospective review of a PROM database was performed at a single institution from 12/2016 to 4/2017. Inclusion criteria were age $>18$ and a complaint of neck or back pain. PROMIS Pain Interference, Physical Function, and Pain Intensity were compared with ODI, NDI, VASBack, VASLeg, VASNeck, and VASArm. The relationship between PROMIS and traditional PROMs was assessed by neck and back pain. The number of questions was used to estimate the efficiency of the survey. Means were compared for significance at $\alpha=0.05$. Sensitivity was assessed by comparing the distribution in the lower/higher bounds of possible scores.

Results: 494 patients with back pain and 130 patients with neck pain were included for analysis. For back pain, ODI correlated all PROMIS instruments $(\mathrm{R}=-0.749, \mathrm{p}<0.001, \mathrm{R}=0.790, \mathrm{p}<0.001, \mathrm{R}=0.709, \mathrm{p}<0.001)$. On average, patients answered significantly fewer questions using the PROMIS Physical Function domain than ODI (4.123vs.9.906, $\mathrm{p}<0.001)$. When assessing for sensitivity, neither survey presented a significant Ceiling/Floor effect in the Back population (ODI: 0.40\%vs2.63\%, PROMIS PF: 0.60\%vs1.41\%). Similarly, for neck pain, NDI showed a strong correlation to all three PROMIS dimensions $(\mathrm{R}=-0.771, \mathrm{R}=0.786, \mathrm{R}=0.605$, all $\mathrm{p}<0.001)$. PROMIS Pain Intensity correlated to VASNeck ( $\mathrm{R}=0.642$, $\mathrm{p}<0.001)$, and mildly correlated with Arm Pain $(\mathrm{R}=0.376, \mathrm{p}<0.001)$. In comparing the mean number of questions answered, patients answered fewer questions for PROMIS than NDI $(4.417 \mathrm{vs} 10, \mathrm{p}<0.001)$. No significant differences were found in terms of Ceiling/Floor effects for patients with Neck complaints (NDI:0.00\%vs6.92\%, PF:0.00\%vs5.34\%).

Discussion/Conclusion: PROMIS dimensions correlate with traditional metrics in patients with back and neck pain. For both groups, the PROMIS PF questionnaire required fewer questions for similar granularity. There were no significant differences in ceiling/floor effects for NDI or ODI when compared with PROMIS PF. PROMIS domains are capable of detecting disability in neck/back pain with a more efficient questionnaire.

\section{Association Between Non-Modifiable Demographic Factors and Press Ganey Satisfaction Scores in Spine Surgery Clinics}

$\underline{\text { Bradley Johnson, } M D^{1}}$, Dennis Vasquez-Montes, $M S^{2}$, Aaron Buckland, $M D^{3}$, John Bendo, $M D^{4}$, Jeffrey Goldstein, MD ${ }^{5}$, Charla Fischer, $M D^{6}$

${ }^{1}$ New York University Langone Medical Center, New York, New York, ${ }^{2}$, New York, NY, ${ }^{3}$ Department of Orthopedic Surgery, NYU Langone Orthopedic Hospital, NYU Langone Health, Manhattan, NY, ${ }^{4}$ New York University Langone Medical Center, New York, CA, ${ }^{5}$ New York University Langone Medical Center, New York, NY, ${ }^{6}$ Columbia University Department of Orthopaedic Surgery, New York City, New York

Background/Introduction: Patient satisfaction is rapidly becoming an important metric in value-based care systems. Satisfaction is linked to both health-care quality assessment and compensation within the Affordable Care Act. The Press Ganey Associates satisfaction survey is the most widely used instrument for measuring patient satisfaction. Understanding the factors that influence these surveys may help to better utilize survey results and allow interventions to increase patient satisfaction.

Materials/Methods: Press Ganey Associates satisfaction surveys administered to ambulatory spine surgery clinic patients within a large tertiary care network from 05/2016 to 09/2017 were retrospectively reviewed for patient demographics and visit characteristics. Mean comparison testing was performed to compare demographic factors with responses to "overall provider rating" and "recommend this provider office" survey questions with the mean difference to achieve significance set at $\alpha<0.05$. A multivariate analysis was performed to determine independent factors.

Results: 1,400 survey responses from the offices of 11 fellowship trained orthopedic spine surgeons were included. Patients aged 18 to 34 had significantly lower responses to the "overall doctor rating" question than older patients $(\mathrm{p}<0.001)$, and increasing patient age was correlated with improving ratings. Highest education level was inversely correlated with satisfaction scores, with patients who attained graduate level education giving the lowest scores $(\mathrm{p}=0.001)$. Those with commercial insurance had significantly lower ratings to "recommend this provider" ( $\mathrm{p}=0.042)$ and "overall doctor rating" ( $p=0.022)$ than those with Medicare, Medicaid, worker's compensation insurance or no insurance. Patients administered the survey on paper had significantly lower ratings than those administered the survey online $(\mathrm{p}=0.006)$. Doctor ratings were significantly higher when the gender and ethnicity of the patient matched the gender and ethnicity of the provider $(\mathrm{p}=0.021)$.

Discussion/Conclusion: This study identifies several demographic factors which influence survey results. Importantly, it demonstrates that independent, non-modifiable factors like age, education level, survey mode, and insurance type influence satisfaction. It also suggests that patients may be more satisfied when they align with the gender and ethnicity of the provider. With a large enough sample size, it may be possible to create an algorithm to adjust for the influence of non-modifiable demographic factors. 
019. Defining the minimum clinically important difference for Grade I degenerative lumbar spondylolisthesis: insights from the Quality Outcomes Database M.D.

Panagiotis Kerezoudis, M.D. ${ }^{1}$, Andrew Chan, $M D^{2}$, Praveen Mummaneni, $M D^{3}$, Christopher Shaffrey, $M D^{4}, P^{2} a u l$ Park, $M D^{5}, M o h a m a d ~ B y d o n$,

\author{
${ }^{l}$ Mayo Clinic, Rochester, MN, ${ }^{2}$ Department of Neurological Surgery, University of California, San Francisco, San Francisco, CA, ${ }^{3}$, San
} Francisco, California, ${ }^{4}$ University of Virginia Medical Center, Charlottesville, VA, ${ }^{5}$, Ann Arbor, Michigan, ${ }^{6}$, Rochester, MN

Background/Introduction: The concept of minimum clinically important difference (MCID) is considered the new standard for determining the effectiveness of a given treatment and describing patient satisfaction in response to that treatment. Herein, we sought to determine the MCID associated with surgical treatment for degenerative lumbar spondylolisthesis.

Materials/Methods: We queried the Quality Outcomes Database registry from July 2014 through December 2015 for patients undergoing posterior lumbar surgery for grade I degenerative spondylolisthesis. Recorded patient reported outcomes included Oswestry Disability Index (ODI), EQ-5D, numeric rating scale (NRS)-leg pain and -back pain. Anchor-based (using the NASS satisfaction scale) and distribution-based (half a standard deviation, small Cohen's effect size, standard error of measurement and minimum detectable change (MDC)) methods were used to calculate the MCID for each PRO.

Results: A total of 441 patients (80 laminectomies alone, 361 fusions) from 11 participating sites were included in the analysis. Change in functional outcomes scores between baseline and 1-year were $23.5 \pm 17.4$ points for ODI, $0.24 \pm 0.23$ for EQ-5D, $4.1 \pm 3.5$ for NRS-LP, and 3.7 \pm 3.2 for NRS-BP. The different calculation methods generated a range of MCID values for each PRO: 3.3 to 26.5 points for ODI, 0.04 to 0.3 points for EQ-5D, 0.6 to 4.5 points for NRS-leg pain and 0.5 to 4.2 points for NRS-back pain. The MDC approach appeared to be the most appropriate for calculating MCID because it provided a threshold greater than the measurement error and was closest to the average change difference between the satisfied and not satisfied patients. On subgroup analysis, the MCID thresholds for laminectomy alone patients were comparable to those undergoing arthrodesis as well as the entire cohort.

Discussion/Conclusion: The MCID for PROs was highly variable depending on calculation technique. The MDC seems to be a statistically and clinically sound method for defining the appropriate MCID value for patients with grade I degenerative lumbar spondylolisthesis. Based on this method, the MCID values are 14.3 points for ODI, 0.2 points for EQ-5D, 1.7 points for leg pain, and 1.6 points for back pain.

\title{
020. Patient profiling can identify spondylolisthesis patients at risk for conversion from nonoperative to surgical treatment
}

Peter Passias, $M D^{1}$, Gregory Poorman, $B A^{2}$, Samantha Horn, $B A^{1}$, Cole Bortz, $B A^{1}$, Frank Segreto, BS ${ }^{1}$, Shay Bess, MD ${ }^{3}$, Virginie Lafage, PhD ${ }^{4}$, Michael Gerling, $M D^{5}$, Thomas Errico, $M D$

\footnotetext{
${ }^{1}$, New York, NY ${ }^{2}$, bronxville, ny, ${ }^{3}$, Denver, Colorado, ${ }^{4}$ Hospital for Special Surgery, New York, NY, ${ }^{5}$, TriBeCa, NY, ${ }^{6}$ Department of Orthopedic Surgery, NYU Langone Orthopedic Hospital, NYU Langone Health, New York, New York
}

Background/Introduction: Factors relevant in deciding surgical treatment for degenerative spondylolisthesis (DS) include disease state severity and patient quality of life expectations. Some may not be easily appraised by the surgeon. In a prospective trial with nonoperative and operative patients, there are crossovers from the nonoperative group who receive surgery. Identifying and understanding patient characteristics that may influence crossover from nonoperative to operative treatment arms will aid understanding of what motivates patients towards pursuing surgery.

Materials/Methods: DS patients randomized to nonoperative care in a prospective, multicenter study were evaluated over 8 years of enrollment. Two cohorts were defined: (1) crossover (CROSS); those who at any point received surgery, and non-crossover (non-CROSS); those who remained nonoperative. A Cox proportional hazards model, modeling time to crossover, was used to explore demographic data, clinical diagnoses, and patient expectations and attitudes after adjusting other variables. A sub-analysis was performed on crossovers within 6 months of enrollment and crossovers after 6 months of enrollment.

Results: 145 randomized nonoperative patients were included, 80 of which crossed over to surgery. In analyzing baseline differences, CROSS patients were younger however there was no significant difference in race, sex, or comorbidities. Without considering treatment preference, Cox proportional hazards modeling described younger age, male sex, and white-race as predictors of crossover. Upon inclusion of treatment preference as a factor, treatment preference and 'problem getting worse' were the only predictors for crossover. Clinically, stenosis, neurological deficits, and listhesis levels did not show a significant relationship with crossing over. At long-term followup, CROSS showed significantly greater long-term HRQL improvement. The difference was maintained throughout follow-up.

Discussion/Conclusion: Patient reported outcome scores, neurological symptoms, and diagnoses including listhesis and stenosis severity did not predict patients randomized to nonoperative care crossing over to surgery. Self-evaluation of symptoms and attitudes towards surgery were the only independent predictors of crossover from nonoperative to operative care. Certain demographics crossed over at higher rates, but not independently from attitudes towards surgery.

021. Higher improvement in patient reported outcomes can be achieved after transforaminal lumbar interbody fusion for CARDS Type D degenerative lumbar spondylolisthesis

Xu Sun, M.D. ${ }^{1}$, Xi Chen, $M D^{2}$, Zezhang Zhu, M.D. ${ }^{3}$, Yong Qiu, M.D. ${ }^{2}$, Zhen Liu, M.D. ${ }^{4}$ 
${ }^{1}$ Spine Surgery, Drum Tower Hospital, Nanjing University Medical School, Nanjing, Jiangsu, ${ }^{2}$ Spine Surgery, Drum Tower Hospital, Nanjing University Medical School, Nanjing, Jiangsu, ${ }^{3}$, Nanjing, Jiangsu, ${ }^{4}$, Nanjing, Jiangse

Background/Introduction: The newly proposed clinical and radiographic degenerative spondylolisthesis(CARDS) classification system defines a distinct subset of patients with kyphotic angulation at the involved segment in lateral radiographs, as well as CARDS Type D spondylolisthesis. However, there is a paucity of research using CARDS classification to investigate patient-reported outcomes following TLIF. Thus, this study was performed to investigate the dynamic instability as well as postoperative patient reported outcomes in CARDS Type D slip versus none-Type D slip.

Materials/Methods: This study reviewed the charts and radiographs of a consecutive series of patients who received TLIF for L4/5 degenerative lumbar spondylolisthesis between July 2009 and July 2015. They were assigned into two groups based on preoperative lateral radiographs: Type D and non-Type D group. The translational motion was determined by comparing upright lateral radiograph (U) with a supine sagittal MR image (S) (combined, US) or flexion/extension radiographs (FE). Then, demographics as well as preoperative and postoperative patient reported outcomes (ODI and VAS back and leg pain scores) were collected.

Results: There were 34 and 163 patients in Type D and non-Type D group, respectively. In comparison with non-Type D group, the Type D group were characterized by prominently kyphotic angulation $\left(-2.7^{\circ}\right.$ vs. $\left.7.6^{\circ}, \mathrm{P}<0.05\right)$, collapse of ADH $(0.20$ vs. $0.28, \mathrm{P}<0.05)$ and higher degree of olisthesis $(22.4 \%$ vs. $19.3 \%, \mathrm{P}<0.05)$ on upright radiographs. Also, the Type D group demonstrated significantly higher translational motion $(11.3 \%$ vs. $7.1 \%, \mathrm{P}<0.001)$ on US analysis, but significantly lower translational motion (5.3\% vs. 7.6\%, $\mathrm{P}<0.001)$ on FE analysis. The preoperative ODI and VAS back pain scores were significant higher in CARDS Type D group than non-CARDS Type D group(P<0.05), and the presence of CARDS Type D slip and dynamic instability were associated with achieving minimal clinical important difference (MCID) in low back pain following lumbar fusion (all $\mathrm{P}$ value $<0.05$ ).

Discussion/Conclusion: CARDS Type D spondylolisthesis constitutes a distinct subset of patients associating with dynamic instability and worse preoperative patient reported outcomes. After TLIF, higher improvement in patient reported outcomes can be achieved in CARDS Type D versus none-type D spondylolisthesis.

022. Does the concomitant presence of scoliosis or spondylolisthesis affect outcomes following stability-preserving lumbar decompression for spinal stenosis?

Philip Louie, $M D^{l}$, Bryce Basques, $M D^{1}$, Michael Nolte, $M D^{2}$, Arya Varthi, MD ${ }^{3}$, Justin Paul, MD, PhD ${ }^{4}$, Steven Heidt, BS ${ }^{2}$, Comron Saifi, MD , Edward Goldberg, $M D^{6}$, Howard An, $M D^{1}$

${ }^{1}$ Rush University Medical Center, Chicago, IL,${ }^{2}$ Rush University Medical Center, Chicago, Illinois, ${ }^{3}$ Yale University , New Haven, CT, ${ }^{4}$, New York, NY, ${ }^{5}$ Midwest Orthopedics at Rush, Chicago, IL, ${ }^{6}$, Chicago, IL

Background/Introduction: Lumbar stenosis can be accompanied by degenerative scoliosis and spondylolisthesis, which complicates neural compression and makes deciding on a surgical treatment more complex. The purpose of this study was to compare the patient outcomes between lumbar decompression alone for patients with lumbar stenosis alone and those with stenosis and a concomitant scoliosis and/or spondylolisthesis.

Materials/Methods: We performed a retrospective analysis of patients who underwent a primary lumbar laminectomy. Patients were divided into three groups: those diagnosed with lumbar stenosis alone (without concomitant scoliosis or spondylolisthesis), those with a concomitant scoliosis ( $>10$ degrees of coronal angulation), and those with stenosis and a concomitant spondylolisthesis at the level of decompression). Lumbar plain radiographs were evaluated to assess for concomitant scoliosis and spondylolisthesis diagnoses. Patient reported outcomes were obtained in the form of Oswestry Disability Index (ODI) scores, Visual Analog Scales (VAS) scores for the back and leg, 12-Item Short Form Mental and Physical Survey (SF-12) scores, and the Veterans Rand 12-Item Health Mental and Physical Survey (VR-12) scores.

Results: Overall, 212 consecutive patients who fulfilled our inclusion criteria were assessed. All patients with a spondylolisthesis were found to be Meyerding Grade 1 and stable on flexion/extension lumbar plain radiographs. The patients in the scoliosis group were found to have significantly more patients with an American Society of Anesthesiologists (ASA) grade of 3 or greater (48.8\% vs 32.2\%; p=0.001) compared to the stenosis-only patients. Patients with scoliosis had a higher incidence of smokers $(\mathrm{p}=0.002)$ and had a shorter duration of symptoms then patients with stenosisalone $(29.3 \pm 5.9$ months vs $41.1 \pm 53.8$ months; $\mathrm{p}=0.042)$. There were no significant difference in any of the patient reported outcomes at preoperative and post-operative time points. Re-operation rates were not significantly different between the 3 groups.

Discussion/Conclusion: At approximately two years after lumbar decompression alone, the presence of concomitant scoliosis or stable spondylolisthesis in lumbar spinal stenosis patients do not appear impact clinical outcomes. Overall re-operation rates are low and similar between the three groups. Patients that present with scoliosis or a stable spondylolisthesis can expect similar improvement of symptoms, following stabilitypreserving lumbar decompression, as those that present with stenosis-alone.

023. Obesity is associated with inferior patient reported outcomes following surgery for degenerative lumbar spondylolisthesis: an analysis of the Quality Outcomes Database

Andrew Chan, $M D^{1}$, Erica Bisson, $M D, M P H^{2}$, Mohamad Bydon, M.D. ${ }^{3}$, Steven Glassman, $M D^{4}$, Kevin Foley, $M D^{5}$, Eric Potts, $M D^{6}$, Christopher Shaffrey, $M D^{7}$, Mark Shaffrey, $M D^{8}$, Domagoj Coric, $M D^{9}$, Praveen Mummaneni, $M D^{10}$

${ }^{I}$ Department of Neurological Surgery, University of California, San Francisco, San Francisco, CA, ${ }^{2}$ University of Utah, Salt Lake City, UT, ${ }^{3}$, Rochester, MN ${ }^{4}$, Louisville , Kentucky, ${ }^{5}$ Semmes-Murphey Neurologic Institute, University of Tennessee, DiscGenics, Salt Lake City, UT, ${ }^{6}$ Goodman Campbell Brain and Spine, Indianopolis, IN, ${ }^{7}$ University of Virginia Medical Center, Charlottesville, VA, ${ }^{8}$ Department of 
Neurological Surgery, University of Virginia, Charlottesville, VA, ${ }^{9}$ Carolina Neurosurgery \& Spine Associates, Charlotte, NC, ${ }^{10}$, San Francisco, California

Background/Introduction: In light of the recent differing findings following two randomized clinical trials on surgery for degenerative lumbar spondylolisthesis (DLS), there is a need for the identification of patients who may benefit least versus most from surgery.This study investigates the impact of obesity on patient reported outcomes (PROs) following surgery for DLS.

Materials/Methods: This was an analysis of 477 patients who underwent surgery for grade 1 DLS in a prospectively-collected national spine registry. For univariate comparisons, patients were stratified by BMI $\geq 30 \mathrm{~kg} / \mathrm{m} 2$ (obese) and $<30 \mathrm{~kg} / \mathrm{m} 2$ (non-obese). Baseline and one year follow-up parameters were collected. PROs included the North American Spine Society (NASS) satisfaction questionnaire, numeric rating scale (NRS) back pain, NRS leg pain, Oswestry Disability Index (ODI), and EuroQoL-5D (EQ-5D).

Results: We identified 224 obese $(47.0 \%)$ and 253 non-obese patients $(53.0 \%)$. Obese patients were younger $(60.0$ vs 63.3 years, p<0.01), more often had diabetes mellitus $(25.4 \%$ vs $10.7 \%$, p < 0.01$)$, and had higher ASA grades $(56.3 \%$ vs $32.8 \%$ with ASA grades 3 or 4 , p<0.01). Obese patients more often presented with sensory predominant symptoms $(46.4 \%$ vs $38.9 \%, \mathrm{p}=0.02)$ and were less independently ambulatory $(82.5 \%$ vs. $93.7 \%$, $\mathrm{p}<0.01)$. Obese patients more often underwent fusion surgery $(87.9 \%$ vs $78.3 \%, \mathrm{p}<0.01)$, had higher estimated blood loss $(302.9 \pm 327.5$ vs $213.3 \pm 227.0 \mathrm{ml}, \mathrm{p}<0.01)$, longer operative times (212.7 \pm 95.2 vs $177.2 \pm 80.4 \mathrm{~min}, \mathrm{p}<0.01)$, and longer hospitalizations (3.3 \pm 1.6 vs $2.9 \pm 2.0$ days, $\mathrm{p}<0.01)$. At baseline, obese patients had worse NRS back pain, ODI, and EQ-5D scores $(\mathrm{p}<0.05)$. Both cohorts improved significantly from baseline for back and leg pain, ODI, and EQ-5D at 12 months $(\mathrm{p}<0.01)$. At 12 months, fewer obese patients responded that surgery met their expectations $(64.4 \%$ vs. $70.1 \%, \mathrm{p}<0.01)$. In adjusted multivariate analyses, increasing BMI was associated with worse NRS leg pain, ODI, and EQ-5D at 12 months $(\mathrm{p}<0.05)$.

Discussion/Conclusion: Obesity is independently associated with inferior pain, disability, and quality of life 12 months post-operatively. Surgeons may want to discuss these findings with obese patients to help set appropriate expectations.

\section{Lumbar discectomy is associated with higher rates of lumbar fusion}

Hector Castillo, $B S^{1}$, Michael Lee, M.D. ${ }^{2}$, Lewis Shi, $M D^{3}$

${ }^{l}$ University of Chicago Pritzker School of Medicine, Chicago, IL, ${ }^{2}$ University of Chicago Medical Center, Chicago, IL, ${ }^{3}$, Chicago, IL

Background/Introduction: Lumbar disc herniation affects over three million people in the United States every year, and the rate of operation continually increases. Surgical discectomy is the most common method for management and treatment of lumbar disc herniation. One long term sequela for microdiscectomy is the risk of undergoing additional surgeries. There are very limited population-level studies that examine the rate of lumbar fusion following lumbar discectomy.

Materials/Methods: 10 Year Fusion After Discectomy Rates: The patients who had undergone discectomies were filtered in the Marketscan database via CPT codes specific for lumbar discectomy $(n=223,291)$. The group was then followed every year up to 10 years after the initial indexed lumbar discectomy dates for reoperation involving a lumbar spinal fusion CPT code. Fusion Rate Comparison: Study population only included patients who had a previous lumbar ICD-9 diagnosis in the Marketscan $(n=489,975)$. Patients were separated into two groups based on whether or not they had undergone lumbar discectomy. Pearson Chi-Squared Test was utilized to assess significance when comparing the proportion of patients who receive lumbar fusion in each arm.

Results: For the first part of this study, the rate of fusion ranged between $1.69 \%$ (1 year time frame following discectomy) to $8.50 \%$ ( 10 year time frame following discectomy). When comparing the two cohorts in the second part of this study, the fusion rates were $12.50 \%$ for the discectomy group and $4.19 \%$ for the non-discectomy group $(\mathrm{p}<.0001, \alpha=.05)$. Patients who had a lumbar discectomy procedure were $2.97(95 \% \mathrm{CI}[2.86,3.10])$ times more likely to undergo a lumbar fusion than those who had not undergone a lumbar discectomy in the past.

Discussion/Conclusion: This is the largest population study to explore the rate of lumbar fusion following lumbar discectomy and the first to conclude that lumbar discectomy is statistically associated with an increased likelihood of undergoing a lumbar fusion in the future. Patients who had previously undergone a lumbar discectomy were roughly three times more likely to undergo a lumbar fusion procedure than a patient who had not undergone a previous lumbar discectomy. This finding can be an important supplement for the physician-patient discussion regarding expectations and potential for re-operation.

\section{Early healthcare utilization after primary lumbar decompression/discectomy predicts revision surgery at two years}

Nikhil Jain, $M D^{l}$, Jack Xie, $B S^{2}$, Frank Phillips, $M D^{3}$, Safdar Khan, $M D^{4}$, Elizabeth $Y u, M D^{l}$

${ }^{1}$ Ohio State University, Columbus, Ohio,${ }^{2}$, Columbus, Ohio, ${ }^{3}$, Chicago, $I L,{ }^{4}$, Columbus, $\mathrm{OH}$

Background/Introduction: Various patient and clinical risk factors for reoperation in patients undergoing decompression and discectomy have been reported from numerous studies. However, whether early ( $<3$ months) healthcare utilization after lumbar decompression/discectomy predicts risk of reoperation has not been investigated.

Materials/Methods: Insurance claims data (2007 - Q3 2015) was used to study patients who underwent primary lumbar discectomy for herniated disc, or decompression for spinal stenosis. Patients who had early revision surgery ( $<3$ months), or who did not have continuous insurance enrollment for six-months pre-operatively and twenty-four months post-operatively were excluded. The first occurrence of delayed reoperation (between 6 and 24 months) was queried from the database and served as the primary endpoint. We defined high healthcare users as patients who had advanced imaging (MRI, CT scan) or electrodiagnostic studies, epidural injections, two or more emergency department (ED) visits or office visits 
longer than 30 minutes in the first three-months after index surgery. Additionally, we studied failure to initiate physical therapy, and failure of wean off narcotics as possible risk factors. Log rank survival curves were generated to depict the survival (freedom from reoperation) at two-years. To analyze significance of risk factors for delayed reoperation, a Cox proportional hazards model was used to study above mentioned variables after adjusting for other confounders. Adjustment for age, gender, ethnicity, comorbidity burden, type of procedure, and number of levels was done.

Results: We included a total of 10,384 patients with a mean age of $65.6 \pm 10.7$ years. The cumulative delayed reoperation rate at two-years in our cohort was $5.6 \%(581 / 10,384)$. Demographic and clinical profile of subgroups (delayed reoperation vs. no reoperation) has been described. Survival curves with cox hazards have been given in Fig 1.

Discussion/Conclusion: The aim of our study was to see if early healthcare utilization patterns after primary lumbar decompression/discectomy are predictive of delayed reoperation at two-years. High healthcare utilization (imaging, injections, ED visits, prolonged office visits, electrodiagnostic studies), and inability to wean off narcotics within the first 3-months predicted delayed reoperation at two-years. Additionally, initiation of guided physical therapy within first 3-months was not associated with lower risk of reoperation.

026. Patients undergoing revision microdiscectomy for recurrent lumbar disc herniation experience worse clinical outcomes and more re-operations compared to patients undergoing a primary microdiscectomy

Bryce Basques, $M D^{1}$, Philip Louie, $M D^{1}$, Michael Nolte, $M D^{2}$, Steven Heidt, BS ${ }^{2}$, Deven Carroll, MS ${ }^{3}$, Arya Varthi, MD ${ }^{4}$, Justin Paul, MD, PhD , Edward Goldberg, $M D^{6}$, Howard An, $M D^{l}$

${ }^{1}$ Rush University Medical Center, Chicago, IL, ${ }^{2}$ Rush University Medical Center, Chicago, Illinois, ${ }^{3}$, Chicago, Illinois, ${ }^{4}$ Yale University , New Haven, CT, ${ }^{5}$, New York, NY, ${ }^{6}$, Chicago, IL

Background/Introduction: In the setting of a revision discectomy surgery for a recurrent disc herniation, outcomes can be difficult to predict. Some studies with smaller patient populations and diverse outcome protocols have not found significant clinical outcome differences between these two patient populations. The goal of this study is the compare the demographics and outcomes of patients undergoing primary lumbar microdiscectomy surgery for a disc herniation and those undergoing a revision microdiscectomy surgery for a recurrent disc herniation.

Materials/Methods: We performed a retrospective analysis of patients who underwent a primary or revision lumbar microdiscectomy. Patients were divided into two groups: those undergoing a primary surgery for a lumbar disc herniation (primary) and those undergoing a revision procedure for a recurrent disc herniation (recurrent). Patient reported outcomes were obtained in the form of Oswestry Disability Index (ODI) scores, Visual Analog Scales (VAS) scores for back and leg, 12-Item Short Form Mental and Physical Survey (SF-12) scores, and the Veterans Rand 12-Item Health Mental and Physical Survey (VR-12) scores. Patients were also surveyed about their expectations and satisfactions following surgery.

Results: Overall, 296 consecutive patients who fulfilled our inclusion criteria were assessed. The patients in the recurrent group were significantly younger than those in the primary group ( $44.6 \pm 12.7$ vs $53.2 \pm 16.2$ years; $p<0.001)$. Average follow-up in the primary group was 24.1 months (range 6-78 months), while average follow-up was 30.1 months (range 3-96 months) in the recurrent group. On average, patients underwent a revision microdiscectomy 46.1 months after their primary microdiscectomy. Patients presenting with a recurrent disc herniations experienced symptoms for significantly less time before surgery compared the those undergoing their first surgery $(5.2 \pm 1.0$ vs $28.3 \pm 2.8$ months; $<<0.001)$. Patients who underwent a revision microdiscectomy for a recurrent lumbar disc herniation presented with significantly worse post-operative VASback $(7.1 \pm 3.1$ vs $3.2 \pm 2.8 ; p=0.003)$, VAS-leg $(7.3 \pm 3.2$ vs $2.7 \pm 3.0 ; p=0.001)$, and ODI $(49.3 \pm 19.8$ vs $24.6 \pm 19.6$; $p=0.003)$ scores. The patients in the recurrent group underwent significantly more additional surgeries (after the current revision procedure) [25.5\% vs $7.0 \% ; \mathrm{p}=0.009]$.

Discussion/Conclusion: Revision lumbar microdiscectomy patients for recurrent disc herniation experience worse clinical outcomes and higher risks of re-operation compared to patients undergoing a primary microdiscectomy.

027. Opioids delay healing of spinal fusion: a rabbit posterolateral lumbar fusion model

Nikhil Jain, $M D^{1}$, Khaled Himed, $B S^{2}$, Jeffrey Toth, $P h D^{3}$, Karen Briley, PhD ${ }^{2}$, Frank Phillips, $M D^{4}$, Safdar Khan, $M D^{5}$

${ }^{1}$ Ohio State University, Columbus, Ohio, ${ }^{2}$ The Ohio State University, Columbus, Ohio, ${ }^{3}$ Medical College of Wisconsin, Milwaukee, Wisconsin, ${ }^{4}$, Chicago, IL, ${ }^{5}$, Columbus, $\mathrm{OH}$

Background/Introduction: Opioid use is prevalent for management of pre- and post-operative pain in patients undergoing spinal fusion. In-vitro and pre-clinical studies suggest a negative effect of opioids on bone healing and turnover. However, the effect of opioids on healing of spinal fusion has not been investigated before. The objective of our study was to study the effect of systemic opioids on the healing of spinal fusion using a rabbit posterolateral spinal fusion model.

Materials/Methods: 24 adult, New Zealand white rabbits were studied in two groups. The opioid group (n=12) received four-weeks preoperative and six-weeks post-operative transdermal fentanyl. The control group $(n=12)$ received only peri-operative pain control as necessary. All animals received a bilateral L5-L6 posterolateral spinal fusion using iliac crest autograft. Animals were euthanized at the six-week post-operative time point, and assessment of fusion was done by manual palpation, plain radiographs, micro-computed tomography (microCT) using previously reported scoring systems, and histological analysis.

Results: 12 animals in control group and 11 animals in the opioid group were available for analysis at the end of six weeks. The mean serum fentanyl level in the opioid group at pre-operative assessment (before skin incision) was $2.73 \pm 0.24 \mathrm{ng} / \mathrm{ml}$, and $1.58 \pm 0.71 \mathrm{ng} / \mathrm{ml}$ four-weeks postoperatively. The fusion scores on manual palpation, radiographs, and microCT were not statistically different. Three-dimensional microCT morphometry found that the fusion mass in the opioid group had a lower trabecular number $(\mathrm{p}=0.02)$, higher trabecular separation ( $\mathrm{p}=0.02)$, and lower bone volume $(\mathrm{p}=0.09)$ as compared to control. On histological analysis in the control group, there was remodeling of woven bone to lamellar 
organization with incorporation of osteocytes, and formation of mature marrow. In the opioid group, there were no sections showing lamellar organization and development of mature marrow elements (Fig 1). Less dense trabeculae on microCT correlated with histological findings of relatively immature fusion mass in the opioid group.

Discussion/Conclusion: Systemic opioids in the pre- and post-operative period negatively affects the process of spinal fusion healing. Our findings indicate a less mature and inferior quality fusion mass because of opioids, and lay foundation for further research.

\section{Opioid utilization following lumbar arthrodesis: trends and factors associated with long-term use}

Piyush Kalakoti, $M D^{1}$, Nicholas Bedard, $M D^{2}$, Nathan Hendrickson, $M D, M S^{3}$, Comron Saifi, $M D^{4}$; Andrew Pugely, $M D^{5}$

${ }^{1}$ University of Iowa, Iowa City, IA, ${ }^{2}$ University of Iowa, Iowa City, Iowa, ${ }^{3}$ Department of Orthopedics and Rehabilitation, University of Iowa, Iowa City, IA, ${ }^{4}$ Midwest Orthopedics at Rush, Chicago, IL, ${ }^{5}$, New York, $N$

Background/Introduction: Limited or no data exist evaluating long-term utilization rates and risk-factors associated with prolonged opioid use following lumbar spine surgery. The authors investigate trends in post-operative narcotic use amongst preoperative opioid users (OU) versus nonopioid users (NOU), and identify factors associated with postoperative narcotic use at 1-year following lumbar arthrodesis.

Materials/Methods: In an observational cohort study, we identified patients registered in the Humana Inc. claims dataset (2007 through 2016Q3)that underwent anterior (ALIF) or posterior/transforaminal lumbar interbody fusions (P/TLIFs), postero-lateral fusion (PLF) or a combination of ALIF and PLF. Based on preoperative opioid-use, patients were identified as an OU (history of narcotic prescription filled within 3months before surgery) or a NOU (no preoperative prescription). Rates of opioid use were evaluated pre-operatively for OU, and trended for 1-year post-operatively for both OU and NOU. Multivariable regression techniques investigated factors associated with the use of narcotics at 1-year following lumbar surgery.

Results: Overall, 26,553 patients (OU: 58.3\%) underwent lumbar surgery (ALIF:8.5\%; P/TLIF:43.8\%; PLF:41.5\%; ALIF+PLF:6.2\%). At onemonth following surgery, $60.2 \%$ NOU and $82.9 \%$ OUs had a filled opioid prescription. At 3-months, rates of prescription opioids declined significantly to $13.9 \%$ in NOUs vs $53.8 \%$ in OUs, while plateauing at 6-12-month postoperative period (NOU:8.4-9.6\%; OU:42.1-45.3\%). At 1-year, significantly higher narcotic prescription filling rates was observed in OUs compared to NOUs (42.4\% vs 8.6\%; $<0.001)$.[Figure 1] Preoperative opioid use was a significant driver of 1-year narcotic use following ALIF (OR:7.1;p<0.001) along with younger age[<=50 years] (OR:1.3;p=0.05), male gender $(\mathrm{OR}: 1.2 ; \mathrm{p}=0.05)$, history of drug dependence $(\mathrm{OR}: 2.1 ; \mathrm{p}=0.038)$, lower back pain $(\mathrm{OR}: 2.0 ; \mathrm{p}=0.022)$, and depression/anxiety disorder (OR:1.3; $<0.001)$.

Discussion/Conclusion: Approximately one-third patients used opioids prior to lumbar spine surgery. Postoperative opioid-use fell dramatically during the first 3-months in NOU, but nearly half of the pre-op opioid users will remain on narcotics at 1-year postop. Our findings serve as a baseline in identifying patients at risk for chronic use and encourage discontinuation of opioids prior to lumbar spinal surgery owing to the risks associated with it.

\section{The impact of opioid prescription in patients undergoing decompression laminectomy of the lumbar spine}

Stephanie Kha, BS ${ }^{l}$, Sara Davin, PsyD, MPH ${ }^{2}$, Judith Scheman, PhD ${ }^{3}$, Edward Benzel, M.D. ${ }^{4}$

${ }^{1}$, Cleveland Heights, $\mathrm{OH},{ }^{2}$ Cleveland Clinic, Center for Neuro-Restoration, Cleveland, $\mathrm{OH},{ }^{3}$ Cleveland Clinic, Digestive Disease and Surgery Institute, Cleveland, $\mathrm{OH},{ }^{4}$ Cleveland Clinic Foundation, Neurological Institute, Cleveland, $\mathrm{OH}$

Background/Introduction: Post-operative pain management for patients with a history of opioid usage remains a challenge for spine surgeons. Opioid medications are controversial in this setting due to physiological side effects and the potential for abuse, diversion, tolerance, and addiction. This study aims to characterize the pattern of opioid utilization in patients undergoing lumbar spine surgery and compare the post-operative course between patients with and without pre-operative opioid prescriptions.

Materials/Methods: Electronic medical records were reviewed for patients (age>18years) with a diagnosis of lumbar spinal stenosis undergoing single- or multi-level decompression laminectomy in 2011 at our institution. Data regarding patient demographics, surgical levels operated, preoperative and post-operative medications, and hospital course and outcome were collected. Primary outcome measures were length of in-hospital stay and date of discontinuation for post-operative opioid prescription. Student's t-tests and Chi-squared tests were performed for statistical analyses.

Results: Seventy-one patients were included in this review. Forty patients $(56 \%)$ had a pre-operative opioid prescription documented six months before date of surgery. Thirty-one patients (44\%) did not have record of a pre-operative opioid prescription. Average number of operative levels for decompression laminectomy was 2 levels for both groups. The pre-operative opioid group compared to the no pre-operative opioid group had a greater proportion of females ( $48 \%$ vs. 39\%), younger mean age (63 years vs. 66 years; $p=0.048$ ), higher frequency of concomitant prescription with pre-operative benzodiazepines ( $23 \%$ vs. $16 \%$; $\mathrm{p}=0.561)$, longer average length of stay in the hospital ( 3.70 days vs. 3.06 days; $\mathrm{p}=0.073)$, and longer duration on opioids prescribed after surgery (185 days vs. 87 days; $\mathrm{p}=0.013$ ). A summary of the results are listed in Table 1 .

Discussion/Conclusion: Patients on opioid medications prior to spine surgery are more likely to have a longer hospital stay and continue on opioids for a longer time after surgery, compared to patients who do not have a pre-operative opioid prescription. Spine surgeons, in partnership with pain management specialists, should seek to identify these patients before surgery and evaluate strategies to optimize the post-operative course.

030. The relationship of post-operative opioids and HCAHPS Pain Management Scores in lumbar spine surgery patients

Robert Winkelman, $B S^{1}$, Jay Levin, $B S^{2}$, Joseph Tanenbaum, $B S^{3}$, Thomas Mroz, M.D. ${ }^{4}$, Michael Steinmetz, MD 


\author{
${ }^{l}$ Carnegie Mellon University, Cleveland Heights, Ohio, ${ }^{2}$, Cleveland, OH, ${ }^{3}$ Case Western Reserve University School of Medicine, Cleveland, \\ Ohio, ${ }^{4}$ Cleveland Clinic Foundation, Neurological Institute, Cleveland, OH, ${ }^{5}$ Cleveland Clinic, Department of Neurosurgery , Cleveland, OH
}

Background/Introduction: The recent use of patient experience as a quality metric in healthcare has generated considerable concern amongst medical providers. One major concern is the Hospital Consumer Assessment of Healthcare Providers and Systems (HCAHPS) survey may be incentivizing providers to prescribe excessive amounts of opioids to patient in an effort to bolster patient experience scores. Despite the significance of a potential relationship between HCAHPS scores and opioids, there are presently no reports in the medical literature that have studied the association of these two important subjects. The goal of our study was to investigate the relationship of post-operative opioid prescriptions and Pain Management scores on the HCAHPS survey in lumbar spine surgery patients.

Materials/Methods: The present study is a retrospective cohort analysis of 210 patients who underwent lumbar spine surgery at the Cleveland Clinic from 2013-2015 and completed an HCAHPS survey. The primary outcome of interest was the pain management score on the HCAHPS survey. A multivariate regression model was developed to investigate the association between patients' pain management scores and the average daily dose of opioids prescribed within 6 weeks following surgery. The average daily dose of opioids prescribed in the 6 weeks prior to surgery as well as several other demographic variables (e.g. age, sex, health status) were also included as covariates in the model.

Results: The results of the regression analysis indicate that an increase of 15 morphine equivalents for the average daily dose of post-operative opioids is associated with a $25.8 \%$ (95\% CI: 12.8 to $45.2 \%$ ) increase in the predicted number of pain management points lost when all other covariates in model are held constant. In contrast, a similar increase in the average daily dose of pre-operative opioids was associated with nonsignificant changes in the predicted number of pain management points lost(-0.1\%; $95 \%$ CI:-32.9 to $18.1 \%)$.

Discussion/Conclusion: The results from this study suggest that the average daily dose of opioids prescribed post-operatively is negatively correlated with pain management scores on the HCAHPS survey in lumbar spine surgery patients. These findings challenge the conventional belief of many healthcare providers who associate larger doses of opioids with higher patient pain management scores.

\title{
031. Predictive analytics for determining extended operative time in corrective ASD surgery
}

Peter Passias, $M D^{1}$, Gregory Poorman, $B A^{2}$, Dennis Vasquez-Montes, $M S^{1}$, Samantha Horn, $B A^{1}$, Frank Segreto, BS ${ }^{1}$, Cole Bortz, BA ${ }^{1}$, Virginie Lafage, $P h D^{3}$, Christopher Ames, $M D^{4}$, Michael Gerling, $M D^{5}$; Eric Klineberg, $M D^{7}$

${ }^{1}$, New York, NY ${ }^{2}$, bronxville, ny, ${ }^{3}$ Hospital for Special Surgery, New York, NY, ${ }^{4}$ University of California San Fransisco, San Fransisco, CA, ${ }^{5}$, TriBeCa, NY, ${ }^{6}$ Johns Hopkins Hospital, Baltimore, Maryland, ${ }^{7}$ University of California Davis, Sacramento, California

Background/Introduction: More sophisticated techniques for surgical correction of adult spinal deformity (ASD) have increased op-times, which are correlated with higher complication incidence. This study employs a statistical learning algorithm to determine key variables affecting optime.

Materials/Methods: Retrospective review of prospective multi-center database, cases between 2008-2016 as part of the International Spine Study Group. Conditional Inference Decision Trees assessed factors affecting skin-to-skin op-time and the cut-off points at which factors have a global effect. A Conditional Variable-Importance table was constructed based on a non-replacement sampling set of 2000 Conditional Inference trees. Following identification of the top 15 influencing factors, means comparison for each variable at significant cut-offs indicated variable effect size.

Results: 544 surgical patients undergoing ASD-corrective surgery(58.0yrs, mean 11.3 levels fused, mean op-time:377.6 min) were included. The strongest predictor for op-time was institution/surgeon. Grouped by decision tree hierarchy, center/surgeons a and b were on average 2 hours faster than center/surgeons c-f, which were 43 minutes faster than centers $g$-j(all $\mathrm{p}<0.001)$. Other key predictors were, in order, approach(combined vs. posterior increases time by 139 minutes, $\mathrm{p}<0.001)$, number of levels fused $(<4$ vs. $5-9$ increased time by 68 minutes, $p<0.050 ; 5-9$ vs. $>10$ increased time by 47 minutes, $p<0.001$ ), age (age $>50$ increases time by 57 minutes, $p<0.001$ ), and patient frailty(score $>1.54$ increases time by 65 minutes,p<0.001). Surgical techniques, including three-column osteotomies(35 minutes), interbody device(45 minutes), and decompression(48 minutes), also increased op-time. Minor and major complications were correlated with $>66$ minutes of increased op-time. Increased op-time also correlated with increased LOS, EBL, and inferior 2-year ODI scores.

Discussion/Conclusion: Procedure location and specific surgeon are key factors determining op-time accounting for operative time increases of more than two hours. Surgical approach and number of levels fused were next most significant in elongating surgeries, respectively. Extended optime correlated with longer length of hospital stay, higher EBL, and inferior 2-year improvement in ODI.

032. Treatment of the fractional curve only in adult scoliosis: comparison to lower thoracic and upper thoracic fusions

Dominic Amara, $B A^{1}$, Sigurd Berven, $M D^{2}$, Christopher Ames, $M D^{3}$, Bobby Tay, $M D^{2}$, Vedat Deviren, $M D^{2}$, Shane Burch, $M D^{2}$, Praveen Mummaneni, $M D^{4}$; Dean Chou, $M D^{5}$

${ }^{1}$ University of California, San Francisco, SAN FRANCISCO, California, ${ }^{2}$, San Francisco, CA, ${ }^{3}$ University of California San Fransisco, San Fransisco, CA, ${ }^{4}$, San Francisco, California, ${ }^{5}$ University of California, San Francisco, San Francisco, California

Background/Introduction: Significant variability in strategies for surgical management of adult spinal deformity. Radiculopathy from the fractional curve, typically L4-S1, is frequently a reason for scoliosis patients to pursue surgical intervention. The purpose of this paper is to evaluate treatment outcomes of limited fusion of the fractional curve (FC) only compared to treatment of the entire deformity with long fusion. 
Materials/Methods: 99 consecutive adult scoliosis patients from 2012-2016 were retrospectively studied at our institution. Patients with fractional curves from L4 to $\mathrm{S} 1>10^{\circ}$ underwent 3 categories of surgeries: 1) fractional curve only (FC, n=27), 2) lower thoracic to sacrum (LT, $\mathrm{n}=46$ ), or 3 ) upper thoracic to sacrum (UT, n=26). Primary outcomes were the rates of surgical revision surgery and complications. Secondary outcomes were estimated blood loss, length of hospital stay, and discharge destination.

Results: There were no significant preoperative differences in age, gender, smoking status, prior operation, fractional curve degree, pelvic tilt (PT), sagittal vertical axis (SVA), coronal balance, pelvic incidence/lumbar lordosis mismatch (PI-LL), or the proportion of balanced spines $\left(\mathrm{SVA}<5 \mathrm{~cm}, \mathrm{PI}-\mathrm{LL}<10^{\circ}\right.$ and $\left.\mathrm{PT}<20^{\circ}\right)$ between the three treatment groups. Mean follow-up was 30.3 (range 12-101) months. The FC group had a lower complication rate $(22 \%$ [FC] vs $57 \%$ [LT] vs $58 \%$ [UT], p=0.009), but a higher rate of extension surgery $(26 \%$ [FC] vs $13 \%$ [LT] vs $4 \%$ [UT], $\mathrm{p}=0.068$ ). The respective (FC, LT, UT) average estimated blood loss (593cc vs $1950 \mathrm{cc}$ vs $2634 \mathrm{cc}, \mathrm{p}<0.001)$, length of hospital stay (5.7 vs 8.3 vs 8.3 days, $\mathrm{p}=0.002)$ and rate of discharge to acute rehabilitation ( $30 \%$ vs $45 \%$ vs $85 \%$, $\mathrm{p}<0.001$ ) were all lower for FC and highest for UT.

Discussion/Conclusion: Treatment of the FC only is associated with a lower complication rate, shorter hospital stay and lower blood loss than complete scoliosis treatment. However, there is a higher associated rate of extension of the construct to the LT or UT levels, and patients should be counseled when considering their options.

033. Differences in primary and revision deformity surgeries: following 1,063 primary thoracolumbar adult spinal deformity fusions over time

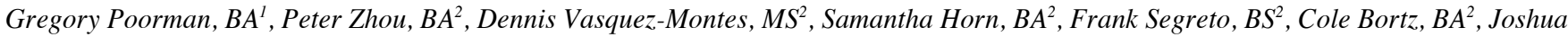
Auerbach, $M D^{3}$; Michael Gerling, $M D^{4}$, Justin Paul, $M D, P h D^{2}$, Peter Passias, $M D^{2}$

${ }^{1}$, bronxville, ny, ${ }^{2}$, New York, $N Y,{ }^{3}$, Bronx, $N Y,{ }^{4}$, TriBeCa, NY

Background/Introduction: Adult spinal deformity (ASD) is a common pathology that can lead to decreased quality of life, pain, physical limitations, and dissatisfaction with self-image. Durability of interventions for deformity treatment is of paramount concern to surgeons, as revision rates remain high. The aim of this study was to describe properties of ASD revisions relative to primary surgeries, and determine clinical variables that can predictor revision.

Materials/Methods: Retrospective review. Patients undergoing thoracolumbar fusion, five or more levels, for scoliosis (primary diagnosis ICD9 737.x) were identified on a state-wide database. Primary and Revision (returning for refusion procedure) surgeries were compared based on demographic, hospital stay, and clinical characteristics. Differences between primary and revision surgeries, and predictors of primary surgeries requiring revision, utilized binary logistic regression controlling for age, comorbidity burden, and levels fused. Significance was set to $\mathrm{p}<0.05$.

Results: 1063 patients (average 7.4 levels fused, mean age: 47.6 years, 69.0\% female) who underwent operative treatment for ASD were identified, of which 123 (average 7.1 levels fused, 11.6\%, mean age 61.43, 80.5\% female) had revisions. Primary surgeries were 0.3 levels longer $(\mathrm{p}=0.013$ ), used interbody $\sim 11 \%$ more frequently $(\mathrm{p}=0.020$ ), and used BMP $\sim 12 \%$ less frequently ( $\mathrm{p}=0.008$ ). Revisions occurred 176.4 days after the primary on average. The most frequent causes of revisions were: $43.09 \%$ implant failure, $24.39 \%$ acquired kyphosis, and $14.63 \%$ enduring scoliosis. After controlling for age, comorbidities, and levels fused older, more comorbid, female, and white-race patients were more likely to be revised. Upon multivariate regression, after controlling for age and levels fused, overall complications remained non-different (OR: 0.8, 95\% CI: 0.6-1.2). However, revision remained an independent predictor for infection (OR: 5.5, 95\% CI: 2.8-10.5).

Discussion/Conclusion: In a statewide database with individual patient follow up of up to 4 years, $10 \%$ of ASD patients undergoing scoliosis correction required revision. Revision surgeries had higher infection incidence. These results can be used to better inform patient selection and improve durability of ASD-corrective surgery

\section{Derivation and validation of a predictive app for 30-day readmission risk following lumbar spine surgery}

Piyush Kalakoti, $M D^{1}$, Alan Shamrock, $M D^{2}$, Christian Bowers, $M D^{3}$, Meic Schmidt, $M D^{3}$, Daniel Sciubba, $M D^{4}$; $\underline{\text { Andrew Pugely, } M D^{5}}$

${ }^{1}$ University of Iowa, Iowa City, IA, ${ }^{2}$ University of Iowa, Iowa City, Iowa, ${ }^{3}$ New York Medical College, Westchester Medical Center \& Health Network, Valhalla, NY, ${ }_{4}^{4}$ Johns Hopkins Hospital, Baltimore, Maryland, ${ }^{5}$, New York, NY

Background/Introduction: Recent seismic reforms in healthcare levies financial penalties on hospitals based on their performances that includes assessment of readmission rates. To optimize outcomes and enhance quality of care delivered to spine patients, it is critical to identify inherent risks associated with readmissions to implement appropriate preventive measures for modifiable risk-factors. The current study seeks to develop and validate a predictive model of 30-day readmission risk in patients undergoing lumbar surgery.

Materials/Methods: Utilizing the National Readmission Database (NRD), we identified adult patients undergoing lumbar surgery. Multivariable regression techniques, generalized estimating equations(GEE) to control clustering of outcomes by hospitals, were employed to create a predictive model identifying factors independently associated with the risk of 30-day readmission. Internal validation of the model was performed by evaluating the impact on c-statistics(area under curves) using 50-bootstrapped replacement samples.

Results: 0verall, 18416 patients (median age:60 years; $48 \%$ male) registered in the NRD underwent lumbar surgery(primary surgery: $91.6 \%$; revision: $8.4 \%)$. Of these, $1623(8.8 \%)$ were readmitted within 30-days. Patients undergoing revision lumbar surgery had higher readmission rates compared to primary lumbar surgery $(11.7 \%$ vs $8.6 \%$; $<0.001)$. Most common causes for readmissions were wound complications or abscess $(19.8 \%)$, implant related complication(3.3\%), exacerbation of acute postoperative pain/low back pain (4.0\%) and pulmonary embolism $(2.6 \%)$. Factors associated with decreased likelihood od readmission include private insurance(OR:0.7;p=0.009) and higher income(OR:0.8; $=0.004)$. Advancing age(OR:1.01;p=0.004), Medicaid(OR:1.4; $=0.003)$, weekend admissions(OR:1.2;p=0.018), revision lumbar 
surgery(OR:1.3; $=0.002)$, comorbidities [morbid obesity(OR:1.2; $\mathrm{p}=0.019)$, uncontrolled diabetes(OR:1.5; $\mathrm{p}=0.002)$, hypertension $(\mathrm{OR}: 1.2 ; \mathrm{p}=0.026)$, osteoporosis(OR:1.3; $\mathrm{p}=0.002)$, fluid and electrolyte disorder(OR:1.3; $=0.002)$ ] and post-surgical complications [neurological complication including dural tears and CSF leak (OR:1.6;p=0.004), wound complication(OR:1.6; $\mathrm{p}=0.002)$, venous thromboembolism(OR:1.7; $\mathrm{p}=<0.001)$, acute renal failure $(\mathrm{OR}: 1.3 ; \mathrm{p}=0.022)$ ] were associated with higher odds of readmission. [Figure 1]. A validated web-based version of model predicting the risk of readmission based on patient and hospital characteristics was developed, and can be accessed at the following link: https://www.neurosurgerycost.com/spine/spinesurgery

Discussion/Conclusion: The study quantifies risk estimates associated with the risk of 30-day readmission in patients undergoing lumbar surgery. The proposed validated web-based tool(app) can potentially be utilized by patients, providers, stakeholders and policy makers to assess individualized risks, shared decision making and guiding the process of patient counselling and informed consent.

\section{Complications of spine surgery in super obese patients}

Gennadiy Katsevman, $M D$, Scott Daffner, MD, Sanford Emery, MD, MBA, John France, MD, Cara Sedney, MD, MA

\section{West Virginia University, Morgantown, WV}

Background/Introduction: Super obese patients (defined as BMI $\geq 50 \mathrm{~kg} / \mathrm{m} 2$ ) likely experience different risks in spine surgery when compared to patients of lesser weight. Although increasing in prevalence, there is a lack of research specifically regarding spine surgery in super obese patients.

Materials/Methods: All patients undergoing spine surgery at a single academic medical center with a BMI $\geq 50$ from 2012-2016 were included for review. Age, gender, BMI, surgical indications, complications, mortality, and procedure types were recorded. Based upon pre-hoc power analysis, a 1:1 ratio of age-matched control patients of BMI $<50$ who underwent spine surgery were selected from the same time period for comparison. The groups were compared with respect to complication rate as the primary outcome and mortality rate as the secondary outcome. Elective and nonelective cases were also considered separately. Categorical variables were compared using chi-square analysis with significance set as $p<0.05$.

Results: We identified 63 patients in the super obese group who underwent 86 surgeries and 60 patients (64 surgeries) in the control group. Age and the number of elective and non-elective cases were not significantly different between groups. Mean BMI of the super obese group was 55 (range 50-77) compared to 29 in the control group (range 20-49). Super obese patients had higher complication rate [30\%(N=19) vs 10\%(N=6), $\mathrm{p}=0.0055]$ but similar 30 day mortality rate (5\% vs $5 \%$ ) compared to controls. For both groups, the complication rate of non-elective surgery was greater than elective surgery, although not significantly so (super obese $\mathrm{p}=0.1035$, control $\mathrm{p}=0.1944)$. Complication rates for elective surgery were $21 \%$ ( $\mathrm{N}=7$ ) for super obese patients and $4 \%(\mathrm{~N}=1)$ for controls $(\mathrm{p}=0.0507)$; complication rates for non-elective procedures were $40 \%(\mathrm{~N}=12)$ and $14 \%(\mathrm{~N}=5)$, respectively ( $\mathrm{p}=0.015)$. All mortalities in both groups involved non-elective cases.

Discussion/Conclusion: Super obese patients should be considered separately from other patient populations with respect to spine surgery. The complication rate is significantly higher in this population, particularly for non-elective cases. Super obese patients should be counseled regarding these risks, and steps should be taken to mitigate risks whenever possible in this population.

\section{Preoperative high, as well as low, platelet counts correlate with perioperative adverse outcomes after posterior lumbar surgery}

\section{Rohil Malpani, B.S. ${ }^{1}$, Michael Clark, B.S. ${ }^{2}$, Patawut Bovonratwet, $B S^{3}$, Jonathan Grauer, $M D^{2}$}

\section{${ }^{1}$ Yale School of Medicine, New Haven, Connecticut, ${ }^{2}$, New Haven, CT, ${ }^{3}$ Yale School of Medicine, New Haven, CT}

Background/Introduction: It is generally assumed that preoperative low platelet counts correlate with adverse outcomes after lumbar surgeries. This has not been quantified, and the effect of varying platelet counts has not previously been assessed in a large patient population, especially on the high end of the platelet spectrum. The aim of this study is to use postoperative adverse event data to risk stratify posterior lumbar surgery $+/$ - fusion in patients with varying preoperative platelet counts to address these questions.

Materials/Methods: Patients who underwent non-emergent, posterior lumbar surgery +/- fusion between 2011-2016 were identified from the American College of Surgeons National Surgical Quality Improvement Program (ACS-NSQIP) database. A histogram of platelet counts was made and overlaid with adverse event data as a function of platelet count. Adverse events studied included: any, major, minor adverse events, hospital length of stay, and hospital readmissions. Patients were classified into platelet categories based on determining upper and lower bounds on when the adverse outcomes crossed a relative risk of 1.5. Then, univariate and multivariate analyses were performed to quantify the risks.

Results: Based on inclusion/exclusion criteria, 137,714 patients were identified after excluding $10.61 \%$ of patients who not have platelet data. Patients were classified into normal, abnormally low $(\leq 140,000 / \mathrm{mL})$ and abnormally high $(\geq 447,000 / \mathrm{mL})$ platelet categories using the relative risk threshold of 1.5 for any adverse event (Figure). Based on the platelet categories defined above, multivariate analysis revealed the abnormally low platelet cohorts to be associated with a higher likelihood of adverse events (any [OR=1.37, $\mathrm{p}=<0.001]$, major $[\mathrm{OR}=1.40, \mathrm{p}=<0.001]$ and minor $[\mathrm{OR}=1.28, \mathrm{p}=0.002]$ adverse events), and a higher risk of readmission [OR=1.30, $\mathrm{p}=<0.001]$. On the other hand, the abnormally high platelet group was associated with an even higher risk (any $[\mathrm{OR}=1.61, \mathrm{p}=<0.001]$, major [OR=1.70, $\mathrm{p}=<0.001$ ] and minor [OR=1.33, $\mathrm{p}=0.048]$ adverse events). Additionally, both groups were associated with a longer hospital length of stay (two-sample t-test $\mathrm{p}<0.001$ ).

Discussion/Conclusion: The cutoff values for platelets leading to 1.5 greater relative risk of adverse outcomes after posterior lumbar surgeries mirrored those found in the literature. Further, the current study quantified the increased risks of perioperative adverse outcomes for patients with abnormally low and abnormally high platelet values.

037. The utility of intraoperative electromyography and computer-assisted navigation for lumbar pedicle screw placement 


\section{${ }^{1}$ UCLA Medical Center, Los Angeles, CA,${ }^{2}$, Redwood City, California, ${ }^{3}$ Stanford University Medical Center, Redwood City, CA}

Background/Introduction: Suboptimal pedicle screw placement may cause neurological complications following posterolateral lumbar fusions (PLF). To decrease the risk of these complications, computer-assisted navigation (CAN) and intraoperative neuromonitoring such as electromyography (EMG) are often used during PLF to ensure safe pedicle screw placement. While CAN and EMG can improve the safety of pedicle screw placement, their routine use in PLF remains controversial. No studies have directly compared the risk of neurological complications following pedicle screw placement in PLF with and without CAN or EMG.

Materials/Methods: Retrospective longitudinal analyses were performed using the MarketScan ${ }^{\circledR}$ databases from 2007-2014. Patients undergoing PLF surgery with and without CAN and/or EMG for degenerative lumbar disorders were identified via ICD-9-CM and CPT codes. Exclusion criteria were: age <18yrs, revision surgery, concomitant interbody fusions, spinal deformity surgery, fusion to the thoracic spine, and diagnoses of traumatic injuries, pathologic fractures, malignant neoplasms, congenital musculoskeletal disorders, inflammatory arthritis, or infections were. Differences in demographic trends, rates of neurological injuries, and reoperation for pedicle screw revision/removal within 90 days were assessed among the following groups: CAN only, EMG only, CAN and EMG, and no CAN or EMG.

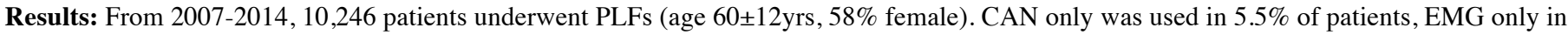
$20 \%$ and CAN and EMG in $0.87 \%$ of patients. Overall, CAN only use increased from $2.7 \%$ in 2007 to $8.72 \%$ in 2014 (p<0.001). There was no difference in the risk of neurological injuries among all groups (CAN only: 2.8\%, EMG only: 3.8\%, CAN and EMG: $2.3 \%$, no CAN or EMG: $3.4 \%$, $\mathrm{p}=0.824)$. In addition, there was no difference in reoperation rates for pedicle screw revision/removal among all groups (CAN only: $0.0 \%$, EMG only: $0.12 \%$, CAN and EMG: $0 \%$, no CAN or EMG: $0.37 \%, p=0.481$ ).

Discussion/Conclusion: In this retrospective review of national administrative data, we found a steady increase in the use of CAN for PLFs from 2007 to 2014. The risk of neurological complications following primary PLFs is low and the routine use of CAN and/or EMG may not decrease this risk.

038. Safety profile of lumbosacropelvic fixation in elderly patients: comparison between S2-alar-iliac screws and iliac screws

Wataru Ishida, $M D^{l}$, Benjamin Elder, $M D, P h D^{l}$, Seba Ramhmdani, MD ${ }^{2}$, Ziya Gokaslan, M.D. ${ }^{3}$, Jean-Paul Wolinsky, M.D. ${ }^{4}$, Nicholas Theodore, $M D^{5}$, Ali Bydon, M.D. ${ }^{4}$, Daniel Sciubba, $M D^{4}$, Timothy Witham, MD ${ }^{6}$, Sheng-Fu Lo, MD

${ }^{1}$ Johns Hopkins University School of Medicine, BALTIMORE, Maryland, ${ }^{2}$, Baltimore, MD, ${ }^{3}$ Brown University, Providence, Rhode Island, ${ }^{4}$ Johns Hopkins Hospital, Baltimore, Maryland, ${ }^{5}$ Johns Hopkins School of Medicine, Baltimore, Maryland, ${ }^{6}$ Johns Hopkins University School of Medicine, Baltimore, $M D$

Background/Introduction: The surgical management of deformity, degenerative pathology, neoplasms, trauma, and infection in the lumbosacropelvic spine remains a challenge due to high mechanical demand around this area, risks of pseudarthrosis, and invasive nature of the procedures. To date, two instrumentation techniques are mainly available: iliac screws (ISs) and S2-alar-iliac (S2AI) screws. Previous articles demonstrated lower rates of complications with S2AI screws as compared to ISs; however, none of them have focused on elderly patients who may harbor significant co-morbidities, and as such, require more meticulous perioperative management, given these invasive, lengthy procedures. Here we report safety profile of S2AI in patients over 60 and address differences between S2AI screws and ISs.

Materials/Methods: Between October 2010, and December 2014, 171 sacropelvic fusion procedures with IS or S2AI screw instrumentation were performed in 161 patients. The inclusion criteria for this study were as follows: (1) patients over 60 years old and (2) patients with more than two-year follow-up periods, which yielded 15 patients (IS) and 41 cases (S2AI), respectively. Rates of complications such as unplanned reoperation and functional outcome measures, such as visual analogue scale for back pain were collected and statistically analyzed.

Results: Baseline characteristics such as age, sex, smoking history, diagnoses, co-morbidities were similar in both groups. The S2AI group had lower rates of reoperation $(19.5 \%$ versus $66.6 \%, \mathrm{p}=0.002)$, surgical site infection $(0 \%$ versus $16.0 \%, \mathrm{p}<0.001)$, wound dehiscence $(0.0 \%$ versus $20.0 \%, \mathrm{p}<0.001)$, and postoperative hyperkalemia $(17.7 \%$ versus $43.8 \%, \mathrm{p}=0.04)$ and had lower volume of estimated blood loss [ml] $(1817.5 \mathrm{versus}$ 2775.6, $\mathrm{p}=0.01$ ) and transfused red blood cell units (4.6 versus 8.0, $\mathrm{p}=0.001)$ than the IS group, while rates of mortality, L5-S1 pseudarthrosis, and other cardiorespiratory complications were similar in both groups. Pain relief and functional recovery were achieved in both groups without any significant intergroup differences. On multivariate analyses, use of S2AI screws over ISs, smoking, and operated levels were independent predictors of volume of transfusion.

Discussion/Conclusion: Use of S2AI screws over ISs in the elderly was associated with lower rates of reoperation, surgical site infection, wound dehiscence, and lower volume of EBL and RBC transfusion, while achieving similar clinical and functional outcomes.

039. Computer-assisted navigation in posterior lumbar fusion increases operative time and length of stay

Nathan Hendrickson, $M D, M S^{l}$, Yubo Gao, $\mathrm{PhD}^{l}$, Cameron Barton, $M D^{1}$, Andrew Pugely, $M D^{2}$

${ }^{l}$ Department of Orthopedics and Rehabilitation, University of Iowa, Iowa City, IA, ${ }^{2}$, New York, NY

Background/Introduction: Computer-assisted navigation in lumbar spine surgery is reported to reduce patient radiation exposure, transpedicular screw insertion errors, and decrease operative time. Adoption rates for navigation in posterior lumbar fusion are unknown. It is important to determine the impact of navigation on patient outcomes and quality metrics in the thirty-day post-operative period. 
Materials/Methods: The American College of Surgeons National Quality Improvement Program database was queried from 2011-2015 to identify patients undergoing elective posterior lumbar spinal fusion (CPT code 22612, 22633, 22630). Cases with clean-contaminated, contaminated or dirty/infected wound class were excluded. Patients were stratified based upon documentation of intraoperative Computer-Assisted Navigation (CAN) (CPT code 61783). Patient age, gender, race, BMI, diabetes mellitus, smoking status, medical comorbidity, resident case involvement and number of segments fused were assessed for potential confounding. We evaluated navigation rates by year to evaluate navigation adoption rates. Propensity score matching was used to reduce patient selection bias for comparison of thirty-day complication rates between navigated and traditional lumbar fusion groups.

Results: There were 25,010 patients in the cohort, with 1,812 (7.2\%) cases involving computer-assisted navigation. Navigation rates increased from $3.4 \%$ in 2011 to $9.6 \%$ in 2014 . Utilization in the most recent study year, 2015, was $7.2 \%$. Short-term complications occurred in 1,973 cases $(8.6 \%)$. After propensity score matching, operative time was increased 14 minutes in the navigated group $(222.0 \mathrm{vs} 205.7 \mathrm{~min}, \mathrm{p}<0.0001)$. Length of stay was longer in the navigation group ( 3.72 vs 3.47 days, $\mathrm{p}=0.0092)$. There was no difference in all cause short-term complications between navigated and non-navigated posterior lumbar spinal fusion cases $(7.17 \%$ vs $7.23 \%, \mathrm{p}=0.9488)$.

Discussion/Conclusion: The results of this study demonstrate steady increase in rates of computer-assisted navigation in posterior lumbar fusion from 2010 to 2014. Computer-assisted navigation resulted in increased operative time and length of hospital stay, but there were no differences in all cause short-term complications between navigated and non-navigated posterior lumbar spinal fusion. Thus, while computer assisted navigation is safe to use in lumbar spine surgery, it is unclear if the increased cost and time associated with this technology provide benefits warranting its use in routine lumbar spine surgery.

\section{Assessing the influence of diabetes and cardiopulmonary disease on patient reported outcomes: pre- and post-operatively} $M D^{5}$

Eli Bunzel, Bachelor of Science ${ }^{1}$, Darrel Brodke, MD ${ }^{2}$, Ashley Neese, B.S. ${ }^{2}$, Yue Zhang, PhD ${ }^{3}$, Jared Reese, Bachelor of Science ${ }^{4}$, Nicholas Spina,

${ }^{l}$ Hamilton College, Salt Lake City, Utah, ${ }^{2}$, Salt Lake City, Utah, ${ }^{3}$ University of Utah, Salt lake City, Utah, ${ }^{4}$ Brigham Young University, Salt Lake City, Utah, ${ }^{5}$ University of Utah, Salt Lake City, Utah

Background/Introduction: Adult Spinal Deformity (ASD) negatively impacts quality of life, yet surgical correction has been associated with complication rates of 30-50\% 1 . To adequately inform patients and manage expectations, we must understand how certain pre-operative factors influence disease presentation and post-operative outcomes. Recently, PROMIS Physical Function (PF) Computer Adaptive Testing (CAT) has gained popularity in measuring the health state of a patient's physical function, and has been validated in the spine population2. This study aims to assess the effect of ASD surgical correction on PF CAT scores in patients with diabetes and cardiopulmonary comorbidities.

Materials/Methods: A retrospective review of all patients undergoing surgical correction of ASD from 2013-Present was performed to identify those who underwent fusion of 4 levels or greater. PF CAT scores were obtained pre- and post-operatively at 3,6 and 12-month time periods. Linear mixed effect models adjusted for age, gender and BMI were performed to compare outcome scores in four cohorts: healthy cohort, diabetes mellitus, COPD and heart disease (history of MI, percutaneous coronary intervention, CHF, previous coronary artery bypass grafting) based on ICD-9 and 10 documentation.

Results: 180 patients met our inclusion criteria. Mean age was $66.8 ; 79(44 \%)$ were male and $101(56 \%)$ were female. Healthy patients improved 6 points from their pre-operative PF CAT score at 12 months post-op, while COPD patients $(\mathrm{n}=10)$ decreased by 1.7 points at 12 months ( $\mathrm{p}=.400)$. At 3 months, patients with Heart Disease $(n=37)$ saw a decrease in PF CAT of 0.8, with an overall improvement trend at 12 months similar to the healthy cohort ( $\mathrm{p}=0.433)$. PF CAT scores for Diabetes Mellitus patients $(\mathrm{n}=51)$ improved more slowly than the healthy cohort from 6 to 12 months $(\mathrm{p}=0.490)$ (Figure 1). Statistical significance was not achieved.

Discussion/Conclusion: We present the largest study, to our knowledge, exploring the responsiveness of PROMIS PF CAT to ASD correction. A trend appears toward an impact of medical comorbidities on these outcome scores. This suggests PF CAT is sensitive to the effect of these disease states on patients' physical function domain, an important consideration for shared decision making on surgical intervention.

\section{Does payer type effect patient satisfaction scores?}

Michael Silverstein, $M D^{l}$, Susan Odum, PhD ${ }^{2}$, Michael Conti Mica, MD ${ }^{3}$, Todd Chapman, Jr., MD ${ }^{4}$, Bruce Darden, MD ${ }^{l}$, Eric Laxer, MD Alden Milam, $M D^{3}$, Alfred Rhyne, $M D^{5}$, P. Brad Segebarth, $M D^{l}$, Leo Spector, $M D^{l}$

${ }^{1}$ OrthoCarolina, Charlotte, $\mathrm{NC},{ }^{2}$ OrthoCarolina Research Institute, Charlotte, $\mathrm{NC},{ }^{3}$, Charlotte, NC, ${ }^{4}$ Washington University School of Medicine, St. Louis, MO, ${ }^{5}$ OrthoCarolina, Charlotte, North Carolina

Background/Introduction: Patient reported outcomes (PROs) have been traditionally collected for outcomes research. They are becoming increasingly important to how providers are being measured and reimbursed by payers and chosen by patients. PROs can help providers improve patient experience, increase referrals and reduce lawsuits. However concerns have been raised regarding the effect of payer type on patient satisfaction. The purpose of this study was to determine if payer type is associated with patient satisfaction.

Materials/Methods: At a large private orthopedic practice, patient satisfaction scores were routinely collected at all visits. Between December 2015 and December 2016, 2,768 patients presenting to our sub-specialty spine center completed a patient satisfaction survey. Patient age, sex, payer type (Medicaid, Medicare, Commercial and Workers Compensation) and satisfaction was queried from our institution's administrative database. Patient satisfaction was measured using the single question: "What is the likelihood that you would recommend this provider to your family and friends?" Patients rated their likelihood to recommend (L2R) using a numeric rating scale from $0-10$ with 10 being the most likely to recommend. 
Mean scores, Net Promoter (NP) category and NP score were analyzed. NP Methodology defines categories (scores 0-6 as detractors; 7-8 as neutral; and 9-10 as promoters) and NP score as the number of detractors subtracted from the number of promoters divided by total number of respondents as a percentage. Wilcoxon Tests and Chi-Square Tests were used to determine differences between L2R and payer type.

Results: There were significant differences $(\mathrm{p}<.0001)$ in the likelihood to recommend scores between the different payer types and in the proportion of net promoters. Medicaid patients reported the highest average and NP scores (9.8 and 96.5) while workers compensation patients reported the lowest (9.1 and 74.8). Of the 2,764 patients with complete survey data, 143 were detractors, 2407 were promoters and 214 patients were neutral. See Table.

Discussion/Conclusion: Payer type does effect patient satisfaction scores. Medicaid patients had the highest average and NP scores while Workers Compensation had the lowest average and NP scores. This information can help inform providers about the effect of payer type on patient satisfaction as measured by Likelihood to Recommend.

\section{The PROMIS® of predictors for improvement in spine fusion surgery}

$\underline{\text { Amy Cizik, } P h D, M P H^{1}}$, Danielle Lavallee, $P h D, P h a r m D^{2}$, Jeffrey Jarvik, $M D, M P H^{2}$, Richard Deyo, MD, MPH ${ }^{3}$, Jesse Fann, $M D, M P H^{2}$, David Flum, $M D, M P H, F A C S^{2}$ OR

${ }^{1}$ University of Washington, Seattle, WA, ${ }^{2}$ University of Washington, Seattle, Washington, ${ }^{3}$ Oregon Health and Science University, Portland,

Background/Introduction: More than 800,000 Americans undergo spine surgery yearly. Several randomized controlled trials and case series report the potential benefits of these procedures, but significant variation in treatment effect after spine surgery has been reported as measured by patient-reported outcomes (PROs). Biopsychosocial factors are thought to be strong predictors of successful outcomes yet such information is not routinely captured in practice. We report results of a study to determine the association between patient-reported biopsychosocial characteristics at baseline and evaluate their association with improvement in PRO function at 30 - 90 days following surgery.

Materials/Methods: We conducted an observational, prospective cohort study within an existing statewide collaborative of spine surgeons. Participants completed a battery of biopsychosocial PRO measures, including PROMIS domains, preoperatively (baseline), 30 - 90 days, and 1 year. Improvement was defined as change in the Oswestry Disability Index (ODI) from baseline to 30-90 days following surgery as: 1) absolute 15 point change, 2) $15 \%$ change, and 3) $\leq 22$ point threshold.

Results: We enrolled 156 adult patients undergoing lumbar fusion surgery. The average age of participants was 62 years (SD 13) and 89 (57\%) identified as female. The baseline mean ODI score was 42 (SD 16; range 8 to 82). Among 129 patients (82.7\%) included in this analysis with 30-90 day ODI scores: $43 \%$ achieved improvement as defined by ODI absolute difference (ODI_AD), 66\% achieved improvement by ODI 15\% change (ODI_PC), and 37\% achieved an ODI patient acceptable symptom state (ODI_PASS). Based on an ODI_AD, patients who improved had higher baseline instrumental support, emotional support, pain interference, NRS back pain, and ODI than those who did not improve following surgery (Table 1).

Discussion/Conclusion: Despite existing evidence suggesting approximately a $66 \%$ response rate or improvement following spine surgery, this number may in fact be lower. Rates in this study suggest lower than $45 \%$ improvement at an early timepoint depending on the definition of improvement. This study suggests that by understanding a patient's baseline biopsychosocial characteristics we may better be able to predict a patient's outcome following spine surgery. These findings also identify emotional support and managing pain as potential targets for intervention.

Poster 01. Potential of human NP-like cells derived from umbilical cord to treat degenerative disc disease: novel mechanism for disk regeneration

Mick Perez-Cruet, $M D^{1}$, Rasul Chaudhry, Prof. Oakland university ${ }^{2}$,Esam Elkhatib, MD $^{3}$, Christina McKee, Graduate Student ${ }^{4}$

${ }^{1}$, Royal Oak, Michigan, ${ }^{2}$, Rochester, MI, ${ }^{3}$, Royal Oak, MI,${ }^{4}$, Rochester, MI

Background/Introduction: Degenerative disc disease (DDD) is a common spinal disorder that manifests with lower back pain. The degeneration of intervertebral disc (IVD) is characterized by the loss of extracellular matrix and dehydration of the nucleus pulposus (NP) of IVDs. Currently, there is no biological treatment to cure this debilitating ailment.

Materials/Methods: In this study, we investigated the efficacy of NP-like cells (NPCs) derived from the umbilical cord (UC) MSCs in restoring degenerated IVDs using a rabbit DDD model. UC -MSCs were induced to differentiate into NPCs by using differentiation medium (DM) for two weeks, labeled with PKH26 and then injected into the degenerated IVDs.

Results: Eight weeks posttransplantation analysis showed that structure and cellularity of the NP improved only in the IVDs that received NPCs. Transplanted IVDs also had higher sGAG and water content compared to the sham and degenerated IVDs. The transplanted cells survived, integrated, and dispersed in the damaged areas of the NP and were functionally active as they expressed human genes, SOX9, ACAN, COL2, FOXF1, KRT19, PAX6, CA12 and COMP as well as human proteins, SOX9, ACAN, COL2 and FOXF1 implicated in NP biosynthesis.

Discussion/Conclusion: These results suggest that NPCs were capable of homing to regenerate NP. The molecular mechanism for NP regeneration was proposed to be regulated via the TGFß1 pathway. This study for the first time demonstrates the feasibility and efficacy of human NPCs derived from UCMSCs to regenerate NP in a rabbit model. These findings should spur interest for clinical studies to treat DDD using NPCs. fusion

Poster 02. Risk factors for perioperative and postoperative complications after minimally invasive transforaminal lumbar interbody 


\section{${ }^{1}$ Rush University Medical Center, Chicago, Illinois, ${ }^{2}$, Chicago, IL,${ }^{3}$ Rush University Medical Center, Chicago, IL}

Background/Introduction: Minimally invasive transforaminal lumbar interbody fusion (MIS TLIF) is becoming an increasingly popular technique for the treatment of lumbar degenerative pathologies. The prevention of complications is necessary in order to avoid poor postoperative outcomes and increased healthcare costs. Many previous investigations have identified risk factors for complications after various spine procedures, but no such study exists in a population solely undergoing MIS TLIF surgeries. The objective of this study is to determine patient risk factors for the development of perioperative and postoperative complications up to 2 years after a primary, single-level MIS TLIF.

Materials/Methods: A prospectively maintained surgical registry of patients was retrospectively reviewed to identify those who underwent primary, single-level MIS TLIF from 2007-2016. The incidence of both medical and surgical complications up to 2 years postoperatively was determined for each patient. Patients were categorized according to demographic, comorbidity, and procedural characteristics. Bivariate Poisson regression with robust error variance was used to determine if an association existed between demographic, comorbidity, or procedural characteristics and complication incidence. A final multivariate model including all demographic, comorbidity, and procedural characteristics as controls was created using backwards, stepwise regression until only those variables with $\mathrm{p}<0.05$ remained.

Results: 390 patients were included in this study. Upon bivariate analysis, age $>50$ years, diabetes mellitus, and operative duration $>105$ minutes were associated with increased medical complication rates after MIS TLIF. Regarding surgical complications, age $\leq 50$ years, obesity, and diabetes mellitus were identified as risk factors on bivariate analysis. Upon final multivariate analysis, operative time $>105$ minutes and diabetes mellitus were independent risk factors for medical complications. Independent risk factors for surgical complications on multivariate analysis included age $\leq 50$ years and hypertension.

Discussion/Conclusion: Older patients and diabetics were at increased risk of medical complications, while younger patients and those with hypertension were at increased risk of surgical complications up to 2-years after MIS TLIF. Practitioners can use this information to identify patients who require preventative care before their procedure or increased postoperative vigilance and clinical monitoring after MIS TLIF

Poster 03. Pre-operative chronic opioid therapy: a risk factor for complications, readmissions, continued opioid use and increased costs after one and two level posterior lumbar fusion

Nikhil Jain, $M D^{I}$, Frank Phillips, $M D^{2}$, Tristan Weaver, $M D^{3}$, Safdar Khan, $M D^{4}$

\section{${ }^{l}$ Ohio State University, Columbus, Ohio, ${ }^{2}$, Chicago, IL, ${ }^{3}$ The Ohio State University, Columbus, Ohio, ${ }^{4}$, Columbus, OH}

Background/Introduction: Risk factors, complications, readmissions, adverse events, and costs associated with chronic opioid therapy (COT) in patients undergoing lumbar fusion are not entirely known. Our objective was to study patient profile associated with pre-operative COT, and study COT as a risk factor for 90-day complications, emergency department (ED) visits, and readmission after primary 1-2 level posterior lumbar fusion (PLF) for degenerative disease of the spine. We also evaluated associated costs as well as risk factors and adverse events related to long term postoperative opioid use.

Materials/Methods: Commercial insurance data from 2007 to Q3-2015 was used to study pre-operative opioid use in patients undergoing primary 1-2 level PLF. 90-day complications, ED visits, readmissions, one-year adverse events, and associated costs have been described. Multiplevariable regression analyses were done to study pre-operative COT patient profile, and study opioid use as a risk factor for complications and adverse events.

Results: 24,610 patients with a mean age of $65.6 \pm 11.5$ years were included. 5,500 (22.3\%) patients had documented opioid use for $>6$ months before surgery. On multiple-variable logistic regression analysis, patients who had pre-operative COT were more likely to be associated with diagnosis of anxiety (OR 1.29, 95\% CI:1.19-1.40), depression (OR 1.15, 95\%CI:1.06-1.25), inflammatory arthritis (OR 1.14, 95\% CI:1.06-1.23), tobacco use disorder (OR 1.50, 95\%CI:1.37-1.64), and drug abuse/dependence (OR 1.43, 95\% CI:1.23-1.67). After adjusting for various demographic and clinical covariates, pre-operative COT was found to be a risk factor for 90-day wound complications (OR 1.19, 95\%CI:1.05-1.35), pain diagnoses (OR 1.10, 95\% CI:1.02-1.19), ED visits (OR 1.31, 95\%CI:1.15-1.49), readmission (OR 1.80, 95\%CI:1.24-2.57), and continued use postoperatively (OR 8.08, 95\% CI:7.40-8.80). Post spinal fusion long-term opioid users had an increased utilization of lumbo-sacral epidural and facet joint injections (OR 2.24, 95\% CI:2.04-2.46), higher risk for revision fusion (OR 1.33, 95\%CI:1.14-1.55), and increased incidence of new onset constipation (OR 1.15, 95\% CI:1.05-1.25) within one-year after surgery. The cost associated with increase resource use in these patients have been reported.

Discussion/Conclusion: The increased risk of complications, readmission, and adverse events with resultant higher resource use in chronic opioid users results in additional healthcare costs, and will have financial implications for all providers in a bundled payment model.

Poster 04. Obese patients may experience worse back pain and less improvement in back pain compared to non-obese patients following lumbar decompression-only surgery

Philip Louie, $M D^{l}$, Bryce Basques, $M D^{l}$, Michael Nolte, $M D^{2}$, Arya Varthi, MD ${ }^{3}$, Steven Heidt, BS ${ }^{2}$, Jeremy Mormol, BS ${ }^{1}$, Justin Paul, MD, $P h D^{4}$, Edward Goldberg, $M D^{5}$, Howard An, $M D^{1}$

${ }^{1}$ Rush University Medical Center, Chicago, IL, ${ }^{2}$ Rush University Medical Center, Chicago, Illinois, ${ }^{3}$ Yale University , New Haven, CT, ${ }^{4}$, New York, $N Y,{ }^{5}$, Chicago, IL 
Background/Introduction: Obesity is a significant comorbidity that can increase the risk, technical difficulty, and outcomes following spine surgery. The impact of obesity on longer-term outcomes following lumbar decompression is unclear. The goal of this study was to compare obese and non-obese patients in terms of patient reported outcomes pre-operatively and at final follow-up between.

Materials/Methods: We performed a retrospective cohort analysis of patients who underwent a primary lumbar laminectomy, discectomy, or a combination of the two. Patients were considered obese if their BMI was 30 or greater. Patient reported outcomes were obtained in the form of Oswestry Disability Index (ODI) scores, Visual Analog Scales (VAS) scores for the back and leg, 12-Item Short Form Mental and Physical Survey (SF-12) scores, and the Veterans Rand 12-Item Health Mental and Physical Survey (VR-12) scores.

Results: Overall, 325 consecutive patients who fulfilled our inclusion criteria were assessed. Average follow-up was 24.14 months (range 6-78 months). There were 200 patients with a BMI less than 30 and 125 patients with a BMI of 30 or greater (obese group). The obese group contained significantly more patients with a diabetes diagnosis (62.6\% vs. 30.7\%; $<<0.001)$ and a higher number of patients with an American Society of Anesthesiologists Physical Status Score (ASA) greater than or equal to 3 [35.2\% vs. 19.0\%; p<0.001]. On multivariate analysis, obese patients had significantly higher VAS-back scores at final follow-up compared to their non-obese counterparts $(4.6 \pm 2.9$ vs $2.6 \pm 2.6 ; p=0.009)$ and significantly less improvement in VAS-back scores ( $3.3 \pm 3.8$ vs $4.5 \pm 3.5$; $\mathrm{p}=0.046)$. Otherwise, there were no significant difference among all pre-operative, post-operative, and the change patient reported outcome scores between the two groups. Re-operation rates were not significantly different ( $7.6 \%$ vs. $11.5 \% ; \mathrm{p}=0.573)$.

Discussion/Conclusion: At approximately 2 years following lumbar decompression-only surgery, obese patients may expect worse postoperative back pain and less improvement in back pain compared to their non-obese counterparts. Otherwise, other patient reported outcomes appear to reveal similar clinical improvement in mental and physical health. These concerns and the effect of obesity on overall health continue to emphasize importance of weight discussions and expectations prior to surgery.

Poster 05. Percent hemoglobin loss is associated with higher morbidity in spine surgery patients

Taylor Purvis, $B A^{1}$; Zach Pennington, B.S. ${ }^{2}$, C. Rory Goodwin, MD, PhD ${ }^{3}$, Camilo Molina, MD ${ }^{4}$, Steven Frank, MD ${ }^{4}$, Daniel Sciubba, MD

${ }^{1}$ Johns Hopkins School of Medicine, Baltimore, MD, ${ }^{2}$ Johns Hopkins University, Baltimore, MD, ${ }^{3}$, Durham, NC, ${ }^{4}$, Baltimore, MD, ${ }^{5}$ Johns Hopkins Hospital, Baltimore, Maryland

Background/Introduction: Several studies in gastrointestinal and cardiac surgical fields have demonstrated the importance of considering delta hemoglobin $(\Delta \mathrm{Hb})$ in the formula for appropriate perioperative blood transfusions, where $\Delta \mathrm{Hb}$ is the difference between preoperative Hb and nadir $\mathrm{Hb}$ concentration during a hospital stay. In this study, we sought to determine the perioperative outcomes associated with percent $\Delta \mathrm{Hb}$ as an independent factor among spine surgery patients.

Materials/Methods: Patients who underwent spine surgery at our institution from 2008-2015 were eligible for this retrospective study. Patients undergoing the following procedures were included: atlantoaxial fusion, subaxial anterior cervical fusion, subaxial posterior cervical fusion, anterior lumbar fusion, posterior lumbar fusion, lateral lumbar fusion, excision of intervertebral disc, and excision of spinal cord lesion. Data on intraoperative transfusion were obtained from an automated prospectively collected anesthesia data management system. Data on postoperative hospital transfusions were obtained through a Web-based intelligence portal. Percent $\Delta \mathrm{Hb}$ was defined as: ((first $\mathrm{Hb}-\mathrm{nadir} \mathrm{Hb}) / \mathrm{first} \mathrm{Hb}) \mathrm{x} 100$. Clinical outcomes included in-hospital morbidity and length of stay associated with percent $\Delta \mathrm{Hb}$.

Results: A total of 3,949 patients who underwent spine surgery were identified. A total of 1204 patients $(30.5 \%)$ received at least one unit of packed red blood cells (PRBC). The median nadir Hb level was $10.6 \mathrm{~g} / \mathrm{dL}$ (IQR, 8.7-12.4 g/dL), yielding a mean percent $\Delta \mathrm{Hb}$ of $23.6 \%$ (SD=15.4\%). Perioperative complications occurred in 234 patients $(5.9 \%)$ and were more common in patients with a larger percent $\Delta \mathrm{Hb}(\mathrm{P}=0.017)$. Hospitalrelated infection, which occurred in 60 patients $(1.5 \%)$, was also more common in patients with a greater percent $\Delta \mathrm{Hb}(\mathrm{P}=0.001)$.

Discussion/Conclusion: Percent $\Delta \mathrm{Hb}$ is independently associated with a higher risk of developing any one perioperative complication and hospital-related infections. Our results suggest that percent $\Delta \mathrm{Hb}$ may be a useful measure to identify patients at risk for adverse perioperative events.

\title{
Poster 06. Patient perceptions of minimally invasive versus open spine surgery
}

\author{
Brittany Haws, $B S^{l}$, Benjamin Khechen, $B A^{1}$, Ankur Narain, $B A^{2}$, Fady Hijji, MD ${ }^{2}$, Gabriel Duhancioglu, MS ${ }^{3}$, Blaine Manning, BS ${ }^{3}$, Matthew \\ Colman, $M D^{4}$, Kern Singh, $M D^{3}$
}

IL

${ }^{1}$ Rush University Medical Center, Chicago, Illinois, ${ }^{2}$, Chicago, IL, ${ }^{3}$ Rush University Medical Center, Chicago, IL, ${ }^{4}$ Rush University, Chicago,

Background/Introduction: MIS surgery has increased in popularity due to proposed advantages in the perioperative and immediate postoperative periods. However, patient preferences and understanding regarding the differences between MIS and open surgery have not been elucidated. Therefore, the aim of this study is to describe patient perceptions of minimally invasive (MIS) versus open surgery, and to determine which factors are most heavily considered by the patient when choosing between approaches.

Materials/Methods: An anonymous questionnaire consisting of 30 questions was administered to patients scheduled to see either an MIS surgeon or an open spine surgeon for a clinical evaluation. 6 questions asked about patient demographics and medical history. 9 questions asked respondents to rate the importance of several criteria when deciding between MIS and open surgery. 15 multiple choice and free response questions asked respondents about their perceptions of MIS versus open surgery in regards to surgical and physician characteristics. Subgroup comparison was 
performed for survey responses between patients seeing a MIS surgeon and patients seeing an open spine surgeon. Statistical analysis used to compare patient responses consisted of linear regression adjusted for demographic differences and chi-square analysis or Fisher's exact test for continuous and categorical variables, respectively.

Results: 326 patients completed the survey. The three most important criteria for patients when choosing between open and MIS surgery were: long-term outcomes, surgeon's recommendation, and complication risk for both MIS and open subgroups. When compared to MIS surgery, the majority of patients perceived open surgery to be more painful (83.8\%), have increased complication risk $(78.5 \%)$, recovery time $(89.3 \%)$, costs $(68.1 \%)$ and require heavier sedation $(62.6 \%)$. If required to have spine surgery in the future, the majority of both patient groups would prefer a minimally invasive approach $(80.0 \%)$.

Discussion/Conclusion: Long-term outcomes, surgeon's recommendation, and complication risk were the most important criteria identified by patients when choosing between open and MIS surgery. Patients also perceived MIS surgery to have advantages over open surgery in regards to postoperative pain, complication risk, recovery time, cost, and anesthesia requirement. Most patients, regardless of their surgeon's preferred technique, appear to prefer a minimally invasive approach to their treatment.

Poster 07. The impact of diabetes mellitus on length of stay and direct hospital costs after minimally invasive transforaminal lumbar interbody fusion

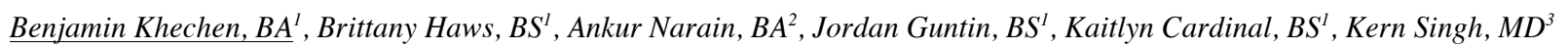

${ }^{l}$ Rush University Medical Center, Chicago, Illinois, ${ }^{2}$, Chicago, IL,${ }^{3}$ Rush University Medical Center, Chicago, IL

Background/Introduction: In spine literature, the presence of diabetes as a comorbidity has been associated with an increased risk for acute postoperative complications, increased length of inpatient stay, and increased costs. However, the majority of these investigations have been performed in study populations undergoing heterogeneous mixtures of procedure types. Very few studies have investigated the effect of diabetes on inpatient length of stay or direct hospital costs in procedure-specific populations. As such, the purpose of this study is to determine if the presence of diabetes mellitus as a comorbidity is associated with inpatient length of stay or direct hospital costs after minimally invasive transforaminal lumbar interbody fusion (MIS TLIF).

Materials/Methods: A prospectively-maintained surgical registry of patients undergoing primary, single-level MIS TLIF for degenerative pathology between 2008-2016 was retrospectively reviewed. Diabetic and non-diabetic patients were propensity matched in a 1:1 fashion for age, gender, and comorbidity burden. An association between diabetic status and preoperative demographic or perioperative variables, including inpatient length of stay, was tested for using student's t-test or chi-square analysis for continuous or categorical variables, respectively. Multivariate linear regression was used to test for an association between diabetic status and direct hospital costs.

Results: 100 patients were included in this analysis. There were no significant differences in age, sex, body mass index, smoking status, or Charlson Comorbidity Index between propensity-matched patients with and without diabetes. In regards to length of stay, no significant differences existed between diabetic and non-diabetic groups. No other significant differences existed in other perioperative variables including operative time, intraoperative blood loss, or complication rate. Multivariate analysis indicated that diabetic status was not associated with differences in total direct hospital costs or cost subcategories after MIS TLIF.

Discussion/Conclusion: Diabetes mellitus was not associated with increased length of stay or direct hospital costs after single-level MIS TLIF. The reduced extent of operative exposure and tissue trauma in MIS TLIF may mitigate the risk of complications in diabetic patients, possibly preventing extensions in hospital stay length. Within the topic of spine surgery, further investigation of the effect of diabetes mellitus on perioperative and postoperative outcomes is required for procedure-specific cohorts.

Poster 08. Comparison of Patient Reported Outcome Measurement Information System (PROMIS) with ODI and VAS in patients with back pain

Themistocles Protopsaltis, $M D^{I}$, Thomas Errico, $M D^{2}$, Aaron Buckland, $M D^{I}$, Charla Fischer, $M D^{3}$, Jared Tishelman, BA ${ }^{2}$, Raj Karia, $M S^{2}$, David S. Jevotovsky, $B A^{2}$

${ }^{1}$ Department of Orthopedic Surgery, NYU Langone Orthopedic Hospital, NYU Langone Health, Manhattan, NY, ${ }^{2}$ Department of Orthopedic Surgery, NYU Langone Orthopedic Hospital, NYU Langone Health, New York, New York, ${ }^{3}$ Columbia University Department of Orthopaedic Surgery, New York City, New York

Background/Introduction: The Patient Reported Outcomes Measurement Information System (PROMIS) Physical Function, PROMIS Pain Intensity, and PROMIS Pain Interference are three instruments that have become increasingly popular for their computer adaptive testing (CAT) methodology. Legacy questionnaires, including ODI and VAS, are limited by the confounding effect of pain and dysfunction in spine regions adjacent to the lumbar spine. This study aims to validate the association of PROMIS and legacy questionnaires and examine the sensitivity of PROMIS CAT to assessing disability in adjacent spine regions.

Materials/Methods: A retrospective analysis was performed on a database of PROMs at a single-institution from 12/2016 to 4/2017. Inclusion criteria were age $>18$ years old and a primary complaint of back pain. The ODI, VAS Back, VAS Neck, VAS Leg, PROMIS Physical Function, PROMIS Pain Intensity, and PROMIS Pain Interference questionnaires were administered. Propensity score matching was employed to compare patients with high and low neck and leg pain, respectively, while controlling for back pain. Respective pain groups were dichotomized by VAS neck and VAS leg ( $\geq 5$ vs $<5$ ). Bivariate correlations were performed to assess the relationships between PROMIS and legacy questionnaires. Independent $\mathrm{t}$-tests were compared between high and low neck and leg pain groups. Statistical significance was $\mathrm{p}<0.05$. 
Results: 484 patients were included in this study. ODI was shown to correlate strongly to PROMIS Physical Function ( $\mathrm{r}=0.749$, $\mathrm{p}<0.001)$, PROMIS Pain Intensity ( $r=0.709, p<0.001)$, and PROMIS Pain Interference $(r=0.790, p<0.001)$. When outcome measures were compared between VAS neck pain groups, there were no significant differences in PROMIS Physical Function, PROMIS Pain Intensity and PROMIS Pain Interference (all $\mathrm{p}>0.05$ ). Patients with high leg pain were more disabled in terms of ODI (45.77 vs. 35.60, $\mathrm{p}<0.001)$. Additionally, there were worse PROMIS Physical Function (36.82 vs 39.09, p=0.017), PROMIS Pain Intensity (53.48 vs 55.55, p=0.011) and PROMIS Pain Interference (62.65 vs 65.66, $\mathrm{p}<0.001)$ scores between the high and low leg pain groups.

Discussion/Conclusion: PROMIS domains demonstrated strong correlations to traditional questionnaires. When controlling for back pain, all PROMIS instruments performed similarly when comparing high and low neck pain. All three PROMIS instruments were sensitive to differences in concurrent leg pain.

Poster 09. Workers' compensation status is predictive of patient reported outcomes following minimally invasive transforaminal lumbar interbody fusion

Brittany Haws, $B S^{1}$, Benjamin Khechen, $B A^{l}$, Jordan Guntin, $B S^{1}$, Kaitlyn Cardinal, $B S^{1}$, Kern Singh, $M D^{2}$

\section{${ }^{l}$ Rush University Medical Center, Chicago, Illinois, ${ }^{2}$ Rush University Medical Center, Chicago, IL}

Background/Introduction: The identification of risk factors for inferior outcomes is of significant importance in the current healthcare climate. Patients that receive Workers' Compensation (WC) benefits have been reported to demonstrate poor surgical outcomes following spine procedures. However, few studies have investigated this relationship among patients undergoing minimally invasive transforaminal lumbar interbody fusion (MIS TLIF). As such, the The purpose of this study is to determine if WC status predicts patient reported outcomes (PROs) following MIS TLIF.

Materials/Methods: A prospectively-maintained surgical database of primary, single-level MIS TLIF patients from 2014-2017 was retrospectively reviewed. PRO measures, including Oswestry Disability Index (ODI) and Visual Analog Scale (VAS) back and leg pain scores were recorded at preoperative and 6-week, 12-week, and 6-month postoperative visits. Rates of minimum clinically important difference (MCID) achievement were determined for ODI and VAS back and leg pain at 6-months postoperative. WC status was tested for an association with demographic and procedural characteristics using student's t-test or chi-square analysis for continuous and categorical variables, respectively. The association between WC status and PROs was assessed using multivariate linear regression controlling for preoperative characteristics.

Results: A total of 204 patients with complete PRO data were included in this analysis. $30 \%(\mathrm{~N}=62)$ of patients had WC benefits, while $70 \%$ $(\mathrm{N}=142)$ did not. Patients with WC were younger, more likely to be male, more likely to smoke, and demonstrated higher rates of obesity (p<0.05 for each). No differences in procedural characteristics, including operative time, estimated blood loss, or length of hospital stay, were demonstrated between WC and non-WC patients. WC patients reported higher preoperative ODI scores and experienced less postoperative improvement ( $<<0.05$ for each). WC patients also reported significantly less postoperative improvement in VAS back and leg pain scores. Finally, patients with WC achieved MCID for ODI and VAS back pain at lower rates than non-WC patients.

Discussion/Conclusion: Patients with WC benefits are younger and are more likely to be male, smoke, and be obese. Additionally, WC patients demonstrate significantly less improvement in PROs following primary, single-level MIS TLIF. As such, patients receiving WC benefits should be appropriately counseled regarding their increased risk for poor surgical outcomes.

\section{Poster 10. Risk factors for a long hospital stay following minimally invasive lumbar discectomy}

Benjamin Khechen, $B A^{1}$, Brittany Haws, $B S^{l}$, Ankur Narain, $B A^{2}$, Fady Hijji, $M D^{2}$, Kaitlyn Cardinal, BS ${ }^{1}$, Jordan Guntin, BS ${ }^{l}$, Kern Singh, $M D^{3}$

\section{${ }^{l}$ Rush University Medical Center, Chicago, Illinois, ${ }^{2}$, Chicago, IL,${ }^{3}$ Rush University Medical Center, Chicago, IL}

Background/Introduction: Minimally invasive lumbar discectomy (MIS LD) has proven to be an effective treatment modality for low back pain and radiculopathy associated with intervertebral disc herniations. With the increasing focus on cost reduction and value-based care, minimization of postoperative length of stay has become an important topic for physicians and hospital administrators. Despite this, risk factors for prolonged length of stay (LOS) after MIS LD have not been previously described. The objective of this study is to determine risk factors for discharge after postoperative (POD) 0 in patients undergoing 1-level MIS LD.

Materials/Methods: A prospectively maintained surgical database of patients who underwent 1-level MIS LD by a single surgeon from 20112016 was reviewed. Long length of stay was defined as discharge after POD 0. Bivariate and stepwise multivariate Poisson regression with robust error variance was used to determine risk factors for discharge after POD 0. Variables analyzed included patient demographics, comorbidities, operative characteristics, preoperative pain scores, postoperative inpatient pain scores, and postoperative narcotics consumption as measured by oral morphine equivalents (OMEs). A p-value $<0.05$ was set as the limit for statistical significance.

Results: A total of 176 patients were included in this analysis. 9.7\% of included patients were discharged on POD 1 or later, qualifying as having a long length of stay. On bivariate analysis, diabetic status $(57.1 \%$ vs. $7.7 \%$, Relative Risk $[R R]=7.43$, p $<0.01)$ and narcotic consumption $<6.00$ $\mathrm{OME} / \mathrm{hr}(13.1 \%$ vs. $1.2 \%, \mathrm{RR}=11.11, \mathrm{p}=0.019)$ were associated with a prolonged length of stay. On stepwise multivariate analysis diabetic status $(\mathrm{RR}=10.5,95 \%$ Confidence Interval $=3.60-30.98, \mathrm{p}<0.001)$ was found to be independently associated with a prolonged length of stay after MIS LD.

Discussion/Conclusion: The results of this study indicate that diabetic status is an independent risk factor for increased LOS following singlelevel MIS LD. Delayed hospital discharge can lead to increased costs, increased risk of complications, and decreased patient satisfaction. As such, providers can use this information to better counsel diabetic patients and monitor these patients more closely following MIS LD. Additional work must be done to better understand risk factors for increased LOS following MIS LD in procedure-specific populations. 
Poster 11. Postoperative outcomes based on American Society of Anesthesiologists score after minimally invasive transforaminal lumbar interbody fusion

Benjamin Khechen, $B A^{1}$, Brittany Haws, $B S^{1}$, Ankur Narain, $B A^{2}, F a d y$ Hijji, $M D^{2}$, Jordan Guntin, $B S^{1}$, Kaitlyn Cardinal, $B S^{1}$, Kern Singh, $M D^{3}$

${ }^{1}$ Rush University Medical Center, Chicago, Illinois, ${ }^{2}$, Chicago, IL, ${ }^{3}$ Rush University Medical Center, Chicago, IL

Background/Introduction: Minimally invasive transforaminal lumbar interbody fusion (MIS TLIF) is an effective treatment for lumbar degenerative disorders. ASA score has previously been associated with major operative complications, increased direct costs, and increased 30-day mortality rates following surgery. However, few studies have analyzed ASA score in a population of patients undergoing minimally invasive spine procedures. This study aims to determine if an association exists between preoperative ASA score and improvements in patient-reported outcomes (PRO) including Oswestry Disability Index (ODI), Visual Analogue Scale (VAS) back and VAS leg pain after MIS TLIF.

Materials/Methods: A prospective surgical database of patients who underwent a primary, single-level MIS TLIF for degenerative pathology between 2010-2016 was reviewed. Patients were excluded if they had incomplete preoperative PRO survey data. Patients were grouped based on ASA score $\leq 2$ and ASA score $>2$. ASA score was tested for association with demographics, procedural characteristics, preoperative ODI, and preoperative Visual Analog Scale (VAS) back and leg pain scores using student's t-test, chi-square analysis, and Fisher's exact test for continuous, categorical, and non-parametric variables, respectively. Postoperative improvement in ODI, VAS leg, and VAS back scores between ASA score cohorts was then compared using linear (continuous) or Poisson regression with robust error variance (categorical) adjusted for patient demographics, preoperative characteristics, and preoperative PRO score.

Results: A total of 113 patients were included in this analysis. 91 patients (80.53\%) had an ASA score $\leq 2$, and $22(19.47 \%)$ had an ASA score $>$ 2. There were no statistically significant differences in demographic characteristics or preoperative ODI, VAS back, and VAS leg score between ASA cohorts $(\mathrm{p}>0.05)$. There were also no statistically significant differences in operative characteristics, preoperative diagnosis, or surgical instrumentation between ASA cohorts ( $\mathrm{p}>0.05)$. ASA cohorts demonstrated no statistically significant differences in PRO improvement at 6-week, 12-week, or 6-month after surgery $(\mathrm{p}>0.05)$.

Discussion/Conclusion: The results of this study suggest that ASA score is not associated with improvements in PRO scores after MIS TLIF. As such, patients with higher comorbidity burden are likely to achieve similar improvements in disability and pain following MIS TLIF compared to those with lower comorbidity burden.

Poster 12. Patients undergoing three level or greater decompression-only surgery for lumbar spinal stenosis have similar outcomes to those undergoing single-level surgery at two years

Philip Louie, $M D^{l}$, Michael Nolte, $M D^{2}$, Bryce Basques, $M D^{1}$, Victor Lei, $B S^{3}$, Tarush Khurana, BS ${ }^{3}$, Justin Paul, $M D$, PhD ${ }^{4}$, Arya Varthi, MD ${ }^{5}$, Edward Goldberg, $M D^{6}$, Howard An, $M D^{l}$

${ }^{l}$ Rush University Medical Center, Chicago, IL, ${ }^{2}$ Rush University Medical Center, Chicago, Illinois, ${ }^{3}$, Chicago, Illinois, ${ }^{4}$, New York, NY, ${ }^{5}$ Yale University, New Haven, CT, ${ }^{6}$, Chicago, IL

Background/Introduction: Stability following multi-level decompressive laminectomy without fusion has been debated using in vitro biomechanical and radiographic models. However, there is a lack of information regarding clinical outcomes for these patients. The goal of this study was to identify the association between clinical outcomes and number of levels decompressed via laminectomy for treatment of lumbar spinal stenosis.

Materials/Methods: We performed a retrospective cohort analysis of patients who underwent a primary lumbar laminectomy between 20092015 by one of two senior orthopaedic spine surgeons for lumbar spinal stenosis. Patients were divided into two groups based on the number of decompression levels: single-level or three or more levels. Patient reported outcomes were obtained in the form of Oswestry Disability Index (ODI) scores, Visual Analog Scales (VAS) scores for the back and leg, 12-Item Short Form Mental and Physical Survey (SF-12) scores, and the Veterans Rand 12-Item Health Mental and Physical Survey (VR-12) scores.

Results: Overall, 138 consecutive patients were assessed, of which 106 underwent a single-level, and 32 underwent a three or more level laminectomy. Average follow-up was 24.2 months. There were no significant differences in the pre-operative VAS-back, VAS-leg or ODI scores between the single-level laminectomy and three or more level laminectomy groups. Both groups of patients experienced significant improvements in these clinical outcomes post-operatively with no clinically significant difference in the degree of improvement. There was no difference in the likelihood for reoperation following three or more levels of decompression (6.45\% vs. $9.43 \%$ for single level, OR=4.46, $\mathrm{P}=0.234)$. Specifically, four single-level patients $(3.8 \%)$ and two of the three or more level $(6.2 \%)$ patients underwent a revision to a posterior spinal fusion at the levels that were originally decompressed at an average of 24 months (single-level 22.1 months; $3+$ levels 25.4 months) after the original surgery.

Discussion/Conclusion: Patients undergoing decompression of three or more levels present with similar post-operative outcomes to those who undergo a single-level decompression for lumbar spinal stenosis. Under specific clinical and radiographic criteria, a multi-level decompression of three or more levels may be a safe and effective procedure with acceptable outcomes at two years after surgery.

Poster 13. Physical function outcomes in patients with cardiopulmonary disease undergoing lumbar spinal fusion

Jared Reese, Bachelor of Science ${ }^{1}$, Darrel Brodke, $M D^{2}$, Ashley Neese, B.S. ${ }^{2}$, Yue Zhang, PhD ${ }^{3}$, Eli Bunzel, Bachelor of Science ${ }^{4}$, Mathieu Squires, B.S. ${ }^{5}$, Nicholas Spina, $M D^{6}$ 


\begin{abstract}
${ }^{1}$ Brigham Young University, Salt Lake City, Utah, ${ }^{2}$, Salt Lake City, Utah, ${ }^{3}$ University of Utah, Salt lake City, Utah, ${ }^{4}$ Hamilton College, Salt Lake City, Utah, ${ }^{5}$ University of Utah School of Medicine, Salt Lake City, UT, ${ }^{6}$ University of Utah, Salt Lake City, Utah
\end{abstract}

Background/Introduction: The PROMIS® Physical Function (PF) Computerized Adaptive Test (CAT) is a questionnaire used to measure a patient's physical function and has been validated in the spine population. However, the responsiveness of PF CAT scores to specific interventions within the lumbar spine and the effects of medical comorbidities on these scores have yet to be investigated. The purpose of this study is to evaluate the responsiveness of PROMIS PF CAT in lumbar fusions and the effects of cardiopulmonary comorbidities on these outcomes.

Materials/Methods: A retrospective chart review identified patients who underwent a single or two level lumbar fusion between October 2013 to June 2017. Patients were then stratified into two cohorts (either cardiac or pulmonary) based on ICD-9 and ICD-10 codes - such as CHF, arrhythmias, MI, PE, and COPD. An adjusted linear regression model was constructed to analyze PF CAT scores pre- and post-operatively at 3 , 6, and 12 month time points.

Results: Of the 972 patients identified (mean age 61,48\% male), 71 had a cardiac comorbidity and 79 had a pulmonary comorbidity. When adjusted for age, gender, and BMI, cardiac patients PF CAT scores ranked lower than non-cardiac patients at the preoperative, 3, 6, and 12 month time points (Cardiac: 42.9, 44.6, 44.9, 45.3; Non-Cardiac: 43.1, 45.5, 47,9, 47.8), with a significant difference found at the 6 month (p=0.016) and 12 month $(\mathrm{p}=0.003)$ time points. Pulmonary patients PF CAT scores ranked lower than non-pulmonary patients across all time points (Pulmonary: 41.0 , 42.4, 41.5, 41.5; Non-pulmonary: 43.2, 45.7, 48.2, 48.1), with a significant difference found at the preoperative ( $\mathrm{p}=0.012), 6 \mathrm{month}(\mathrm{p}=0.002)$, and 12 month $(\mathrm{p}<0.001)$ time points (Figure 1$)$.

Discussion/Conclusion: We present the largest study, to our knowledge, demonstrating the treatment response of PROMIS PF CAT following one and two level lumbar fusion over a one year period of time. Cardiopulmonary comorbidities limit improvement of patients' physical function domain as compared to healthy controls. In this population, adjusting treatment expectations based on PROMIS or assessing different outcomes, such as pain, may improve the evaluation of treatment efficacy.

Poster 14. The effect of preoperative medications on length of stay, inpatient pain, and narcotics consumption following minimally invasive transforaminal lumbar interbody fusion

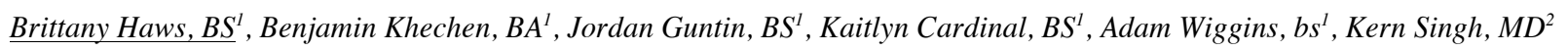

${ }^{1}$ Rush University Medical Center, Chicago, Illinois, ${ }^{2}$ Rush University Medical Center, Chicago, IL

Background/Introduction: Excess postoperative pain can prolong hospital stay or lead to narcotics dependence. Previous studies have identified preoperative comorbidities and narcotic use as risk factors for increased length of hospital stay, inpatient pain, and narcotics consumption. However, little is known regarding the effects of preoperative medications on outcomes following spine surgery. This study aims to determine the association between preoperative medications and length of stay, inpatient pain, and narcotics consumption following a minimally invasive transforaminal lumbar interbody fusion (MIS TLIF).

Materials/Methods: Patients undergoing primary, single-level MIS TLIF were reviewed. Preoperative medications taken within 30 days prior to surgery were recorded for each patient and categorized by medication type. Patient demographic and procedural characteristics were recorded. Patients were categorized based on discharge date, average inpatient Visual Analog Scale (VAS) pain on postoperative day (POD) 0, and narcotics consumption on POD0 as expressed in oral morphine equivalents (OME). Poisson regression with robust error variance controlling for preoperative characteristics was used to determine the association between preoperative medications and length of stay, pain scores, and narcotics consumption. Multivariate analysis was performed using a backwards, stepwise regression to identify independent risk factors for prolonged stay, increased pain, and greater narcotics consumption.

Results: 138 patients were included in this analysis. Benzodiazepines were associated with discharge after POD 2 (Relative Risk [RR] $=2.1,(95 \%$ Confidence Interval $[95 \% \mathrm{CI}]=1.09-4.17, \mathrm{p}=0.026)$. Benzodiazepines $(\mathrm{RR}=5.9,95 \% \mathrm{CI}=2.69-12.81, \mathrm{p}<0.001)$ and preoperative narcotics $(\mathrm{RR}=3.0$, $95 \% \mathrm{CI}=1.12-7.80, \mathrm{p}=0.029)$ were risk factors for pain $\geq 7$ on POD0. On multivariate analysis, benzodiazepines were an independent risk factor for prolonged stay. Benzodiazepines, narcotics, and non-steroidal anti-inflammatories were identified as independent risk factors for increased postoperative pain.

Discussion/Conclusion: The results of this study suggest that benzodiazepines are a risk factor for increased length of stay and postoperative pain following MIS TLIF. Preoperative narcotics were also identified as a risk factor for postoperative pain though this did not lead to increases in narcotics consumption. Patients taking these medications should undergo more vigilant perioperative monitoring for adequate pain management. More work must be done to further elucidate the association between preoperative medications and postoperative outcomes following MIS TLIF.

Poster 15. Patient perceptions of iliac crest bone grafting in minimally invasive transforaminal lumbar interbody fusion

$\underline{\text { Brittany Haws, } B S^{1}}$, Benjamin Khechen, $B A^{1}$, Kaitlyn Cardinal, $B S^{1}$, Jordan Guntin, $B S^{1}$, Amir Siddiqui, $n / a^{1}$, Kern Singh, $M D^{2}$

${ }^{1}$ Rush University Medical Center, Chicago, Illinois, ${ }^{2}$ Rush University Medical Center, Chicago, IL

Background/Introduction: Iliac crest bone grafting (ICBG) is an effective option for promoting arthrodesis in minimally invasive transforaminal lumbar interbody fusion (MIS TLIF). ICBG harvest has been associated with postoperative pain at the donor site, leading to the development of alternative biologics. The true incidence of donor site pain has been debated in the literature due to the varying techniques associated with its harvest. This study aims to determine patient's perception of ICBG harvesting and the development of donor site pain following MIS TLIF. 
Materials/Methods: Patients that underwent primary, single-level MIS TLIF with ICBG were retrospectively identified. ICBG bone graft harvesting was performed using a percutaneous technique with a tubular retractor. Patients that had completed at least three months of postoperative follow-up were included. A survey was administered to assess if patients could accurately describe which side of their iliac crest they believed was harvested, and if they were experiencing any pain perceived to be originating from the donor site.

Results: 79 patients were included in this analysis. 51.9\% of patients underwent MIS TLIF at the level of L4-L5, 44.3\% at L5-S1 and 3.8\% at L3-L4. The majority of patients had the ICBG harvested from the left iliac crest (97.5\%). Approximately half of the patients correctly identified the side of harvest $(50.6 \%)$. Furthermore, $48.1 \%$ of patients reported they were not confident or had guessed on their response. Patients that reported pain from the ICBG were more likely to feel confident or somewhat confident in their harvest site identification (57.9\% vs $46.3 \%)$ but less likely to be correct (36.8\% vs $63.4 \%)$ than patients without pain. 22 patients correctly identified the side of harvest without guessing. Of these, 11 reported experiencing pain.

Discussion/Conclusion: Approximately half of patients undergoing MIS TLIF with ICBG are able to correctly identify which side of their iliac crest was harvested. The majority of patients reporting pain were unable to correctly identify the side of harvest. This suggests that most patients are likely attributing other sources of pain to their ICBG. As such, rates of donor site pain may be over-reported in the current literature with contemporary harvesting techniques.

Poster 17. Validity of PROMIS in minimally invasive transforaminal lumbar interbody fusion: a preliminary evaluation

Brittany Haws, $B S^{l}$, Benjamin Khechen, $B A^{l}$, Kaitlyn Cardinal, $B S^{l}$, Jordan Guntin, $B S^{l}$, Kern Singh, $M D^{2}$

${ }^{1}$ Rush University Medical Center, Chicago, Illinois, ${ }^{2}$ Rush University Medical Center, Chicago, IL

Background/Introduction: Patient-reported outcomes are commonly used to evaluate treatment efficacy. Inefficiencies in standard measurement tools often prove to be a barrier to data collection. The Patient-Reported Outcomes Measurement Information System (PROMIS) was developed to overcome these limitations. This tool implements computer adaptive testing, which allows for assessment of physical function in fewer questions than "static" metrics. Within spine surgery populations, moderate to strong correlations with Oswestry Disability Index (ODI) and Short Form (SF)-36 have been reported for PROMIS. However, data is limited regarding the efficacy of this tool in patients undergoing minimally invasive transforaminal lumbar interbody fusion (MIS TLIF).

Materials/Methods: A prospectively-maintained surgical registry of patients undergoing primary, 1-2 level MIS TLIF was retrospectively reviewed. Patients with incomplete PROMIS data were excluded. Postoperative changes in PROMIS physical function scores at 6-weeks, 12-weeks, and 6-months were analyzed using paired t-tests. PROMIS scores were compared to traditional outcome measures including SF-12 physical function, ODI, and Visual Analog Scale (VAS) back and leg scores. Correlations were tested using Pearson correlation coefficient with strength of association interpreted as follows: $0.1 \leq|r|<0.3$, small; $0.3 \leq|r|<0.5$, moderate; and $|r| \geq 0.5$, large. Statistical significance was set at $p<0.05$.

Results: 74 patients were included in this analysis after exclusion of those without PROMIS scores. The average preoperative PROMIS score was $35.92 \pm 6.98$. There were significant improvements in postoperative PROMIS scores at 12 weeks $(41.33$, $\mathrm{p}=<0.001)$ and 6 months $(43.58$, $\mathrm{p}<0.001)$. PROMIS scores demonstrated significant correlation with SF-12, ODI, and VAS leg scores ( $<<0.05)$. Strong associations with PROMIS scores were observed for SF-12 ( $\mathrm{r}$ range: 0.650 to 0.854 ), ODI ( $\mathrm{r}$ range: -0.525 to -0.831 ), and 6 month VAS back ( $\mathrm{r}=-0.693$ ).

Discussion/Conclusion: Physical function as measured by PROMIS improves significantly at 12 weeks and 6 months following MIS TLIF. Additionally, PROMIS scores demonstrate strong correlations with SF-12 and ODI scores. These results suggest that PROMIS scores may be used as a valid assessment of physical function in MIS TLIF. Further work is required to determine the full benefits of this measure in specific spine populations.

Poster 18. Iliac crest bone graft for minimally invasive transforaminal lumbar interbody fusion: a prospective analysis of inpatient pain, narcotics consumption, and costs

$\underline{\text { Brittany Haws, } B S^{1}}$, Benjamin Khechen, $B A^{1}$, Ankur Narain, $B A^{2}$, Fady Hijji, $M D^{2}$, Kaitlyn Cardinal, BS ${ }^{1}$, Jordan Guntin, BS ${ }^{1}$, Kern Singh, $M D^{3}$

\section{${ }^{l}$ Rush University Medical Center, Chicago, Illinois, ${ }^{2}$, Chicago, IL,${ }^{3}$ Rush University Medical Center, Chicago, IL}

Background/Introduction: Iliac crest bone grafting (ICBG) has been associated with enhanced fusion rates. However, concerns have been raised in regards to increased operative time and post-operative pain. The advantages of ICBG compared to other spinal fusion adjuncts have been debated. However, little is known regarding this technique in the setting of a minimally invasive transforaminal lumbar interbody fusion (MIS TLIF). This study aims to determine if an association between increased acute pain, post-operative time, and direct hospital costs exists between the use of ICBG and a historical control involving Bone Morphogenic Protein (BMP)-2 following a primary, single-level MIS TLIF.

Materials/Methods: Prospective, consecutive analysis of patients undergoing primary, single-level MIS TLIF with ICBG were compared to a historical cohort of consecutive patients that received BMP-2. Operative characteristics were compared between groups using chi square analysis or independent t-test for categorical and continuous variables, respectively. Postoperative inpatient pain was measured using the Visual Analog Scale (VAS), and inpatient narcotics consumption was quantified as oral morphine equivalents (OMEs) for both groups. Outcomes were compared between groups using multivariate regression controlling for preoperative characteristics. Statistical significance was set at $\mathrm{p}<0.05$.

Results: A total of 98 patients were included in this analysis, 49 in each cohort. No significant differences were noted between cohorts except in regards to gender (more males in the BMP cohort, $67.35 \%$ vs $46.94 \%, \mathrm{p}=0.041$ ). There was a significant increase in operative time ( 14.53 minutes, $\mathrm{p}=0.006)$ and estimated blood loss $(16.64 \mathrm{~mL}, \mathrm{p}=0.014)$ in the ICBG cohort. No significant differences were identified for inpatient length of stay or 
VAS scores. Narcotics consumption was similar between groups on postoperative days 0 and 1 . ICBG was associated with decreased total direct costs $(\$ 19,315$ vs $\$ 21,645, \mathrm{p}<0.001)$ as compared to BMP-2.

Discussion/Conclusion: Patients undergoing MIS TLIF that underwent ICBG experienced increases in operative time and estimated blood loss that were not clinically significant. Furthermore, iliac crest harvesting did not result in either an increase in acute pain or narcotics consumption. Further followup is necessary to determine the associated arthrodesis rates and long term outcomes between each cohort.

\section{Poster 19. Effect of vancomycin on postoperative infection rates in thoracic spinal cord stimulation Implants}

Katherine Sullivan, None. ${ }^{1}$, Swamy Kurra, $M B B S^{2}$, Warren Wulff, $M D^{3}$, William Lavelle, $M D^{2}$

${ }^{1}$ Upstate Medical University, Cazenovia, New York, ${ }^{2}$, Syracuse, NY, ${ }^{3}$, Dewitt, New York

Background/Introduction: Spinal cord stimulators (SCS) are used to treat chronic neuropathic pain. Intraoperative vancomycin powder offers protection against surgical site infections (SSI). However, there is a general lack of information regarding patient demographics and surgical procedures that may benefit the most from intraoperative vancomycin powder. The purpose of this study was to analyze rates of infection in patients where prophylactic powdered vancomycin was administered during spinal cord stimulation implanted devices.

Materials/Methods: Retrospectively reviewed 533 consecutive patients from a single academic center who had spinal cord stimulation devices implanted at the thoracic vertebrae level from 2012 through 2017 for chronic neuropathic pain. Patients' demographics, surgical data, postoperative charts and up to 6 months follow-up visits were reviewed. Patients were divided into two groups: Group 1 (n=399) patients who received intraoperative vancomycin powder; and Group $2(n=134)$ patients who did not receive vancomycin powder. ANOVA analyses were performed; $\mathrm{p}<$ 0.05 was considered statistical significant.

Results: The study sample $\mathrm{n}=533$; mean age $=52$ yrs (19-92), gender: $51 \%$ females and $49 \%$ males, mean vancomycin dosage $=0.29(0.25$ to $1 \mathrm{~g})$. $75 \%$ of the patients received perioperative vancomycin powder. Demographics were similar between the groups; however there were more active smokers and higher index (revision) surgery rates in Group 2 and more diabetic patients in Group 1 (Table 1). The overall postoperative infection rate was $3 \%(16)$ with the rate significantly lower in Group $1(n=8,2 \%)$ versus Group $2(n=8,6 \%), p=0.02 .50 \%(n=4)$ of the infections were late infections ( $>3$ month) in Group 2 and none reported in Group 1. All the infections were successfully managed with I\&D, stimulation device removal and IV antibiotics.

Discussion/Conclusion: The overall postoperative infection rate for thoracic spinal cord stimulants was 3\%, and significantly lower in patients receiving vancomycin powder. Addition of vancomycin powder to the two wounds just before closure can decrease the infection rate by two-thirds.

\section{Poster 20. Hyperelastic bone composite scaffolds for de novo bone formation: composition matters}

Ryan Lubbe, $B S^{1}$, Adam Jakus, PhD ${ }^{2}$, Adam Driscoll, $B S^{1}$, Meraaj Haleem, BS ${ }^{1}$, Kevin Chang, BS ${ }^{1}$, Tristan Maerz, Ph.D. ${ }^{3}$, Kevin Baker, Ph.D ${ }^{3}$, Ramille Shah, PhD' ${ }^{2}$, Wellington Hsu, M.D. ${ }^{4}$, Erin Hsu, PhD ${ }^{5}$

${ }^{1}$ Northwestern University Department of Orthopaedic Surgery, Chicago, Illinois, ${ }^{2}$ Northwestern University, Chicago, Illinois, ${ }^{3}$, Royal Oak, Michigan, ${ }^{4}$ Northwestern Memorial Hospital, Chicago, Illinois, ${ }^{5}$ Northwestern University Department of Orthopaedic Surgery, Chicago , IL

Background/Introduction: Bone graft extenders for spine fusion include demineralized bone matrix (DBM) and ceramic-based products, but their efficacy is insufficient to qualify them as bona fide bone graft substitutes (BGS). We previously developed a 3D-printable scaffold composed of synthetic hydroxyapatite particles (HA) and poly lactic-co-glycolic acid (PLGA). With this study, we sought to improve the efficacy of this hyperelastic "bone" composite (HBC) by incorporating osteoinductive DBM particles. We evaluated the ability of various iterations of these HADBM HBC scaffolds to promote bone regeneration and spine fusion in a rat posterolateral spine fusion (PLF) model.

Materials/Methods: Female Sprague Dawley rats underwent L4-L5 PLF utilizing HBC scaffolds composed of 30 vol.\% PLGA and a 70 vol.\% particles. For each group, the particle components consisted of the following vol.\% ratios of HA:DBM: 1:0, 3:1, 1:1, 1:3, and 0:1. Eight weeks postoperative, spine fusion was evaluated via radiography and manual palpation. Synchrotron microCT was used to qualitatively assess bone formation within the struts of the scaffolds. Histology (Hematoxylin/Orange G/Alcian Blue and Masson's Trichrome) was used to characterize the bone growing within/around the struts and the bone-scaffold interface.

Results: 3:1 HA:DBM HBC scaffolds had the highest fusion rate (92\%), although this was only significantly higher than the 0:1 group (42\% fusion; $\mathrm{p}<0.05$ ). The 1:0,1:1, and 1:3 scaffolds achieved fusion rates of $58 \%, 58 \%$, and $67 \%$, respectively. Synchrotron microCT showed that the $1: 3$ scaffolds elicited the greatest amount of de novo bone spicule formation, while the 1:0 scaffold elicited none.

Discussion/Conclusion: This study evaluated the use of 3D printed HBC scaffolds containing both HA and DBM particles as a potential BGS. Varying the proportions of HA:DBM within each scaffold resulted in only slight differences in fusion scores and rates. However, microCT showed that de novo bone spicule formation was only present within scaffold struts that contained DBM particles. We hypothesize that 1) the presence of osteoinductive DBM is key to de novo bone formation, and 2) the hydroxyapatite provides the calcium and phosphate necessary to re-mineralize the DBM particles. Future studies will aim to further improve the efficacy of this HBC as a BGS for spine fusion.

Poster 21. The use of computed tomography (CT) in the evaluation of spondylolisthesis

Michael Danaher, $B S^{1}$, Frank Bailey, $B S^{1}$, Swamy Kurra, $M B B S^{2}$; Katherine Sullivan, None. ${ }^{3}$, Nathaniel Ordway, MS,PE ${ }^{2}$, William Lavelle, MD ${ }^{2}$

${ }^{1}$ Upstate Medical University, Syracuse, New York, ${ }^{2}$, Syracuse, $N Y,{ }^{3}$ Upstate Medical University, Cazenovia, New York 
Background/Introduction: Spondylolisthesis can be qualified using various measurements (pelvic incidence(PI), sacral slope(SS), and pelvic tilt). Pelvic tilt varies based on patient position, but PI does not change with position. PI is usually measured in lateral standing $\mathrm{X}$-rays, however, overlapping of femoral heads or magnification can give false values. Studies report measuring PI in CT scans is more precise. Several studies demonstrated a correlation of PI with spondylolisthesis on radiographs, but limited studies used CT. We used CT to qualify the clinical significance of spondylolisthesis.

Materials/Methods: Retrospectively reviewed patients $(\mathrm{n}=361)$ who had pelvic/abdominal CT scans. Documented: age, average PI (right and left), spondylolisthesis (isthmic or degenerative), spondylolisthesis grading and location, and SS. Using sagittal CT scans PI determined (measured angle between hip axis to orthogonal line originating at center of superior endplate axis of first sacral vertebra) and SS. Average PI calculated using both sides. Patients grouped: spondylolisthesis $(n=45)$ and without spondylolisthesis $(n=316)$. Compared data with age, PI and SS. Sub-groups by location (L4/L5 and L5/S1) and type of spondylolisthesis. ANOVA and Chi-square tests used; $\mathrm{P} \leq 0.05$ considered statistically significant.

Results: Mean age 58 years (range: 21-97); mean age (66 years) with spondylolisthesis (L4/L5 and L5/S1) higher than without spondylolisthesis (mean=57 years), $\mathrm{p}=0.006$. Mean PI and SS with spondylolisthesis (L4/L5 and L5/S1) 59 degrees (range, 34-80) and 100 degrees (range, 85 -115); and without spondylolisthesis 57 degrees (range, 27 -87) and 101degrees (range, 84-115), p=0.29 and p=0.61, respectively. Mean PI not statistically different among isthmic spondylolisthesis(L4/L5) patients (mean=61 degrees, range, 51 -73), degenerative spondylolisthesis $($ L4/L5) $($ mean=56 degrees, range $=37-71$ ), and without spondylolisthesis (mean $=57$ degrees, range $=27-87), p=0.47$. Mean PI significantly higher with isthmic spondylolisthesis(L5/S1) (mean=61 degrees) and lower in degenerative spondylolisthesis(L5/S1) (mean=52 degrees) compared to without spondylolisthesis (mean=57 degrees), $\mathrm{p}=0.06$. Mean PI higher with Grade 2 spondylolisthesis $(\mathrm{L} 5 / \mathrm{S} 1)(\mathrm{mean}=65$ degrees, range $=57-73)$, compared to Grade 1 spondylolisthesis $(\mathrm{L} 5 / \mathrm{S} 1)($ mean=57 degrees, range=34-80) and without spondylolisthesis (mean=57 degrees, range=27 -87$)$, but did not reach statistical significance; $\mathrm{p}=0.18$.

Discussion/Conclusion: CT scans appear reliable for correlating PI to spondylolisthesis. CT scans are more precise than standing lateral x-rays to determine PI. Limitations were retrospective nature and small sample size.

Poster 22. Different fusion approaches for single-level lumbar spondylosis have similar perioperative outcomes

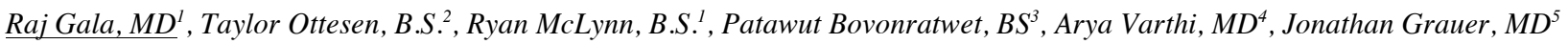

${ }^{l}$ Yale School of Medicine, New Haven, Connecticut, ${ }^{2}$ Yale University School of Medicine, New Haven, CT, ${ }^{3}$ Yale School of Medicine, New Haven, CT, ${ }^{4}$ Yale University, New Haven, CT ${ }^{5}$, New Haven, CT

Background/Introduction: Low back pain from lumbar spondylosis affects a large proportion of the population. The operative treatment remains controversial and includes a variety of surgical procedures, mostly revolving around fusion. Arthrodesis can be performed through posterolateral spinal fusion (PSF), transforaminal lumbar interbody fusion (TLIF), anterior lumbar interbody fusion (ALIF), or combined anteriorposterior fusion (AP fusion). These approaches have comparable long-term outcomes when treating spondylosis, but their possible differences in perioperative complications have not been well studied.

Materials/Methods: Patients with a diagnosis of lumbar spondylosis were reviewed in the American College of Surgeons National Quality Improvement Program (ACS-NSQIP) database, from the years 2010 to 2016, who underwent a single-level lumbar fusion surgery. The NSQIP database tracks perioperative outcomes for thirty days after surgery. The patients were separated into groups based on their procedure (PSF, TLIF, ALIF, or AP fusion). Risk adjusted multivariate regression models accounting for age, BMI, functional status, ASA class, and sex, were run with and without the procedure outcome variable. Complication rates were then compared using nested models to determine likelihood-ratios. Analysis was secondarily done with propensity score matching to confirm findings.

Results: 1816 patients with single-level lumbar spondylosis were identified, of which 322 underwent PSF, 800 underwent TLIF, 460 underwent ALIF, and 234 underwent AP fusion. With the multivariate regression models, there were no differences among the groups in thirty-day complication rates and no differences in the aggregated groupings of any adverse event, serious adverse event, or minor adverse event (Table 1). Secondary analysis with propensity score matching showed no statistically significant differences.

Discussion/Conclusion: Debate continues regarding the optimal surgical procedure for lumbar spondylosis. This study showed that perioperative complications were similar among the surgical approaches for single-level fusion for lumbar spondylosis. These findings suggest that patient/surgeon preference and long-term outcome studies are better used to recommend one surgical approach over another.

Poster 23. Tobacco use and complications following spinal fusion: a comparison of the NSQIP and NIS datasets

Wesley Durand, $S c B^{1}$, J. Mason DePasse, $M D^{2}$, Steven Bokshan, $M^{2}$, Adam Eltorai, $B A^{3}$; Alan Daniels, $M^{4}$

${ }^{1}$ Brown University, Warren Alpert Medical School, Providence, RI, ${ }^{2}$ Department of Orthopaedics, Warren Alpert Medical School of Brown University, Providence, Rhode Island, ${ }^{3}$ Warren Alpert Medical School of Brown University, Providence, RI, ${ }^{4}$ Department of Orthopaedics, Division of Spine Surgery-Adult Spinal Deformity Service, Warren Alpert Medical School of Brown University, Providence, Rhode Island

Background/Introduction: Many risk factor analyses utilize the NSQIP and NIS datasets, but such investigations are seldom directly compared. Data in NSQIP and NIS are collected differently, and therefore each possesses unique strengths and weaknesses. Precisely because of these differences, comparative analysis of the NSQIP and NIS may yield results that offer increased validity over single database studies. This investigation analyzed tobacco use and its association with complications following spinal fusion. 
Materials/Methods: In total, 56,145 NSQIP patients and 1,311,426 weighted NIS patients undergoing spinal fusion were analyzed. The primary outcome measures were perioperative complications. NSQIP complications were stratified based on occurrence before vs. after discharge. Descriptive statistics and bivariate analyses were generated. Multiple logistic regression was employed in adjusted analyses; a binomial and multinomial approach was used for the NIS and NSQIP, respectively.

Results: Among NSQIP and NIS patients, 24.0\% and 31.8\% were identified as tobacco users, respectively. Prior to discharge, NSQIP smokers had increased odds of pneumonia (OR 1.43, $\mathrm{p}=0.0146)$, postoperative intubation (OR 1.40, $\mathrm{p}=0.0306)$, and sepsis $(\mathrm{OR} 1.71$, $\mathrm{p}=0.0136)$, and decreased odds of PE (OR 0.57, $\mathrm{p}=0.0383$ ). After discharge, NSQIP smokers exhibited increased odds of cardiac arrest (OR 2.53, $\mathrm{p}=0.0417)$ and SSI (OR 1.25, $\mathrm{p}=0.0087)$, and decreased odds of UTI (OR 0.68, $\mathrm{p}=0.0121)$ and DVT (OR 0.61, p=0.0212). In adjusted NIS analysis, tobacco users exhibited increased odds of inpatient pneumonia (OR 1.57, $\mathrm{p}<0.0001)$, MI (OR 1.29, $\mathrm{p}=0.0413)$, and postoperative intubation $(\mathrm{OR} 1.15$, $\mathrm{p}=0.0156)$, and decreased odds of PE (OR 0.80, $\mathrm{p}=0.0488$ ) and DVT (OR 0.78, $\mathrm{p}=0.0221)$.

Discussion/Conclusion: Spinal fusion patients with a history of tobacco use are at increased risk of postoperative pneumonia and intubation, but a decreased risk of DVT and PE during the inpatient postoperative stay. Following discharge, smokers exhibited increased odds of cardiac arrest and SSI. The results from the NIS and NSQIP datasets were very similar overall, with 10 of 12 outcomes exhibiting comparable conclusions. This investigation provides important data regarding the perioperative risks of smoking in spinal fusion patients, and demonstrates how a qualitative comparison of NIS and NSQIP datasets yields results that likely offer increased validity over single data source studies.

Poster 24. Impact of iliac crest bone grafting on postoperative outcomes and complication rates following minimally invasive transforaminal lumbar interbody fusion

\section{Brittany Haws, $B S^{l}$, Benjamin Khechen, $B A^{l}$, Kaitlyn Cardinal, $B S^{1}$, Jordan Guntin, $B S^{1}$, Chen Chen, $H S^{1}$, Kern Singh, $M D^{2}$ \\ ${ }^{1}$ Rush University Medical Center, Chicago, Illinois, ${ }^{2}$ Rush University Medical Center, Chicago, IL}

Background/Introduction: Iliac crest bone grafting (ICBG) is an effective graft option for lumbar fusion procedures. Previous studies have reported an association between ICBG and increased postoperative pain and morbidity. The relationship between bone graft technique and postoperative outcomes for minimally invasive transforaminal lumbar interbody fusion (MIS TLIF) has not been well defined. This study aims to determine the effect of ICBG on patient reported outcomes and complication rates following MIS TLIF.

Materials/Methods: Primary, single-level MIS TLIF patients were consecutively analyzed. A prospective cohort of patients that received a percutaneous technique of ICBG was compared to a retrospective cohort of patients that received Bone Morphogenic Protein-2 (BMP-2). A tubular dilator was utilized to harvest cancellous iliac crest graft $(40 \mathrm{cc})$. Baseline demographics and operative characteristics were compared between groups. Complication rates were assessed for the perioperative period and up to 6-months postoperatively. Postoperative changes in Oswestry Disability Index (ODI), Visual Analog Scale (VAS) back and VAS leg pain from preoperative values were compared between cohorts. Rates of minimum clinically important difference (MCID) achievement at 6-month follow-up for ODI, VAS back, and VAS leg scores was compared between groups.

Results: 92 patients were included, with 46 in each cohort. No significant differences in preoperative characteristics existed between cohorts. Significant increases in operative time $(13.33$ minutes, $\mathrm{p}=0.016)$ and estimated intraoperative blood loss $(15.54 \mathrm{~mL}, \mathrm{p}=0.030)$ were demonstrated for the ICBG cohort. A greater percentage of ICBG patients were discharged on postoperative day $0(19.6 \%$ vs $14.4 \%, \mathrm{p}=0.072)$ though this did not reach statistical significance. No significant differences in complication rates were identified. The ICBG cohort demonstrated greater improvements in VAS leg pain at 12-week follow-up (-4.6 vs -3.2, p=0.032) compared to the BMP-2 cohort. No other significant differences in patient reported outcomes or rates of MCID achievement were identified.

Discussion/Conclusion: Patients undergoing MIS TLIF with ICBG experienced clinically insignificant increases in operative time and estimated blood loss. Use of ICBG did not lead to increases in pain, complications, pseudarthrosis rates, or delays in discharge. These results suggest that the use of ICBG is a safe and effective option for patients undergoing MIS TLIF.

Poster 25. Assessment of clinical outcomes and efficacy of minimally invasive transforaminal lumbar interbody fusion (TLIF) in obese patients with debilitating back pain from spondylolisthesis

Mick Perez-Cruet, $M D^{I}$, Esam Elkhatib, $M D^{2}$, Elizabeth Abel, $B S^{2}$

\section{${ }^{1}$, Royal Oak, Michigan, ${ }^{2}$, Royal Oak, MI}

Background/Introduction: Obesity is a major health problem in patients suffering from debilitating back pain that contributes to post-operative complications. Minimally invasive treatment (TLIF) can provide benefits by proving smaller incisions, reduced tissue destruction, less post operative pain, early ambulation, and less hospital stay.

Materials/Methods: Eighty-five patients (55(65\%) female, 30(35\%) male, average age 65 years old) with BMI greater than $30 \mathrm{~kg} / \mathrm{m} 2 \mathrm{with}$ grade I or II spondylolisthesis operated by TLIF were retrospectively reviewed between November 2011 to April 2017. These patients were categorized into three obesity categories: BMI $30.1-34.9 \mathrm{~kg} / \mathrm{m} 2$ (Class I), $35.0-39.9 \mathrm{~kg} / \mathrm{m} 2$ (Class II), and = $40.0 \mathrm{~kg} / \mathrm{m} 2$ (Class III). Oswestry disability index, Pain analogue scale, operative time, estimated blood loss (EBL), post-operative complications and hospital stay (LOS) and fusion rates were analyzed.

Results: Average follow-up time was 2.2 years. Obesity categories included the following: 40 class 1 , 29 class 2 , and 16 class 3 having chronic back pain symptoms averaging 5 years range 1 to 30 years. All patients underwent single level TLIF at the L3-4(n=6), L4-5(n= 58), or L5-S1 ( $n=21$ ) level. Mean operative time was 199 minutes +/- 45 minutes, EBL was $144 \mathrm{ml}+/-55 \mathrm{ml}$ and mean LOS was 4.4 days $+/-2.1$ days. VAS decreased from 6.55 pre-op to 2.8 at 3 months postoperative and 3.4 at one year, ODI decreased from 44.7 pre-op to 27.7 at 3 months post-op and 30.5 at one 
year. Complications rate was $4.9 \%$, fusion rate was $>93 \%$ at 3 and 12 months follow-up based on dynamic plain radiographs. Re- operation rates was $1.1 \%$ for adjacent level pathology.

Discussion/Conclusion: Obese patients with debilitating back pain from lumbar spondylolisthesis can be safely and effectively managed with minimally invasive TLIF to reduce complications, improve fusion rates, and provide excellent clinical outcomes.

Poster 26. Predictors of adverse discharge disposition in adult spinal deformity and associated costs

Peter Passias, $M D^{1}$, Cole Bortz, BA ${ }^{1}$, RABIA QURESHI, BS ${ }^{2}$, Bassel Diebo, MD ${ }^{3}$, Justin Paul, MD, PhD ${ }^{l}$, Samantha Horn, BA ${ }^{l}$, Frank Segreto, $B S^{1}$, Shay Bess, $M D^{4}$, Michael Gerling, $M D^{5}$, Hamid Hassanzadeh, $M D^{6}$ $V A$

${ }^{1}$, New York, NY ${ }^{2}$, CHARLOTTESVILLE, VA,${ }^{3}$, Brooklyn, $\mathrm{NY},{ }^{4}$, Denver, Colorado, ${ }^{5}$, TriBeCa, NY, ${ }^{6}$ University of Virginia, Charlottesville,

Background/Introduction: With advances in the understanding of adult spinal deformity (ASD), more complex osteotomy and fusion techniques are being implemented with increasing frequency. Patients undergoing ASD corrections infrequently require extended acute care, longer inpatient stays, and discharge to supervised care. Given the necessity of value-based health care, identification of clinical indicators for adverse discharge disposition in ASD surgeries is paramount. This study aims to identify predictors of adverse discharge disposition after ASD surgeries and view corresponding differences in charges.

Materials/Methods: Retrospective analysis of patients on the National Surgical Quality Improvement Program (NSQIP) database and cost data from Medicare PearlDiver Database. Patients >18 years undergoing thoracolumbar ASD-corrective surgery with a primary diagnosis of scoliosis (ICD-9 code 737.x) were isolated. Predictors (demographic, clinical, complications) of not-home (NH; rehab or skilled nursing facility) discharge were analyzed using binary logistic regression controlling for levels fused, decompressions, osteotomies, and revisions. Average 30-day and 90-day costs of care were reported in home, rehab, and skilled nursing facility discharge groups in patients undergoing 8+ level thoracolumbar fusion.

Results: 1,978 patients undergoing lumbar ASD-corrective surgery were included (average age:59.3 years, sex:64\%F). Average length of stay was 6.58 days. Multivariate regression analysis showed age over 60 years (OR: 0.28 CI:0.22-0.34) and female sex ( $\mathrm{p}=0.003$ ) as independent predictors of adverse discharge status. Partially dependent pre-operational functional status increased likelihood of adverse discharge disposition (OR:0.57 CI:0.35-0.90). Despite controlling for all clinical variables except for the ones specific to each analysis, Smith-Petersen osteotomy (OR:0.51 CI:0.40-0.64), interbody device placement (OR:0.80 CI:0.64-0.98) and fixation to the iliac (OR:0.54 CI:0.41-0.70) both increased likelihood of adverse discharge. Complications most associated with adverse discharge were UTIs (OR:0.34 CI:0.21-0.57) and blood transfusions (OR:0.42 CI:0.34-0.52). Relative to home-discharge, 30-day costs of care were $+\$ 21,061$ more expensive in rehab discharges, but not different in skilled nursing facility discharges $(+\$ 5,791, p=0.177)$. 90-day costs of care were $\$ 23,815$ in rehab discharges $(p<0.001)$, but not different in skilled nursing facility discharges $(+\$ 6,091, \mathrm{p}=0.212)$.

Discussion/Conclusion: Discharge destination to rehabilitation has a significant impact on cost of thoracolumbar adult spinal deformity surgeries. Patient selection can predict patients at higher risk for discharges to rehab or skilled nursing facility.

\section{Poster 27. Sustained Changes in Age-Adjusted Full-Body Compensation Following Adult Spinal Deformity Corrective Surgery}

Peter Passias, $M D^{1}$, Samantha Horn, $B A^{1}$, Cyrus Jalai, $B A^{1}$, Gregory Poorman, $B A^{2}$, Cole Bortz, BA ${ }^{1}$, Frank Segreto, BS ${ }^{1}$, Bassel Diebo, $M D^{3}$, Thomas Errico, $M D^{4}$, Virginie Lafage, $P h D^{5}$, Michael Gerling, $M D^{6}$

\section{${ }^{1}$, New York, $\mathrm{NY},{ }^{2}$, bronxville, ny, ${ }^{3}$, Brooklyn, $\mathrm{NY},{ }^{4}$ Department of Orthopedic Surgery, NYU Langone Orthopedic Hospital, NYU Langone Health, New York, New York, ${ }^{5}$ Hospital for Special Surgery, New York, NY, ${ }^{6}$, TriBeCa, NY}

Background/Introduction: Full body stereoradiographs allow for the assessment of lower limb compensation and global alignment in adult spinal deformity(ASD) patients, though little work has been done looking at how these recovery mechanisms change between early and late followup. The aim of this study was to evaluate the changes in lower limb compensation and global alignment for ASD patients from the early to late postoperative period.

Materials/Methods: Single-center retrospective review. ASD patients(SVA $>5 \mathrm{~cm}, \mathrm{PI}-\mathrm{LL}>10^{\circ}$, or PT $\left.>20^{\circ}\right)$ with full body stereographic $\mathrm{x}-\mathrm{rays}$ at baseline, 3 month(3M), 1 year(1Y) follow-up. Correction groups created to compare actual alignment with age-adjusted ideal values using established formulas for SVA, PI-LL, PT and TPA. Patients who matched("Match") within a 10-year range threshold for age-adjusted targets were compared to unmatched cases("Under"/“Over").

Results: 124 ASD patients(59yrs, 69\%F) had an average of 8.2 levels fused and $9 \%$ had three-column osteotomy use. Mean baseline alignment parameters were: $65 \mathrm{~mm} \mathrm{SVA}, 25^{\circ} \mathrm{PT}, 17^{\circ} \mathrm{PI}-\mathrm{LL}$, and $26^{\circ} \mathrm{TPA}$. At $3 \mathrm{M}$, patients significantly improved in TPA, TK, KA, GSA, and PS(all p<0.001). Patients that remained Undercorrected according to age-adjusted SVA ideals displayed significantly increased $\mathrm{KA}\left(8.3^{\circ}\right)$, posterior pelvic shift $(64.5 \mathrm{~mm})$, and GSA $\left(8.8^{\circ}\right)$ compared to Overcorrected patients(all $\left.\mathrm{p}<0.05\right)$. Of patients that Matched age-adjusted spino-pelvic ideals at Early follow-up, the rates of patients that subsequently degenerated to Undercorrected at Late follow-up were: SVA=22.6\%; PT=35.5\%; PI-LL=34.6\%; $\mathrm{TPA}=20.0 \%$. All spino-pelvic groups that went from Match-to-Undercorrected recruited an increasingly posterior pelvic shift(all p<0.046) with the exception of SVA cases. ASD patients with BMI $\geq 30$, and all spino-pelvic parameters displaying most severe alignment(++) were at significantly increased odds of degenerating from Match to Under between Early and Late follow-up.

Discussion/Conclusion: When ASD patients were undercorrected for SVA, PT, or PI-LL, they recruited increasingly posterior PS and KA, reflected overall with a greater GSA, to maintain upright balance. At baseline, an increase BMI and moderate-severe spinal deformity significantly predicted those patients that degenerated from Match to Undercorrection between Early and Late follow-up. 
Poster 28. Preexisting lumbar spinal stenosis does not affect outcomes of lumbar microdiscectomy

Philip Louie, $M D^{1}$, Bryce Basques, $M D^{1}$, Michael Nolte, $M D^{2}$, Tarush Khurana, $B S^{3}$, Deven Carroll, MS ${ }^{3}$, Arya Varthi, MD ${ }^{4}, J u s t i n ~ P a u l, M D$, $P h D^{5}$, Edward Goldberg, $M D^{6}$, Howard An, $M D^{l}$

${ }^{1}$ Rush University Medical Center, Chicago, IL, ${ }^{2}$ Rush University Medical Center, Chicago, Illinois, ${ }^{3}$, Chicago, Illinois, ${ }^{4}$ Yale University , New Haven, CT, ${ }^{5}$, New York, NY, ${ }^{6}$, Chicago, IL

Background/Introduction: Despite how commonly lumbar spinal stenosis is encountered, it is unknown if preexisting degenerative or congenital lumbar spinal stenosis has implications in patients who develop lumbar herniated nucleus pulposus (HNP) requiring discectomy. The purpose of the present study was to compare lumbar microdiscectomy patients with and without concomitant lumbar spinal stenosis in terms of baseline characteristics and longer term clinical outcome measures.

Materials/Methods: We performed a retrospective cohort analysis of patients who underwent a primary lumbar. Patient reported outcomes were obtained in the form of Oswestry Disability Index (ODI) scores, Visual Analog Scales (VAS) scores for the back and leg, 12-Item Short Form Mental and Physical Survey (SF-12) scores, and the Veterans Rand 12-Item Health Mental and Physical Survey (VR-12) scores. Patients were also surveyed about their expectations and satisfactions following surgery.

Results: Overall, 134 consecutive patients who fulfilled our inclusion criteria were assessed. Average follow-up was $13.3 \pm 15.5$ months (range 3-61 months). There were 49 patients (36.6\% of all patients) with concomitant stenosis. Patients with concomitant stenosis were older (57.4 + 14.2 vs $42.5+14.1, \mathrm{p}<0.001)$ and had a greater number of operative levels $(\mathrm{p}<0.001)$ compared to patients with isolated HNP. While patients with stenosis had lower preoperative VAS back, VAS leg, and ODI scores compared to those without stenosis on bivariate analysis, these differences disappeared after baseline characteristics were controlled for through multivariate analysis. On multivariate analysis, there were no differences between preoperative and postoperative outcome measures. Additionally, no difference was found between patient groups for overall clinical improvement from preoperative to postoperative time points. Patients with stenosis had similar rates of reoperation and met or exceeded expectations compared to patients without stenosis

Discussion/Conclusion: Patients with concomitant lumbar stenosis who underwent lumbar microdiscectomy for HNP had similar clinical outcomes compared to patients with isolated HNP. Re-operation rates are similar between the 2 groups of patients. Both groups report being satisfied with their outcome with their expectations being met or exceeded.

Poster 29. 30-Day Emergency Department Visits After Primary Lumbar Fusion: Incidence, Reasons, Risk Factors, and Costs

Nikhil Jain, $M D^{1}$, John Brock, $B A^{2}$, Frank Phillips, $M D^{3}$, Safdar Khan, $M D^{4}$

${ }^{1}$ Ohio State University, Columbus, Ohio, ${ }^{2}$, Philadelphia, Pennsylvania,${ }^{3}$, Chicago, IL, ${ }^{4}$, Columbus, OH

Background/Introduction: Emergency department (ED) visits are associated with long wait times, high costs, low patient satisfaction, and are the source of almost half of all hospital readmissions. No study has previously analyzed 30-day ED visits after lumbar fusion from an etiological and cost perspective. Our objective was to describe the incidence, reasons, risk factors, and costs associated with 30-day ED visits after primary lumbar fusion (PLF) for degenerative pathology in a large national patient cohort.

Materials/Methods: A national insurance database was used to study patients with PLFs performed for degenerative pathology of the spine between 2007 and Q3-2015. The incidence of all-cause, cause-specific 30-day ED visits, and ED to inpatient transfer were recorded. Risk factors for ED visits, and ED to hospital transfer were studied using multiple-variable logistic regression analysis. Total and average reimbursements for management of complications in ED, and hospital have been reported.

Results: Our cohort included 37,559 patients with a mean age of 66.0 10.0 years. From the overall cohort, 4,806 (12.8\%) patients had 10,281 ED visits within 30-days after surgery. Of all patients visiting the ED, $945(19.9 \%)$ had multiple ( $\geq 3)$ visits, and 1,466 (30.5\%) were admitted to the hospital for management. Common reasons for presentation in the ED were cardio-respiratory complaints (49.4\%), and back and/or leg pain (47.7\%). Several risk factors for all-cause ED visits, multiple ED visits, and hospital admission from the ED were identified. Additionally, patients visiting the ED once were far more likely to get transferred to inpatient care than patients with two or more ED visits (OR 44.1, 95\%CI:38.8-50.2). Based on our analysis, we propose two subsets of patients utilizing acute-care within 30-days after lumbar fusion (Fig 1). The overall ED cost burden was nearly two-thirds as much as hospital readmissions within 30-days $(\$ 6,994,260$ vs. $\$ 10,880,999)$.

Discussion/Conclusion: A sizable subset of patients present to the ED for acute care but do not require hospitalization. The reasons and risk factors for presentation in these patients are somewhat different from patients requiring readmission. Focus should also be directed on reducing frequency of ED visits after lumbar fusion to help improve quality and cost of care.

Poster 30. Preoperative PHQ-9 scores are not predictive of postoperative outcomes improvement after minimally invasive transforaminal lumbar interbody fusion

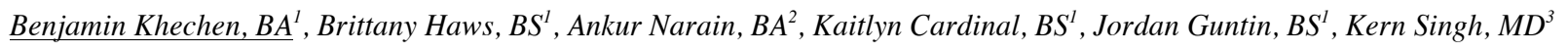

${ }^{1}$ Rush University Medical Center, Chicago, Illinois, ${ }^{2}$, Chicago, IL,${ }^{3}$ Rush University Medical Center, Chicago, IL 
Background/Introduction: Previous reports in the orthopedic literature have associated worse preoperative mental health with increased pain and reduced functional outcomes after joint arthroplasty. While similar reports exist within the spine literature, few have quantified patient mental health using validated mental health scoring scales such as the Patient Health Questionnaire-9 (PHQ-9). This study aims to utilize the PHQ-9 to determine if an association exists between preoperative mental health status and postoperative improvements in Oswestry Disability Index (ODI), Visual Analogue Scale (VAS) back pain, and VAS leg pain scores in patients undergoing single-level minimally invasive transforaminal lumbar interbody fusion (MIS TLIF).

Materials/Methods: A prospective surgical registry of patients undergoing primary, single-level MIS TLIF were retrospectively reviewed. Patient mental health was assessed preoperatively using the PHQ-9 survey, with higher scores corresponding to an increased severity of depression. Patient-reported outcome (PRO) measures including ODI, VAS back, and VAS leg pain scores were collected preoperatively and at 6-week, 12week, and 6-month postoperative time points. Multivariate linear regression was utilized to determine if an association existed between preoperative PHQ-9 score and preoperative ODI, VAS back, or VAS leg pain scores. Additionally, multivariate linear regression was used to test for an association between preoperative PHQ-9 score and postoperative improvements in PROs up to 6-months postoperatively

Results: 60 patients were included in this analysis. Worse preoperative mental health as evidenced by higher preoperative PHQ- 9 score was associated with increased preoperative ODI score (Coefficient: 0.94, 95\% Confidence Interval: 0.11-1.76, p=0.027). However, no association was demonstrated between preoperative PHQ-9 score and differences in PRO improvement for ODI, VAS back, or VAS leg pain scores at any postoperative time point ( $\mathrm{p}>0.05$ for each).

Discussion/Conclusion: Worse preoperative mental health was associated with higher perceived preoperative disability, but not preoperative pain, in patients undergoing MIS TLIF. Furthermore, worse preoperative mental health was not a predictor of impaired outcomes improvement after MIS TLIF. Surgeons should expect similar postoperative outcomes amongst their patients regardless of depressive symptom severity. Further work must be done examining psychosocial factors within spine surgery, and how they relate to postoperative outcomes after MIS surgery in particular with longer term follow-up.

Poster 31. Anterior versus posterior approaches for isthmic spondylolisthesis. A retrospective analysis

Emre Yilmaz, $M D^{1}$, Fernando Alonso, $M D^{2}$, Thomas $O^{\prime}$ Lynnger, $M D, M P H^{1}$, Tamir Tawfik, $M D^{l}$, Amir Abdul-Jabbar, MD ${ }^{l}$, Ronen Blecher, $M D^{1}$, Rod Oskouian, $M D^{3}$, Jens Chapman, $M D^{3}$, Christian Fisahn, $M D^{4}$

\section{${ }^{1}$ Swedish Neuroscience Institute, Seattle, WA, ${ }^{2}$ University Hospitals of Cleveland, Cleveland, OH, ${ }^{3}$, Seattle, WA, ${ }^{4}$ Swedish Neuroscience Institute, Swedish Medical Center, Seattle, Washington}

Background/Introduction: Surgical approaches for isthmic spondylolisthesis can be performed anteriorly, posteriorly or in combination. There are no strict guidelines on which method to use, and approaches are often selected based on surgeon preference. The purpose of this retrospective study is to compare patients who underwent posterior or anterior approaches for isthmic spondylolisthesis at a single institution and evaluate outcomes and assess complications.

Materials/Methods: Demographic information's, length of stay and opioid use was documented. Complications were assessed, including CSF leak, infection, suboptimal hardware placement, urinary tract infection, readmission, and reoperation. The pre- and post-operative measurements of spondylolisthesis and degree of lumbar lordosis were quantified using lateral x-rays. Post-operative improvement in radiculopathy was documented. Fusion rates at one and two year follow-up as assessed on lateral x-ray were documented. Univariate analysis was performed to compare demographics, surgical characteristics, complications, and pain outcomes. For categorical variables, frequency counts were computed and presented along with their percentages. For continuous variables, means were computed and presented along with their standard deviation. To compare categorical variables, the Chi-square test was used. For continuous outcomes, simple linear regression was used. Statistical significance was set at $\mathrm{p}<0.05$.

Results: Between 2010 and 2015, fifty-four patients underwent surgical treatment for isthmic spondylolisthesis. All procedures took place at either L5/S1 or L4/5. Fourteen patients underwent anterior lumbar interbody fusion, and forty patients underwent a posterior approach. There were no significant differences in patients' demographics, spondylolisthesis parameter, fusion rates, or complications. The posterior approach group showed a significantly decreased length of stay $(2.8 \pm 1.9$ days vs. $4.9 \pm 1.3$ days; $\mathrm{p}<0.01)$. The anterior approach group demonstrated improved lumbar lordosis compared to the posterior group $\left(62.8^{\circ} \pm 9.3 \mathrm{vs} .55 .3^{\circ} \pm 10.6 ; \mathrm{p}=0.03\right)$ as well as greater improvement in symptoms of radiculopathy $(92.9 \%$ vs. $85 \%$; $=0.03$ ).

Discussion/Conclusion: Anterior and posterior approaches to treating isthmic spondylolisthesis showed similar results overall. Posterior approaches had a decreased length of stay. Anterior approaches were associated with statistically significantly higher post-operative lordosis and a higher degree of improvement in symptoms of radiculopathy. However, results with both approaches were generally satisfactory and choice of approach will be dictated by anatomic considerations as well as surgeon preference and comfort.

Poster 32. Postoperative outcomes following primary and revision minimally invasive transforaminal lumbar interbody fusion

Benjamin Khechen, $B A^{1}$, Brittany Haws, $B S^{1}$, Ankur Narain, $B A^{2}$, Fady Hijji, $M D^{2}$, Benjamin Mayo, MD ${ }^{3}$, Kaitlyn Cardinal, BS ${ }^{1}$, Jordan Guntin, $B S^{1}$, Kern Singh, $M D^{3}$

\section{${ }^{1}$ Rush University Medical Center, Chicago, Illinois, ${ }^{2}$, Chicago, IL, ${ }^{3}$ Rush University Medical Center, Chicago, IL}

Background/Introduction: Minimally invasive transforaminal lumbar interbody fusion (MIS TLIF) and MIS lumbar decompression (LD) are effective surgical options for the treatment of lumbar degenerative disease. For LD patients with recurrent symptomatology, MIS TLIF has been 
reported as a safe and effective option. Few studies have assessed differences in outcomes between patients undergoing a revision MIS TLIF as compared to a primary MIS TLIF. As such, the purpose of this study was to compare improvements in patient reported outcome (PRO) measures between patients undergoing primary and revision MIS TLIF.

Materials/Methods: A prospectively maintained surgical database of patients undergoing a primary or revision one-level MIS TLIF for degenerative pathology during 2014-2016 was reviewed. Patients were excluded if there was incomplete PRO data (preoperative, 6-week, 12-week, or 6-month postoperative visit). Procedure type was tested for association with demographics, and preoperative PRO scores using student's t-test and chi squared analysis for continuous and categorical variables, respectively. Improvements in PROs from preoperative values were compared between primary and revision cohorts using student's t-tests. Chi-squared analysis was utilized to compare rates of MCID achievement. Statistical significant was set at $\mathrm{p}<0.05$.

Results: 162 patients were included in this analysis. 131 underwent primary MIS TLIF and 31 underwent revision MIS TLIF following primary LD. There were no differences in demographics and peri-operative outcomes between groups. Both groups experienced similar improvements in PRO scores at all postoperative time points ( $\mathrm{p}>0.05$ for each). The percent of patients achieving MCID did not differ between the primary TLIF and revision TLIF cohorts for ODI, VAS back, or VAS leg scores ( $\mathrm{p}>0.05$ for each).

Discussion/Conclusion: The results of the present study demonstrate patients undergoing primary and revision MIS TLIF recorded similar perioperative characteristics and experienced similar changes in PROs. This finding supports the assertion that undergoing a primary decompression followed by a revision MIS TLIF does not compromise PROs when compared to primary MIS TLIF. As such primary and revision MIS TLIF patients can be managed similarly and expect similar improvements in PROs. To further understanding of differences in outcomes following primary and revision MIS TLIF, prospective, long-term studies are necessary.

Poster 33. Identifying characteristics predicting hospital-acquired conditions in vertebral open reduction internal fixations: an analysis of 1055 cases

Deepak Kaji, BS ${ }^{1}$, Khushdeep Vig, B.A.Biochemistry ${ }^{2}$, Chierika Ukogu, BA ${ }^{3}$, Jun Kim, MD ${ }^{3}$, Samuel White, BA ${ }^{4}$, Samuel Cho, MD ${ }^{5}$

\section{${ }^{1}$, New York, New York, ${ }^{2}$ Mount Sinai Medical Center, Flora, MS,${ }^{3}$, New York, NY, ${ }^{4}$ University of Virginia, New York, New York, ${ }^{5}$, NY, NY}

Background/Introduction: Hospital-acquired conditions (HACs) can significantly increase healthcare costs as well as patient morbidity and mortality. There is a paucity of literature identifying risk factors for HACs in patients undergoing open reduction internal fixation (ORIF) for vertebral fractures or dislocations. The purpose of this study was to identify the risk factors that are predictive of HACs to help clinicians identify which patients may have a higher risk of HACs.

Materials/Methods: This was a retrospective analysis of prospectively collected data from ACS-NSQIP database between 2010-2014. Patients undergoing ORIF of vertebral fractures and/or dislocations were identified and different independent risk factors were analyzed for their correlation with postoperative HACs (venous thromboembolism, urinary tract infection, and surgical site infections). Vertebral ORIF procedures occurring in the cervical spine were compared to thoracic and lumbar procedures with respect to rates of HACs. Univariate analysis was used to assess patient baseline characteristics, comorbidities, and perioperative outcomes. Multivariable stepwise logistic regression models were employed, adjusting for patient demographic, preoperative and patient comorbidities, to identify the clinical risk factors associated with HACs.

Results: 1055 vertebral ORIF cases were identified from 2010-2014, and 7.87\% of these patients experienced a HAC. HACs occurred in 7.37\% of cervical ORIF procedures, $12.90 \%$ of thoracic procedures, and $3.49 \%$ of lumbar procedures $(\mathrm{P}<0.001)$. Multivariate regression analysis showed that cervical procedures were just as likely to suffer HACs as both thoracic and lumbar procedures, but thoracic procedures trended toward an increased risk (Thoracic vs. Cervical. $\mathrm{OR}=1.725, \mathrm{P}=0.075$ ). Other independent risk factors for increased HACs included diabetes, both insulin and non-insulin dependent $(\mathrm{OR}=2.293 ; \mathrm{P}=0.003)$, and dependent functional status $(\mathrm{OR}=2.001 ; \mathrm{P}=0.037)$.

Discussion/Conclusion: The current study identified the specific risk factors predictive of postoperative hospital-acquired conditions with high accuracy in patients undergoing ORIF for vertebral fractures or dislocations. Our results indicate that diabetics and patients with preoperative dependent functional status should receive enhanced care in order to prevent these complications.

\section{Poster 34. Age and gender confound patient-reported outcomes measures (PROMS) in spine patients with back and neck pain}

David S. Jevotovsky, $B A^{l}$, Jared Tishelman, $B A^{l}$, Raj Karia, $M S^{l}$, Charla Fischer, $M D^{2}$, Aaron Buckland, $M D^{3}$, Thomas Errico, $M D^{l}$, Themistocles Protopsaltis, $M D^{3}$

${ }^{1}$ Department of Orthopedic Surgery, NYU Langone Orthopedic Hospital, NYU Langone Health, New York, New York, ${ }^{2}$ Columbia University Department of Orthopaedic Surgery, New York City, New York, ${ }^{3}$ Department of Orthopedic Surgery, NYU Langone Orthopedic Hospital, NYU Langone Health, Manhattan, NY

Background/Introduction: The Patient-Reported Outcomes Measurement Information System (PROMIS) has become increasingly popular due to their computer adaptive testing (CAT) methodology. The aim of this study is to explore how confounding variables such as age and gender affect PROMIS as compared to traditional PROMs in spine patients.

Materials/Methods: A retrospective analysis was performed on a database of PROMs at a single-institution. Patients who presented with a primary complaint of back or neck pain from 12/2016 to 4/2017 were included. The ODI, NDI, VASBack, VASNeck, VASArm, VASLeg, PROMIS Physical Function, PROMIS Pain Intensity, and PROMIS Pain Interference questionnaires were administered. The patient cohort was grouped based on complaint, age category $(18-44,45-64,65+)$ and gender (male or female). Age groups were matched to control for pain and gender. Gender was 
matched to control for age and VAS. To compare differences between gender, t-tests were performed. ANOVA was used for multivariate comparisons.

Results: 484patients with back pain and 128patients with neck pain were identified. Back and neck pain patients were matched into gender cohorts ( $\mathrm{N}=201$ in each group and 46 in each group, respectively). Among back pain patients, female patients demonstrated worse disability in terms of ODI (44.15vs38.45, p=0.005), whereas PROMIS Physical Functioning did not reveal differences by gender. Among neck pain patients, no significant differences were found between genders in traditional HRQLs or PROMIS when controlling for neck pain and age. Back pain and neck pain patients were matched into age cohorts ( $\mathrm{N}=135$ each and $\mathrm{N}=14$ each, respectively). Among back pain patients, ANOVA revealed differences between groups when controlling for back pain and gender: ODI $(\mathrm{p}<0.001)$, PROMIS Physical Function ( $\mathrm{p}=0.018)$ PROMIS Pain Intensity $(\mathrm{p}<0.001)$ PROMIS Pain Interference $(\mathrm{P}<0.001)$. Among neck pain patients, comparing matched age groups revealed significant differences in NDI ( $\mathrm{p}=0.032)$ and PROMIS Physical Function ( $\mathrm{p}=0.022)$, but not PROMIS Pain Intensity or PROMIS Pain Interference ( $\mathrm{p}>0.05)$.

Discussion/Conclusion: Age and gender are confounders of traditional HRQLs, as well as PROMIS domains. However the PROMIS CAT does offer age and gender specific scores which traditional PROMs lack. By accounting for confounding variables, PROMIS may be considered a superior research measure for spine patients.

\section{Poster 35. Phytochemicals combat the detrimental consequences of cigarette smoke on osteogenic differentiation}

Richard Pahapill, $B S^{1}$, Kevin Chang, $B S^{l}$, Chawon Yun, PhD ${ }^{2}$, Jonghwa Yun, N/A ${ }^{3}$, Soyeon Jeong, MS ${ }^{4}$, Ryan Lubbe, BS ${ }^{1}$, Adam Driscoll, BS ${ }^{1}$, Meraaj Haleem, $B S^{1}$, Wellington Hsu, M.D. ${ }^{5}$, Erin Hsu, PhD ${ }^{6}$

${ }^{1}$ Northwestern University Department of Orthopaedic Surgery, Chicago, Illinois, ${ }^{2}$ Northwestern University Department of Orthopaedic Surgery ,Chicago, IL ${ }^{3}$, Chicago, IL,${ }^{4}$ Northwestern University Department of Orthopaedic Surgery , Chicago, Illinois, ${ }^{5}$ Northwestern Memorial Hospital, Chicago, Illinois, ${ }^{6}$ Northwestern University Department of Orthopaedic Surgery, Chicago, IL

Background/Introduction: Cigarette smoking has been shown to inhibit bone healing and increase the risk of pseudarthrosis after spinal fusion. We previously reported that Aryl Hydrocarbon Receptor (Ahr) activation by dioxin, a prototype activator of the Ahr, inhibits bone regeneration and spine fusion in rats. We have also shown that dioxin-mediated inhibition of osteogenic differentiation is mitigated by co-treatment with Ahr antagonists in vitro. Since dozens of dioxin-like Ahr ligands are present in cigarette smoke, we now investigate the downstream mechanisms of cigarette smoke on osteogenic differentiation to identify potential therapeutic options to mitigate the effects on bone.

Materials/Methods: Isolated rat bone marrow stromal cells (BMSC) were cultured under standard or osteogenic conditions. BMSC were subsequently exposed to the vehicle control (DMSO) or Particulate Phase Extract (PPE), which was prepared from whole cigarette smoke. Some cells received co-treatment with Ahr antagonists, including: $\alpha$-naphthoflavone (ANF, synthetic antagonist); resveratrol (Res, stilbenoid found in grapes/red wine), and 3,3'-diindolylmethane (DIM, breakdown product of indole 3 carbinol present in vegetables). Known downstream markers of Ahr activation and osteogenic differentiation were quantified.

Results: PPE increased the activity of CYP1 family proteins (EROD assay), demonstrating clear Ahr activation. PPE also inhibited cell proliferation, ALP activity, and bone matrix mineralization. These inhibitory effects were at least partially mitigated by co-treatment with each of the Ahr antagonists, showing recovery of cell proliferation, ALP activity, and matrix mineralization. RNA and protein expression studies showed that PPE down-regulates numerous pro-osteogenic genes, such as ALP, RunX2, OCN and PHEX, whereas co-treatment with Ahr antagonists prevented PPE-mediated inhibition of those RNAs and proteins.

Discussion/Conclusion: Cigarette smokers are a historically difficult patient population for spine surgeons to treat due to increased rates of pseudarthrosis and complications following spinal fusion procedures. Our results suggest that Ahr hyper-activation may play an important role in the adverse effects of cigarette smoke on bone healing, and that Ahr antagonists are potentially protective in combating cigarette smoke-mediated inhibition of bone regeneration and healing. The next step is to explore the effects of cigarette smoke extract on bone regeneration and healing in vivo using a pre-clinical model of spine fusion.

Poster 36. Does kyphotic configuration on upright lateral radiograph correlate with radiographic instability in patients with degenerative lumbar spondylolisthesis?

Xu Sun, M.D. ${ }^{1}$, Xi Chen, $M D^{2}$, Zezhang Zhu, M.D. ${ }^{3}$, Yong Qiu, M.D. ${ }^{2}$

${ }^{l}$ Spine Surgery, Drum Tower Hospital, Nanjing University Medical School,, Nanjing, Jiangsu, ${ }^{2}$ Spine Surgery, Drum Tower Hospital, Nanjing University Medical School, Nanjing, Jiangsu, ${ }^{3}$, Nanjing, Jiangsu

Background/Introduction: Kyphotic configuration detected on neutral radiograph constitutes a distinct subgroup of degenerative lumbar spondylolisthesis (DLS). However, there is a paucity of data on the stability of DLS with kyphotic configuration, as well as what modalities can provide more accurate assessment of segmental stability at involved segment. This study was performed to investigate the the stability of DLS with a kyphotic configuration at the involved segment, and to determine the most useful diagnostic modalities in the evaluation of instability for such a situation.

Materials/Methods: This study reviewed the charts and radiographs of a consecutive series of patients with L4/5 DLS. The enrolled patients were divided into two groups based on slip configurations on the preoperative neutral radiographs: the kyphotic group (Group K) and non-kyphotic group (Group NK). The translational and angular motion was determined by comparing upright lateral radiograph (U) with a supine sagittal MR image (S) (combined, US) or flexion/extension radiographs (FE). Comparisons were made between the two groups with regards to translational and angular motion observed using US and FE. 
Results: There were 26 and 201 patients in Groups K and NK, respectively. In comparison to Group NK, Group K demonstrated significantly higher translational motion $(12.4 \%$ vs. $7.0 \%, \mathrm{P}<0.001)$ on US analysis, but significantly lower translational motion $(4.2 \%$ vs. $6.4 \%, \mathrm{P}<0.001)$ on $\mathrm{FE}$ analysis. Angular motion was detected to be significantly lower in US versus FE in Group NK $\left(1.2^{\circ}\right.$ vs. $\left.7.8^{\circ}, \mathrm{P}<0.001\right)$, while no significant difference in Group K ( $4.2^{\circ}$ vs. $\left.5.3^{\circ}, \mathrm{P}=0.132\right)$. In Group K, "instability" was recognized in $84.6 \%$ of patients using US versus $11.5 \%$ patients using FE $(\mathrm{P}<0.001)$; While in Group NK, no significant difference was observed in the incidence of "instability" between FE and US (31.3\% vs. $27.8 \%$, $\mathrm{P}=0.444)$. Overall, Group K had a significantly higher incidence of "instability" than Group NK (84.6\% vs. $31.3 \%, \mathrm{P}<0.001)$.

Discussion/Conclusion: DLS with a kyphotic configuration is a distinct subgroup highly frequently associated with the presence of segmental instability. The modality of US is shown to be superior to the traditional FE in measuring the translational motion and identifying "instability" for DLS patients with a kyphotic configuration.

Poster 37. The effect of preoperative symptom duration on postoperative outcomes following transforaminal lumbar interbody fusion Benjamin Khechen, $B A^{l}$, Brittany Haws, $B S^{l}$, Jordan Guntin, $B S^{l}$, Kaitlyn Cardinal, $B S^{l}$, Kern Singh, $M D^{2}$

\section{${ }^{1}$ Rush University Medical Center, Chicago, Illinois, ${ }^{2}$ Rush University Medical Center, Chicago, IL}

Background/Introduction: There exists a paucity of knowledge regarding the influence of preoperative symptom duration on patient reported outcomes (PROs) following lumbar fusion. In this context, this study aims to examine whether the time spanning from symptom onset to surgical intervention has an effect on postoperative clinical improvement in patients undergoing MIS TLIF.

Materials/Methods: Patients who underwent a primary, single-level MIS TLIF procedure for degenerative pathology from 2013-2016 were identified. Patients were grouped based on preoperative symptom duration ( $\leq 6$ months, $>6$ months). Pain was measured using the Visual Analogue Scale (VAS), and narcotic consumption was measured using Oral Morphine Equivalents (OMEs). Demographics and perioperative outcomes were compared using chi-squared analyses for categorical data and independent Student t-tests for continuous variables. PROs including Oswestry Disability Index (ODI), VAS back and VAS leg scores were collected at preoperative and postoperative visits (6-weeks, 12-weeks and 6 months). The percentage of patients achieving minimal clinically important difference (MCID) following surgery was compared between groups.

Results: 195 patients were included in this analysis. $80 \%$ had a symptom duration $>6$ months and the remaining $20 \%$ had a symptom duration $\leq$ 6 months. No differences in demographic or operative characteristics, inpatient pain, narcotic consumption and length of stay were identified between groups ( $>0.05$ for each). Patients with shorter symptom duration had significantly higher ODI scores preoperatively and had greater improvements in ODI scores at 6 weeks, 12 weeks, and 6 months ( $<<0.05$ for each). Shorter symptom duration patients also reported lower VAS Back scores at 6 months $(\mathrm{p}=0.044)$, but was not identified at 6 week or 12 week timepoints. VAS leg improvements were similar between groups at all postoperative time point. Additionally, a greater percentage of shorter symptom duration patients achieved MCID in ODI ( $\mathrm{p}=0.044$ ), however, this association was not demonstrated for VAS back or leg.

Discussion/Conclusion: Patients undergoing MIS TLIF with shorter symptom duration exhibited significantly higher preoperative ODI scores. However, these patients demonstrated greater clinical improvement postoperatively. These findings suggest delayed surgical intervention may lead to impaired functional recovery in patients with degenerative lumbar disease following MIS TLIF.

Poster 38. Posterolateral fusion (PLF) vs. transforaminal lumbar interbody fusion (TLIF) for low grade spondylolisthesis: a systematic review and meta-analysis

Jay Levin, $B S^{1}$, Joseph Tanenbaum, $B S^{2}$, Michael Steinmetz, $M D^{3}$, Thomas Mroz, M.D. ${ }^{4}$; Samuel Overley, $M D^{1}$

${ }^{1}$, Cleveland, $\mathrm{OH},{ }^{2}$ Case Western Reserve University School of Medicine, Cleveland, Ohio, ${ }^{3}$ Cleveland Clinic, Department of Neurosurgery, Cleveland, $\mathrm{OH},{ }^{4}$ Cleveland Clinic Foundation, Neurological Institute, Cleveland, $\mathrm{OH}$

Background/Introduction: Lumbar fusion is an effective and durable treatment for symptomatic lumbar spondylolisthesis, however the current literature provides insufficient evidence to recommend an optimal surgical fusion strategy. This study sought to compare the clinical outcomes, fusion rates, blood loss, and operative times between open posterolateral lumbar fusion (PLF) alone and open transforaminal lumbar interbody fusion + posterolateral fusion (TLIF) for spondylolisthesis.

Materials/Methods: An electronic database search was performed to identify investigations comparing PLF alone versus PLF + TLIF for treatment of low-grade lumbar spondylolisthesis. Effect size was assessed from pooling observational studies for each outcome variable, with odds ratios used for fusion and infection rate, mean difference used for improvement in ODI and leg pain as well as operative time and blood loss, and standardized mean difference used for improvement in back pain and health-related quality of life (HRQOL) outcomes. Studies were weighted based on the inverse of the variance and heterogeneity. Effect sizes from the meta-analysis were then compared to data from the RCTs.

Results: The initial literature search yielded 282 unique, English-language studies. Seven were determined to meet our inclusion criteria and were included in our qualitative analysis. Five observational studies were included in our quantitative meta-analysis. The pooled fusion success rates were $84.7 \%(100 / 118)$ in the PLF group and $94.3 \%(116 / 123)$ in the TLIF group (OR 0.33, $0.13-0.82, p=0.02)$. In regards to improvement in back pain, the effect size was $-0.27(-0.43$ to $-0.10, \mathrm{p}=0.002)$, in favor of the TLIF group. For ODI, the effect size was -3.73 (CI -7.09 to -0.38 , $\mathrm{p}=0.03)$, significantly in favor of the TLIF group. Operative times were significantly shorter in the PLF group, with an effect size of -25.55 (CI -43.64 to $7.45, \mathrm{p}<0.01)$. No significant difference was observed in leg pain, HRQOL improvement, blood loss, or infection rate. Our meta-analysis results were consistent with RCTs in favor of TLIF for achieving radiographic fusion and greater improvement in ODI and back pain

Discussion/Conclusion: Our results demonstrate that for patients undergoing fusion for spondylolisthesis, TLIF is superior to PLF with regards to achieving radiographic fusion and clinical improvement in disability and back pain. 
Poster 39. The effect of spinal fusion stimulators on outcomes following fusion procedures: a meta-analysis

Brittany Haws, $B S^{l}$, Benjamin Khechen, $B A^{l}$, Fady Hijji, $M D^{2}$, Ankur Narain, BA ${ }^{2}$, Daniel Bohl, MD, MPH ${ }^{3}$, Jordan Guntin, BS ${ }^{l}$, Kaitlyn Cardinal, $B S^{l}$, Kern Singh, $M D^{4}$

\author{
${ }^{1}$ Rush University Medical Center, Chicago, Illinois, ${ }^{2}$, Chicago, IL, ${ }^{3}$ Rush University Medical Center, New Haven, CT, ${ }^{4}$ Rush University \\ Medical Center, Chicago, IL
}

Background/Introduction: Despite the overall success of spinal fusions, pseudarthroses remain a common complication. Electrical stimulation devices have been popularized as an adjunctive tool to improve spinal fusion rates. There is minimal aggregate data from randomized controlled trials (RCT) to determine the overall efficacy of stimulators on spinal fusion outcomes. The objective of the current study was to perform a meta-analysis of RCTs assessing the effect of spinal fusion stimulators on postoperative fusion rates.

Materials/Methods: This meta-analysis was performed under the guidelines of the Preferred Reporting Items for Systematic Reviews and MetaAnalyses (PRISMA) guidelines. RCTs that compared fusion rates between cohorts receiving either electrical stimulation or a placebo/control following spinal fusion were included. Studies were obtained from PubMed and MEDLINE databases. Articles were excluded if they did not include a minimum follow-up period of 1 year or utilize complete randomization of patient cohorts. Fusion rate, study size, average age, number of smokers, type of electrical stimulation, spinal level, number of involved vertebral levels, type of fusion, graft type, instrumentation, and method of fusion assessment were recorded. A meta-analysis on relative risks for fusion was performed and the summary estimate was derived using Mantel-Haenszel methods.

Results: A total of 6 RCTs were included in this analysis. Fusion rates for the stimulation and control groups ranged from 35.4-90.6\%, and 33.3$92.8 \%$, respectively. Following pooling of fusion rates for each group, there was no significant difference in fusion rates between spinal stimulator and control groups. $(\mathrm{RR}=1.07 ; 95 \% \mathrm{CI}=1.00-1.15 ; \mathrm{p}=0.067)$. Additionally, 4 of 6 studies exhibited a high risk for bias.

Discussion/Conclusion: The results from the present study suggest no significant effect of spinal fusion stimulators on rates of spinal fusion when compared to control treatments. Physicians should use caution when interpreting these studies, as many are outdated and exhibit substantial variability. Further RCTs demonstrating uniformity in surgical indications, procedure, patient population, and stimulation device are necessary to better understand the effects of electrical stimulators on spinal fusion.

Poster 40. Underweight patients are the highest risk body mass index group for perioperative adverse events following anterior lumbar interbody fusion

Taylor Ottesen, B.S. ${ }^{1}$, Anoop Galivanche, B.S. ${ }^{2}$, Janelle Greene, $M D^{2}$, Rohil Malpani, B.S. ${ }^{3}$, Arya Varthi, MD ${ }^{4}$, Jonathan Grauer, MD ${ }^{5}$

${ }^{l}$ Yale University School of Medicine, New Haven, CT, ${ }^{2}$ Yale School of Medicine, New Haven, CT, ${ }^{3}$ Yale School of Medicine, New Haven, Connecticut, ${ }^{4}$ Yale University, New Haven, CT, ${ }^{5}$, New Haven, CT

Background/Introduction: Past studies investigating Body Mass Index (BMI) on spine surgery outcomes have focused on the effect of high BMI. These investigations have reported mixed conclusions, possible due to insufficient power, poor controlling of confounding variables, and inconsistent definitions of BMI categories (e.g. underweight, overweight, obese). Few studies have considered outcomes of patients with low BMI. The aim of the current study was to analyze whether anterior lumbar interbody fusion (ALIF) outcomes track with World Health Organization (WHO) categories of BMI to better assess where along the BMI spectrum patients become at risk for complications and mortality.

Materials/Methods: Patients undergoing ALIF surgery were abstracted from the 2005-2016 National Surgical Quality Improvement Program (NSQIP) database. Patients were excluded for a primary diagnosis of trauma, tumor, infection, or emergency presentation. Adverse and binomial outcome measures for 30-day post operation were abstracted from the database. Patients were then aggregated into WHO guidelines of BMI. Odds ratios of adverse outcomes, normalized to average risk of normal weight subjects (BMI 20-24.9), were calculated. Multivariate analysis was performed controlling for demographics (age, sex, functional status) and overall health as measured by the American Society of Anesthesiologists (ASA) classification.

Results: 17,319 ALIF patients met inclusion criteria. Odds for underweight patients were elevated beyond those observed in any other BMI category; however, both underweight and super obese class 3 patients were found to have significantly increased rates of adverse events and postoperative infection. There was no difference in these same variables between normal, overweight, obese class 1 or even obese class 2 patients. Moreover, overweight and obese patients showed a protective effect against mortality in both univariate and multivariate analysis.

Discussion/Conclusion: Both underweight and obese class 3 patients have higher rates of adverse events and post-operative infection than normal, overweight, obese class 1 or even obese class 2 patients as defined by WHO classifications. The current study identified underweight patients as an at-risk population that has previously not received significant focus. Physicians and healthcare systems should give additional consideration to this population, as they often already do for those at the other end of the BMI spectrum.

RF01. Prescriber education is effective in reducing opioid prescriptions at discharge after lumbar spine surgery

Francis Lovecchio, $M D^{l}$, Jeff Stepan, $M D, M S c^{l}$, Ajay Premkumar, MD, MSc ${ }^{2}$, Peter Derman, $M D^{l}$, Han Jo Kim, MD ${ }^{l}$, Todd Albert, $M D^{l}$

${ }^{l}$ Hospital for Special Surgery, New York, NY ${ }^{2}$ Hospital for Special Surgery, New York, ny 
Background/Introduction: Chronic back pain, a symptom in many patients undergoing lumbar spine surgery, is a known risk factor for developing an opioid use disorder. Provider education and prescribing guidelines may reduce overprescribing, decreasing the amount of pills available for diversion. The purpose of this study is to identify whether mandatory provider education and prescribing guidelines decreased the amount of opioids prescribed at discharge after lumbar spine surgery.

Materials/Methods: At an orthopedic specialty hospital, employees qualified to prescribe controlled substances were required to complete a mandatory one-hour program on responsible prescribing practices. An educational conference was given at various times over a two-month period (Nov-Dec 2016). Over the next two months (Jan-Feb 2017), consensus method among the spine surgeons was used to publish qualitative opioid prescribing guidelines. Electronic prescription data for 2479 patients who underwent lumbar spine surgery between March 2016 and October 2017 was captured, and patients were split into two groups depending on the timing of their surgery. Group 1 consisted of 1177 patients who underwent surgery in the period before prescriber education (Mar-Oct 2016, pre-education); Group 2, 1302 patients after prescriber education (Mar-Oct 2017, post-education). Surgeries were classified as decompression (i.e., no fusion or instrumentation) or fusion (instrumentation, including any form of interbody) procedures. Patients on long-acting opioids were excluded. Mann Whitney U and Pearson chi-square tests were used to compare continuous and categorical outcomes, respectively.

Results: For all lumbar spine surgeries, the mean amount of opioids prescribed at discharge was lower after the educational intervention and distribution of prescribing guidelines (629 \pm 294 OME pre-education vs. $490 \pm 245$ OME post-education, $\mathrm{p}<0.001)$. The mean number of tabs also decreased $(81 \pm 26$ vs. $66 \pm 22, \mathrm{p}<0.001)$. The greatest reduction in opioid prescription amount was seen in the decompression surgery category (Table 1). Analysis of the types of pills given revealed that weak opioids (tramadol) were prescribed more frequently after education (9.9\% vs. $18.6 \%$, $\mathrm{p}<0.001)$.

Discussion/Conclusion: In a population at high risk for opioid misuse, coupling provider education with qualitative prescribing guidelines is likely synergistic in decreasing the amount of opioids prescribed. The sustainability of these changes is yet to be determined.

RF02. Narcotic consumption following minimally invasive lumbar decompression: a comparison between hospital and ambulatory-based surgery centers

Benjamin Khechen, $B A^{1}$, Brittany Haws, $B S^{1}$, Dustin Massel, $M D^{2}$, Benjamin Mayo, $M D^{3}$, Kaitlyn Cardinal, BS $S^{1}, J o r d a n ~ G u n t i n, B S^{1}$, Kern Singh, $M D^{3}$

${ }^{1}$ Rush University Medical Center, Chicago, Illinois, ${ }^{2}$ University of Miami Hospital, Miami, FL, ${ }^{3}$ Rush University Medical Center, Chicago, IL

Background/Introduction: Several studies have compared outcomes between HBCs and ASCs following MIS LD. However, the association between narcotic consumption and pain in the immediate postoperative period has not been well characterized. Therefore, the objective of this study is to examine pain, narcotic consumption, and length of stay (LOS) among patients discharged on postoperative day (POD) 0 following a 1-level minimally invasive lumbar decompression (MIS LD) between hospital-based (HBC) or ambulatory surgery centers (ASC).

Materials/Methods: A surgical registry of patients who underwent a primary, 1-level MIS LD during 2013-2017 was reviewed. Patients were stratified by operative location. Differences between groups in patient demographics were assessed using independent sample t-tests for continuous variables and Chi-square analysis for categorical variables. The operative location and its effect on perioperative characteristics, inpatient pain scores and narcotics consumption was analyzed using multivariate linear regression adjusted for significant patient characteristics.

Results: A total of 235 patients were identified, of which $90(38.3 \%)$ and $145(61.7 \%)$ underwent surgery at a HBC or ASC, respectively. The HBC cohort exhibited an increased comorbidity burden $(\mathrm{p}=0.041)$ and had a greater percentage of privately insured patients $(\mathrm{p}=0.026)$. The HBC cohort recorded shorter operative time $(\mathrm{p}<0.001)$ and greater total estimated blood loss $(\mathrm{p}<0.001)$. Patients in the HBC cohort experienced prolonged LOS ( $\mathrm{p}<0.001)$, and consumed greater total OMEs (35.7 vs 15.0, $\mathrm{p}<0.001)$ compared to the ASC cohort. No differences were observed in the remaining outcomes.

Discussion/Conclusion: The results of the current study suggest that patients who underwent MIS LD at an ASC received fewer narcotics than patients treated at a HBC, which may contribute to shortened length of stay. Additionally, there was no difference in patient reported pain between cohorts despite the differences in narcotic utilization. As such, postoperative narcotics administration can be decreased without compromising pain control and may contribute to faster discharge.

RF03. PROMIS pain interference natural language: what does Your PROMIS pain score mean?

Jeremy Shaw, $M D^{1}$, Ross McEntarfer, $M D^{l}$, Natasha Greene, B.A. ${ }^{l}$, Angela Presson, PhD ${ }^{2}$, Chong Zhang, M.S. ${ }^{1}$, Brandon Lawrence, MD ${ }^{2}$, W. Ryan Spiker, $M D^{2}$, Nicholas Spina, $M D^{l}$, Darrel Brodke, $M D^{2}$

\section{${ }^{1}$ University of Utah, Salt Lake City, Utah, ${ }^{2}$, Salt Lake City, Utah}

Background/Introduction: Patient- reported outcome measures are an important tool for assessing health status across patient populations. Historically, commonly used outcome measures in spine care were narrow in scope and limited by the burden associated with their administration. The Patient-Reported Outcome Measurement Information System (PROMIS) was developed to overcome these limitations and provide a reliable and versatile testing system using item response theory and computerized adaptive testing. While PROMIS has been validated in the lumbar spine population, its use has been limited in part because many clinicians lack a common language understanding of the meaning and the significance of PROMIS scores. The purpose of this study was to develop plain language descriptions to apply to PROMIS Pain Interference (PI) scores.

Materials/Methods: We retrospectively analyzed prospectively collected PROMIS Bank v1.1 Pain Interference (PROMIS-PI) data for patients presenting for back and/or lower extremity complaints. Patients with missing scores, standard error $>0.32$, missing injury location, and assessments 
with $<4$ questions or more than 12 questions were excluded. PROMIS-PI scores from each encounter were grouped into buckets based on nonoverlapping confidence intervals. Common language descriptions based on published questions and answers were abstracted. Questions with fewer than 25 assessments were excluded from the bucket, as were those where fewer than $15 \%$ of assessments completed the question.

Results: In total, 14823 assessments and 6582 unique patients were included in the analysis. $94.3 \%$ of assessments were completed in 4 questions to generate the PROMIS-PI score. The number of assessments and unique patients were normally distributed. "Normal pain" was assigned a PROMIS-PI score of 50, with a higher number indicating increased pain interference. For the study cohort, the mean PI was $63.3 \pm 7.4$, median was 63 (IQR - 58,67) and the range was 0-84. Plain language descriptions to apply to PROMIS-PI scores are listed in Table 1.

Discussion/Conclusion: This study developed rational plain language descriptions to enhance both patient and physician understanding of the PROMIS Pain Interference domain. This data has broad utility in both the clinical and research settings. Additional work is required to evaluate patient and clinician perceptions of these novel natural language descriptions.

\title{
RF04. Predictive modeling for blood transfusion following adult spinal deformity surgery: a tree-based machine learning approach
}

\author{
Wesley Durand, $S c B^{1}$, J. Mason DePasse, $M^{2}$; Alan Daniels, $M D^{3}$
}

${ }^{l}$ Brown University, Warren Alpert Medical School, Providence, RI, ${ }^{2}$ Department of Orthopaedics, Warren Alpert Medical School of Brown University, Providence, Rhode Island, ${ }^{3}$ Department of Orthopaedics, Division of Spine Surgery-Adult Spinal Deformity Service, Warren Alpert Medical School of Brown University, Providence, Rhode Island

Background/Introduction: Blood transfusion is frequently necessary following or during adult spinal deformity surgery. Past models for transfusion risk among spine surgery patients are disadvantaged through use of single-institutional data, potentially limiting generalizability. We sought to develop predictive models for blood transfusion following adult spinal deformity surgery, utilizing both classification tree and random forest machine-learning approaches.

Materials/Methods: This investigation utilized the ACS NSQIP dataset years 2012-2015. Patients undergoing surgery for adult spinal deformity (ASD) were identified using primary-listed CPT codes. In total, 1,029 patients were analyzed. The primary outcome measure was intra-/postoperative blood transfusion. Patients were divided into training $(n=824)$ and validation $(n=205)$ datasets. Single classification tree and random forest models were developed. Both models were tested on the validation dataset using AUC, which was compared between models.

Results: Overall, $46.5 \%(\mathrm{n}=479)$ of patients received a transfusion intraoperatively or within $72 \mathrm{~h}$ postoperatively. The final classification tree model utilized operative duration, hematocrit, and weight, exhibiting AUC=0.79 (95\%CI 0.73-0.85) on the validation set. The most influential variables in the random forest model were operative duration, surgical invasiveness, hematocrit, weight, and age. The random forest model exhibited AUC $=0.85$ (95\% CI 0.80-0.90), indicating very good predictive capacity. Partial dependence plots of random forest variables uncovered non-linear relationships between preoperative variables and transfusion risk; these associations may be overlooked in past, regression-based models. The difference between the classification tree and random forest AUCs was non-significant $(\mathrm{p}=0.1551)$.

Discussion/Conclusion: This investigation produced tree-based machine-learning models of blood transfusion risk following ASD surgery. The random forest model offered very good predictive capability as measured by AUC. Our classification tree model offered superior ease of implementation as compared to the random forest approach, with a minor, non-significant decrease in AUC. Clinicians may choose to implement either of these models to predict blood transfusion among their patients. Furthermore, policy-makers may use this model on a population-based level to assess predicted transfusion rates following ASD surgery.

\section{RF05. Risk factors for perioperative complications in morbidly obese patients undergoing elective posterior lumbar fusion}

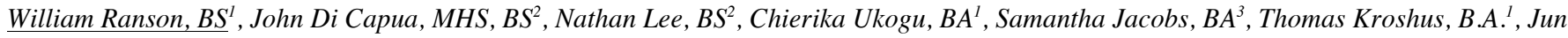
Kim, $M D^{I}$, Samuel Cho, $M D^{4}$

\section{${ }^{l}$, New York, NY, ${ }^{2}$ Icahn School of Medicine at Mount Sinai, New York, New York, ${ }^{3}$, New York, New York, ${ }^{4}, N Y, N Y$}

Background/Introduction: Obesity continues to be a growing problem in the United States, with a continuing rise in the prevalence of obesityrelated low back pain and degenerative disc disease. Past studies have demonstrated that obesity is associated with higher perioperative complication rates, but there remains a gap in the literature regarding additional risk factors that further predispose this already high-risk patient population to poor surgical outcomes following elective posterior lumbar fusion (PLF). This study seeks to utilize a national, multi-institutional database in order to identify independent risk factors for poor 30-day perioperative outcomes in morbidly obese patients undergoing PLF.

Materials/Methods: This is a retrospective cohort study of the American College of Surgeons National Surgical Quality Improvement Program (ACS-NSQIP) database from 2011 to 2014. We identified 22,909 patients in the ACS-NSQIP database who underwent elective PLF. There were 1,861 morbidly obese patients. Baseline patient demographics and medical comorbidities were collected. Univariate analysis was performed to compare perioperative complication rates between non-morbidly obese and morbidly obese patients. The five most common complications in the morbidly obese group were then selected for multivariate regression analysis to identify independent risk factors for poor 30-day outcomes.

Results: Morbidly obese patients had a higher perioperative complication rate. The five most common complications were prolonged hospitalization, blood transfusion, readmission, wound complications, and reoperation. Independent risk factors for these complications were age $\geq 65$ years, super obesity (i.e., BMI $>48.6$ ), chronic steroid use, ASA classification $\geq 3$, poor functional status, long length of fusion $\geq 4$ levels, and extended operative time (i.e., operative time $\geq 318$ minutes).

Discussion/Conclusion: Morbidly obese patients are at higher risk of perioperative complications following elective PLF. Modifiable risk factors for the most common complications are obesity and preoperative steroid use. 
${ }^{1}$ University of Tennessee, DiscGenics, Salt Lake City, UT, ${ }^{2}$ DiscGenics, Salt Lake City, UT, ${ }^{3}$ Semmes-Murphey Neurologic Institute, University of Tennessee, DiscGenics, Salt Lake City, UT

Background/Introduction: Cell therapy is a promising approach for treating lumbar degenerative disc disease. Our group has developed a method to generate allogeneic therapeutic progenitor cells for injection with a sodium hyaluronate carrier into painful degenerated discs. This rabbit study, which adheres to Good Laboratory Practices (GLP), was a critical part of the Investigational New Drug application that was recently allowed by the FDA.

Materials/Methods: The animal study evaluated the safety/toxicity and bioactivity of a low and high dose of IDCT compared to vehicle and sham in 48 male and female rabbits (under supervision of private IACUC). Two weeks after injury of L3-L4 and L4-L5, the discs were again surgically accessed and 251 of the treatment injected (or sham procedure performed). Throughout the study, clinical observations, ophthalmic examinations, body weights and qualitative food consumption were gathered. Disc height was evaluated via x-ray. Also, blood samples for hematology, coagulation, and clinical chemistry were collected. After 2 or 13 weeks, animals were euthanized. Macroscopic examination of the animals was performed, as well as numerous organs and tissues. The lumbar spines were excised from each animal, embedded in paraffin, sectioned and stained with hematoxylin and eosin (H\&E), Safranin-O, and Picrosirius red/Alcian blue. A blinded histological evaluation of the spine was performed with a novel scoring system.

Results: In this study, no treatment-related safety concerns were noted across any parameters. Animals administered Low or High dose showed significant improvement (increase in disc height) after dose delivery compared with sham animals or animals administered vehicle control article ( $\mathrm{p}<$ 0.05 , Figure A). Histologically, the low dose treatment at two weeks was more similar to healthy discs than the other groups (p $<0.05$, Figure B). No differences were noted by gender.

Discussion/Conclusion: In conclusion, no safety concerns associated with either dose of treatment or the vehicle were observed in the animals, both locally and systemically. The treatment had a regenerative effect as seen by a restoration of disc height and normalization of tissue architecture. Human clinical testing is under way.

\section{RF07. Effect of cannabinoid receptor activation on osteogenic differentiation}

Adam Driscoll, $B S^{l}$, Chawon Yun, PhD $D^{2}$, Soyeon Jeong, $M S^{3}$, Mark Oyer, MD ${ }^{1}$, Kevin Chang, BS $S^{1}$, Meraaj Haleem, BS ${ }^{l}, R y a n L u b b e, B S^{l}$, Richard Pahapill, BS ${ }^{1}$, Wellington Hsu, M.D. ${ }^{4}$, Erin Hsu, PhD ${ }^{5}$

${ }^{l}$ Northwestern University Department of Orthopaedic Surgery, Chicago, Illinois, ${ }^{2}$ Northwestern University Department of Orthopaedic Surgery, Chicago, IL, ${ }^{3}$ Northwestern University Department of Orthopaedic Surgery , Chicago, Illinois, ${ }^{4}$ Northwestern Memorial Hospital, Chicago, Illinois, ${ }^{5}$ Northwestern University Department of Orthopaedic Surgery, Chicago ,IL

Background/Introduction: Patients may experience significant pain following orthopaedic surgeries, which is currently treated with opioid medications. Given the potential for misuse and addiction with opioids, alternative post-operative analgesic strategies should be explored. The endocannabinoid system, a known regulator of neurotransmission and pain reception, has been shown to play a regulatory role in bone homeostasis and increase osteoblast numbers in vitro. Deficiencies of cannabinoid receptors CB1 and CB2 in osteoblasts and osteoclasts cause accelerated osteoporosis due to reduction of bone formation. Similarly, CB2 deficiency alone has been shown to result in enhanced bone turnover. WIN55.212-2 (WIN55) is an agonist of both CB1 and CB2 and may regulate post-operative pain. We sought to determine whether WIN55 has a quantifiable impact on osteogenic differentiation in primary bone marrow stromal cells (BMSC).

Materials/Methods: Rat BMSC, pre-sorted for positive expression of MSC markers, were purchased from Cell Biologics (Chicago, IL) and cultured under standard (SM) or osteogenic media (OM) conditions. Cells were subsequently exposed to either the vehicle control (DMSO) or various concentrations of WIN55. Alkaline phosphatase (ALP) activity, bone matrix mineralization, and expression levels of osteogenic differentiation marker genes (ALP and RUNX2) were quantified.

Results: Exposure to WIN55 did not inhibit ALP activity or bone matrix mineralization. mRNA expression of pro-osteogenic genes, such as ALP and RUNX2, was similar between DMSO and WIN55-treated cells, demonstrating that the CB1/2 agonist has no negative effect on the osteogenic differentiation capacity of rat BMSC.

Discussion/Conclusion: The opioid crisis has prompted strong interest in the development of alternative strategies for pain management after orthopaedic procedures. However, clear evidence that the treatment has no negative impact on healing would be required before consideration of any alternative pain management approach. We present proof-of-concept that WIN55, a cannabinoid receptor agonist, does not adversely affect osteogenic differentiation of primary rat BMSC in vitro. These results support the notion that endocannabinoids may present a valid alternative for post-operative analgesia following orthopaedic surgeries by reducing pain without inhibiting bone healing. We are currently exploring whether acute administration of WIN55 post-operatively impacts bone regeneration and healing capacity in vivo using a pre-clinical spine fusion model.

RF08. Factors influencing stability and treatment in grade 1 degenerative spondylolisthesis

Nicholas Spina, $M D^{l}$, Carlijn Schoutens, $B S^{1}$, Brook Martin, $P h D^{1}$, W. Ryan Spiker, $M D^{2}$, Brandon Lawrence, $M D^{2}$, Darrel Brodke, $M D^{2}$ 


\section{${ }^{1}$ University of Utah, Salt Lake City, Utah, ${ }^{2}$, Salt Lake City, Utah}

Background/Introduction: Several studies have recently questioned the optimal treatment for "stable" grade 1 lumbar spondylolisthesis. Yet diagnostic and treatment consensus amongst spine surgeons does not exist regarding what defines "stability". Classic parameters have been described based on cadaveric studies, but their translation into clinical practice remains imprecise and unclear. The purpose of this study was to understand how surgeons define "stability" and characterize surgical treatment patterns of stable and unstable grade 1 spondylolisthesis.

Materials/Methods: A survey was distributed to LSRS and AOspineNA members through REDCap. Members were queried as to the degree of static translation, dynamic translation, and angular change that would define an unstable lumbar motion segment. The relative importance of several other clinical and radiographic features were ascertained using a 5-point Likert Scale. Demographic data included subspecialty, primary practice setting, as well as preferred treatment method for both stable and unstable Grade 1 spondylolisthesis.

Results: A total of 226 respondents completed the survey. $75 \%$ were orthopedic, $25 \%$ neurosurgical trained. $59 \%$ surveyed practice in an academic setting with $41 \%$ of respondents practicing in a community, private practice, or VA setting. $99 \%$ of surgeons felt dynamic translation was moderately to extremely influential compared to slightly or not influential at all in defining stability whereas only $54 \%$ felt the same with regard to static translation. $68 \%$ of surgeons felt facet angulation was moderately to extremely influential, $67 \%$ for disc height, $47 \%$ for pelvic incidence, $51 \%$ severity of back pain, and $42 \%$ severity of neurogenic claudication. $60 \%$ of surgeons treated stable grade 1 spondylolisthesis with decompression alone vs $40 \%$ fusion. All but 2 respondents treated unstable spondylolisthesis with some type of fusion with $99 \%$ utilizing hardware. No clear consensus existed as to the type of decompression or fusion performed (Figure 1).

Discussion/Conclusion: A clear consensus regarding how surgeons define stability does not exist. Dynamic translation appears to be the most influential factor in that definition. Surgeons are divided on whether a fusion should be performed on "stable" grade 1 spondylolisthesis. Whereas a clear consensus exists that fusion is required when "instability" is present.

\section{RF09. Efficacy Of DBM compared To rhBMP-2 in posterior lumbar spine fusion}

Douglas Orndorff, MD, Jim Youssef, $M D$

\section{Spine Colorado, Durango, $\mathrm{CO}$}

Background/Introduction: For spine fusion, recombinant human bone morphogenetic protein-2 (rhBMP-2) is a popular and well-studied alternative to autograft. However, perceptions of higher performance and faster fusion rates have been challenged recently, and serious complications have been identified. These factors, combined with the high cost of rhBMP-2, has encouraged the use of alternative graft materials, such as demineralized bone matrices (DBMs). The primary objective of this study was to compare the efficacy of a DBM (Accell Evo3ß) to rhBMP-2 (Infuse ${ }^{\circledR}$ ) in posterior lumbar spine fusion.

Materials/Methods: Subjects requiring posterior lumbar spine fusion at one to three levels between L3 and S1 were enrolled in this IRBapproved study. Thirty subjects treated with DBM and local autograft were prospectively enrolled and compared to a retrospective cohort of 30 subjects treated with rhBMP-2 and local autograft. All patients received posterolateral fusion with pedicle screw instrumentation and the DBM or rhBMP-2 with local autograft. All DBM patients and all but six rhBMP-2 patients received transforaminal interbody fusion, with the DBM or rhBMP-2 used within the interbody cage at the discretion of the surgeon, along with local autograft within the disc space behind the cage. Subjects were followed for up to 24 months (range: 6-24 months). Fusion assessment was determined using X-ray or fine-cut CT, by a reviewer who was blinded to treatment. The Oswestry Disability Index (ODI) and the visual analog scale for back pain (VAS-back) were assessed.

Results: Fusion assessments were available for 29 DBM and 30 rhBMP-2 subjects. ODI and VAS-back data were available for fewer rhBMP-2 subjects than DBM subjects. The interbody and posterolateral fusion rates per level and per patient were similar in both groups, as were the improvements in ODI and VAS-back (Table). One rhBMP-2 subject experienced neuritis, possibly secondary to the use of rhBMP-2; no graft-related complications occurred in the DBM group. No subsequent operations were performed for pseudarthrosis in either treatment group.

Discussion/Conclusion: DBM combined with locally harvested bone had similar radiographic and clinical performance to rhBMP-2 in posterior lumbar spine fusion.

\section{RF10. Peptide amphiphile nanoscaffolds enhance the delivery of rh-BMP2 in a rabbit spine fusion model}

Meraaj Haleem, $B S^{1}$, Mark McClendon, PhD ${ }^{2}$, Ryan Lubbe, $B S^{1}$, Adam Driscoll, $B S^{1}$, Kevin Chang, BS ${ }^{1}$, Chawon Yun, PhD ${ }^{3}$, Soyeon Jeong, MS $S^{4}$ Richard Pahapill, BS $S^{1}$, Wellington Hsu, M.D. ${ }^{5}$, Erin Hsu, PhD ${ }^{6}$

${ }^{1}$ Northwestern University Department of Orthopaedic Surgery, Chicago, Illinois, ${ }^{2}$ Northwestern University Department of Materials Science and Engineering,, Chicago, IL, ${ }^{3}$ Northwestern University Department of Orthopaedic Surgery, Chicago, IL, ${ }^{4}$ Northwestern University Department of Orthopaedic Surgery, Chicago, Illinois, ${ }^{5}$ Northwestern Memorial Hospital, Chicago, Illinois, ${ }^{6}$ Northwestern University Department of Orthopaedic Surgery, Chicago, IL

Background/Introduction: Recombinant human bone morphogenetic protein (rhBMP-2) is an effective biologic to mitigate pseudoarthrosis after spinal fusion surgery. However, supraphysiologic doses of rhBMP-2 can lead to significant complications, necessitating development of a product that can reduce its therapeutic dose. Our previous work in a rat proof-of-concept model established peptide amphiphile (PA) nanofibers containing rhBMP-2 binding motifs as a potential material to achieve this aim. In this study, we utilized a rabbit posterolateral fusion (PLF) model to validate the efficacy of the BMP-2-binding PA nanofibers in a more stringent bone healing setting. 
Materials/Methods: Female New Zealand white rabbits underwent bilateral PLF at L4-L5 utilizing sub-therapeutic doses of $30 \mu \mathrm{g}$ or $60 \mu \mathrm{g}$ rhBMP-2 per animal (15 or $30 \mu \mathrm{g}$ per side). Rabbits received one of three delivery systems: ACS, PA/ACS, or PA/ACS particles. Radiography, manual palpation, and microCT imaging were utilized to establish bone regeneration and successful fusion. Manual palpation was performed by 3 blinded investigators using an established scoring system: $0=$ no fusion, $1=$ unilateral fusion, $2=$ bilateral fusion. Spines that average a score $\geq 1$ were considered fused.

Results: The delivery systems utilizing PA (PA/ACS or PA/collagen particles) achieved $100 \%$ fusion at both the $30 \mu \mathrm{g}$ or $60 \mu \mathrm{g}$ rhBMP-2 doses. This rate was significantly higher than the fusion rate observed utilizing ACS alone at either the $30 \mu \mathrm{g}(0 \%, \mathrm{p}<0.001)$ or $60 \mu \mathrm{g}(50 \%, \mathrm{p}<0.01)$ dose. Furthermore, both PA delivery formulations at either $30 \mu \mathrm{g}(2.00, \mathrm{p}<0.001)$ or $60 \mu \mathrm{g}(2.00, \mathrm{p}<0.01)$ had higher average fusion scores relative to ACS alone (1.04), suggesting a greater degree of bone formation.

Discussion/Conclusion: Although ACS is the only FDA approved rhBMP-2 delivery vehicle for spine fusion, its inefficient retention of the growth factor necessitates supraphysiologic doses to achieve consistent fusion. Our work demonstrates that a BMP-2-binding PA nanofiber scaffold used in conjunction with ACS or collagen particles can significantly potentiate rhBMP-2 action, thus reducing the necessary rhBMP-2 dose and potentially mitigating side effects associated with high doses of the growth factor. Future studies will establish the lower limit of the rhBMP-2 dose required to elicit fusion in the rabbit model.

RF11. Minimally Invasive versus Open Fusion for Grade I Degenerative Lumbar Spondylolisthesis: Analysis Of the Quality Outcomes Database $M . D{ }^{6}$

Praveen Mummaneni, $M D^{1}$; Panagiotis Kerezoudis, $M . D .^{2}$, Andrew Chan, $M D^{3}$, Christopher Shaffrey, $M D^{4}$, Paul Park, MD ${ }^{5}, M o h a m a d ~ B y d o n$,

${ }^{1}$, San Francisco, California, ${ }^{2}$ Mayo Clinic, Rochester, MN,${ }^{3}$ Department of Neurological Surgery, University of California, San Francisco, San Francisco, CA, ${ }^{4}$ University of Virginia Medical Center, Charlottesville, VA, ${ }^{5}$, Ann Arbor, Michigan, ${ }^{6}$, Rochester, MN

Background/Introduction: Lumbar spondylolisthesis is a degenerative condition that can be surgically treated with either open or minimally invasive decompression and instrumented fusion. Minimally invasive surgery (MIS) approaches may shorten recovery, reduce blood loss and minimize soft tissue damage with resultant reduced postoperative pain and disability.

Materials/Methods: We queried the national, multi-center, Quality Outcomes Database (QOD) registry from July 2014 through December 2015 for patients undergoing posterior lumbar fusion for grade I degenerative spondylolisthesis.

Results: A total of 345 patients (Open-254, MIS-91) from 11 participating sites were identified in the QOD. Follow-up rate at 12 months was $84 \%$. Overall, baseline patient demographics, comorbidities and clinical characteristics were similarly distributed between the two cohorts. Two hundred fifty seven patients underwent 1-level fusion (Open-181, MIS-76) and eighty eight patients underwent 2-level fusion (Open-73, MIS-15). Patients in both groups reported significant improvement in all primary outcomes (all $\mathrm{p}<.001)$. MIS was associated with significantly lower mean intraoperative blood loss and slightly longer operative times in both 1- and 2-level fusion subgroups. Although the length of stay was shorter for MIS 1-level cases, this was not significantly different. No difference was detected with regards to the 12-month patient reported outcomes between the one level mis versus the one level open surgical groups. However, change in functional outcome scores for patients undergoing 2-level fusion was notably larger in the MIS cohort for ODI (-27 vs. $-16, p=0.1)$, EQ5D (0.27 vs. $0.15, p=0.08)$, and NRS-BP (- 3.5 vs -2.7 , p=0.41); statistical significance was shown only though for NRS-LP scores $(-4.9$ vs. $-2.8, \mathrm{p}=0.02)$. On risk-adjusted analysis for one level fusion, open versus minimally invasive approach was not significant for 12-month PROs, length of stay and 90-day return to work.

Discussion/Conclusion: No difference was detected between the two techniques for 1-level fusion in terms of patient reported outcomes, length of stay and 90-day return to work. However, patients undergoing 2-level MIS fusion reported significantly better improvement in NRS-LP at 12 months. Longer follow-up is needed in order to further provide insight into the comparative effectiveness of the two procedures.

RF12. Obesity is associated with increased OR time, hospital stay, and postoperative wound complications in lumbar fusion surgery: analysis of 1,196 cases at a single institution

Amit Jain, MD, Sandra Hobson, MD, Eric Yoon, BS, Scott Boden, MD, John Heller, MD, John Rhee, MD, Tim Yoon, MD

\section{Emory University, Tucker, GA}

Background/Introduction: The aim of our study was to determine the association of obesity with operating room time, length of stay, hospital charges, and postoperative irrigation and debridement surgeries (I\&D) in patients treated with lumbar fusion surgery for degenerative pathologies.

Materials/Methods: A retrospective review of all lumbar surgeries for degenerative conditions was performed at a single orthopaedic hospital for a 4-year period from 2011 through 2015. Patients were stratified by their body mass index (BMI) into 4 categories: "Normal" (BMI 18.5-24.9), "Overweight" (BMI 25-29.9), "Obese" (BMI 30-34.9), and "Morbidly Obese" (BMI $\geq 35$ ). Multivariate linear regression analysis was performed and adjustments were made for patient age, comorbidities, levels fused, revision surgery status, and use of interbody device. Significance was set at $\mathrm{P}<0.05$ for all analyses.

Results: Of the 1,196 patients who underwent lumbar fusion surgery, 21.8\% were normal BMI (mean BMI: $22.7 \pm 1.6$ ), 39.4\% were overweight (mean: $27.5 \pm 1.5$ ), $26.2 \%$ were obese (mean: $32.1 \pm 1.4$ ), and $12.6 \%$ were morbidly obese (mean: $38.0 \pm 2.9$ ). A multivariate model revealed that compared to normal BMI patients, the adjusted mean operating room time was $25 \pm 6$ mins longer in the overweight group, $40 \pm 7$ mins longer in the obese group, and 50 \pm 8 mins longer in the morbidly obese group ( $\mathrm{P}<0.001$ each). Morbidly obese patients had significantly longer length of hospital stay compared to normal BMI patients ( 4.0 vs. 3.5 days, $\mathrm{P}=0.02$ ). Multivariate analysis revealed that the mean adjusted hospital charges for the index 
surgery were significantly greater in each of the higher BMI categories; compared to the normal BMI group, the total charges in the obese group were higher by $\$ 3,162 \pm \$ 1,325(\mathrm{P}=0.01)$, and in the morbidly obese group were higher by $\$ 4,738 \pm \$ 1,639$ ( $\mathrm{P}<0.01)$. The incidence of postoperative surgical I\&D increased significantly with BMI category $(\mathrm{P}=0.036)$; multivariate logistic regression analysis revealed that obese patients had 3.6-fold odds $(\mathrm{P}=0.040)$, and morbidly obese patients had 5.7-fold odds $(\mathrm{P}=0.012)$, of undergoing postoperative surgical I\&D compared to normal BMI patients.

Discussion/Conclusion: Higher BMI was associated with increased OR time, length of stay, total hospital charges, and postoperative surgical I\&Ds in patients treated with lumbar fusion surgery.

\section{RF13. Does vertebral endplate morphology impact on the disc height after transforaminal lumbar interbody fusion}

Xu Sun, M.D. ${ }^{1}$, Liang Xu, M.D. ${ }^{2}$, Yong Qiu, M.D. ${ }^{3}$, Zhen Liu, M.D. ${ }^{4}$, Zezhang Zhu, M.D. ${ }^{2}$

${ }^{l}$ Spine Surgery, Drum Tower Hospital, Nanjing University Medical School,, Nanjing, Jiangsu, ${ }^{2}$, Nanjing, Jiangsu, ${ }^{3}$ Spine Surgery, Drum Tower Hospital, Nanjing University Medical School, Nanjing, Jiangsu, ${ }^{4}$, Nanjing, Jiangse

Background/Introduction: Complication of cage subsidence and the resulting correction loss of disc height (DH) after transforaminal lumbar interbody fusion (TLIF) were found to significantly correlated with patient-reported outcomes. Previous studies reported that the degeneration of vertebral endplate was notably associated with the loss of DH before surgery. Whereas, its influence on (DH after TLIF has not been fully investigated. The aim of this study is to further analyze the sagittal morphology of the pair of endplates and its impact on postoperative DH.

Materials/Methods: A consecutive cohort of 125 patients with who underwent the TLIF due to lumbar disc herniation and associated canal stenosis at the level of L4-5 were reviewed. According to the morphology of the pair of L4-5 endplates on midsagittal MRI images, patients were divided into three groups: concave group $(n=31)$, parallel group $(n=59)$ and irregular group $(n=35)$. Comparisons were made with regards to DH, complications, and clinical outcomes among the three groups.

Results: The mean age was $52.1 \pm 14.5$ years and the minimum follow-up was 24 months. Before surgery, significant lower DH and higher VAS scores (including VAS leg pain and back pain) were observed in the irregular group when compared with the parallel group and the concave group $(\mathrm{P}<0.05)$. After surgery, considerable increase of $\mathrm{DH}$ was noted in the irregular group $(\mathrm{P}<0.05)$ while no significant difference was observed among three groups $(\mathrm{P}>0.05)$. During the follow-up, significant decrease in the $\mathrm{DH}$ in the irregular group was noted $(\mathrm{P}=0.021)$ and was notably lower than the other two groups $(\mathrm{P}<0.05)$. Besides, the correction loss of segmental lordosis, the incidence of cage subsidence, and the scores of VAS back pain in the irregular group was significantly higher in the irregular group $(\mathrm{P}<0.05)$.

Discussion/Conclusion: The correction loss of DH and the cage subsidence during follow-up in the irregular group was significant higher than that of the parallel group and the concave group. Additionally, considerable negative influence on clinical outcomes was also noted in the irregular group.

\section{RF14. Timing of adverse events following lumbar laminectomy with or without fusion}

Benjamin Howie, $B A^{1}$; Joseph Tanenbaum, BS ${ }^{2}$,Edward Benzel, M.D. ${ }^{3}$, Michael Steinmetz, MD ${ }^{4}$, Thomas Mroz, M.D. ${ }^{3}$

${ }^{1}$, Cleveland, Ohio, ${ }^{2}$ Case Western Reserve University School of Medicine, Cleveland, Ohio, ${ }^{3}$ Cleveland Clinic Foundation, Neurological Institute, Cleveland, $\mathrm{OH},{ }^{4}$ Cleveland Clinic, Department of Neurosurgery, Cleveland, $\mathrm{OH}$

Background/Introduction: Complications and risk factors associated with lumbar laminectomy, with and without fusion, have been extensively described in the literature. However, the timing of these adverse events is unknown. As efforts to discharge patients sooner post-operatively continue to strengthen, identifying time from operation to adverse event is essential in optimizing patient outcomes.

Materials/Methods: A prospective cohort analysis of the American College of Surgeons National Surgical Quality Improvement Program database for years 2012 through 2015 was performed. Patients undergoing lumbar laminectomy alone and laminectomy with fusion were analyzed. Analysis included incidence rate of adverse events, timing to adverse events, 30-day readmission rates and timing, and 30-day unplanned reoperation rates and timing.

Results: 36,664 patients were analyzed for the laminectomy group and 9,361 for the laminectomy with fusion group. For the laminectomy group, the median post-operative day until adverse events (with interquartile range) were as follows: $\bullet$ for stroke/CVA, 3 days (2-7 days), $\bullet$ myocardial infarction 3 days (2-5 days), pneumonia 4 days (3-8 days), $\bullet$ pulmonary embolism 7 days (2-17 days), $\bullet$ urinary tract infection 8 days (4-16 days), $\bullet$ deep vein thrombosis/thrombophlebitis 9 days (5-17 days), $\bullet$ unplanned reoperation 12 days (5-20 days), readmission 13 days (7-21 days). Similarly, for the laminectomy with fusion group, the median post-operative day until adverse event (lower quartile-upper quartile) were as follows: $\bullet$ stroke/CVA was 3 days (1.5-6 days), $\bullet$ myocardial infarction 4 days (2-5 days), $\bullet$ pneumonia 5 days (3-9.5 days), $\bullet$ pulmonary embolism 7 days $(2-14$ days), $\bullet$ urinary tract infection 8 days (4-16 days), $\bullet$ deep vein thrombosis/thrombophlebitis 11 days (5-19 days), $\bullet$ unplanned reoperation 13 days (620 days) $\bullet$ readmission 14 days (8-20 days),

Discussion/Conclusion: As health care systems continue to prioritize early post-operative discharge following lumbar laminectomy, it is increasingly important to understand the natural history and timing of adverse events. The data in this study came from a multi-institutional database that can effectively guide local and national guidelines and protocols for the post-operative management of lumbar laminectomy (with or without fusion).

RF15. Static versus expandable interbody devices in minimally invasive transforaminal lumbar interbody fusion: comparison of radiographic and functional outcomes 


\section{${ }^{1}$ Rush University Medical Center, Chicago, Illinois, ${ }^{2}$ Rush University Medical Center, Chicago, IL}

Background/Introduction: Advances in instrumentation, such as the development of expandable interbody devices, have been made in order to improve surgical outcomes and patient satisfaction. Previous studies have investigated surgical outcomes of different interbody devices. However, few have analyzed differences in radiographic and patient reported outcomes (PROs) between expandable and static interbody devices. The purpose of this study is to evaluate differences in radiographic parameters and PROs following single-level MIS TLIF between static and expandable interbody devices.

Materials/Methods: Patients undergoing primary, single-level MIS TLIF between 2014-2017 were retrospectively identified. Radiographic measurements including lumbar lordosis (LL), segmental lordosis (SL), disc height (DH) and foraminal height (FH) were performed on lateral radiographs before and after MIS TLIF with a static or expandable articulating interbody device. Patient characteristics were compared using chisquared analysis and student's t-test for categorical and continuous variables, respectively. Radiographic outcomes and PROs were compared using paired and unpaired student's t-test.

Results: A total of 60 patients were included in this analysis, of which, 30 patients received expandable interbody devices and 30 patients received static interbody devices. There were no differences in demographics or perioperative characteristics between cohorts. Both static and expandable device cohorts demonstrated significant increases in $\mathrm{DH}, \mathrm{FH}$, and LL ( $\mathrm{p}<0.05$ for each). Patients receiving an expandable device demonstrated a significant increase in SL $(\mathrm{p}=0.048)$. The expandable device cohort exhibited significantly greater improvement in DH $(+4.4 \pm 2.2$ $\mathrm{mm}$ versus $+2.2 \pm 1.4 \mathrm{~mm}, \mathrm{p}<0.001)$ at final follow-up. Both surgical cohorts experienced significant improvements in ODI, and VAS back and leg at 6-months postoperatively ( $\mathrm{p}<0.001$ for each). When comparing PROs between surgical cohorts, no differences in improvement were identified.

Discussion/Conclusion: Patients reported significant improvements in PROs following MIS TLIF in both expandable and static interbody device cohorts. However, no differences in improvement were demonstrated between surgical cohorts. Although use of expandable interbody device led to greater increases in DH, this may not correlate with superior patient outcomes. As such, patients undergoing MIS TLIF can expect similar improvements in PROs, whether receiving a static or expandable interbody device.

RF16. Clinical and radiographical outcomes of S2-alar-iliac screw instrumentation via a freehand technique for lumbosacropelvic fixation in adult patients: minimum follow-up of two years

Wataru Ishida, $M D^{1}$, Benjamin Elder, $M D, P h D^{1}$, Seba Ramhmdani, $M D^{2}$, Nicholas Theodore, MD ${ }^{3}$, Ziya Gokaslan, M.D. ${ }^{4}$, Jean-Paul Wolinsky, M.D. ${ }^{5}$, Ali Bydon, M.D. ${ }^{5}$, Timothy Witham, MD ${ }^{6}$, Daniel Sciubba, $M D^{5}$, Sheng-Fu Lo, MD ${ }^{6}$

${ }^{1}$ Johns Hopkins University School of Medicine, BALTIMORE, Maryland, ${ }^{2}$, Baltimore, MD, ${ }^{3}$ Johns Hopkins School of Medicine, Baltimore, Maryland, ${ }^{4}$ Brown University, Providence, Rhode Island, ${ }^{5}$ Johns Hopkins Hospital, Baltimore, Maryland, ${ }^{6}$ Johns Hopkins University School of Medicine, Baltimore, $M D$

Background/Introduction: With recent advancement in instrumentation devices such as larger screws for sacropelvic regions, robotic surgery, and intraoperative CT scan as well as novel techniques such as the S2-alar-iliac (S2AI) technique, fixation and fusion procedures in the lumbosacropelvic spine have become safer and resulted in better outcomes than two decades ago. In our institution, we preferentially perform S2AI screw instrumentation via a freehand technique. Here we report its safety profile alongside long-term clinical and radiographical outcomes.

Materials/Methods: Between October 2010, and December 2015, 232 sacropelvic fusion procedures with pelvic screw instrumentation were performed in 205 patients. The inclusion criteria were as follows: patients (1) implanted with S2AI screws and (2) with more than two-year follow-up periods, which yielded 72 patients with $150 \mathrm{~S} 2 \mathrm{AI}$ screws. The screw starting point was about $25 \mathrm{~mm}$ inferior to the superior aspect of S1 and $22 \mathrm{~mm}$ lateral to the midline on the coronal plane. S2AI screws were routinely directed toward $40-50^{\circ}$ lateral on the axial plane and $20-40^{\circ}$ caudal on the sagittal plane. Rates of complications such as pelvic screw breach, neurological deficits, screw loosening, reoperation as well as other operative and clinical outcomes were collected and statistically analyzed.

Results: Baseline characteristics were as follows: mean age $60.9 \pm 10.1$ years, female $63.9 \%$, mean BMI $30.6 \pm 7.0$, deformity patients $65.3 \%$, oncological patients $11.1 \%$, and osteoporotic patients $19.4 \%$. In terms of immediate postoperative complications, four S2AI screw breach (2.7\%) were noted in three patients without any damage to major vessels, organs, and nerves, which caused no sequelae and thus prompted no revision surgeries. With the mean follow-up periods of 33.3 \pm 8.3 months, long-term outcomes were acceptable with seven cases $(9.7 \%)$ of distal device breakage (L4-S2 rod fractures), all of whom underwent revision surgeries, three incidences (2.0\%) of major S2AI screw loosening ( $>2 \mathrm{~mm}$ radiolucency around the screws), seven incidences $(4.7 \%)$ of minor S2AI screw loosening $(<2 \mathrm{~mm})$, and six patients $(8.3 \%)$ with symptomatic L5-S1 pseudoarthrosis.

Discussion/Conclusion: The freehand S2AI technique resulted in acceptable immediate and long-term radiographical outcomes as well as clinical outcomes. Future comparative studies are warranted to further investigate its roles in this modern era of intraoperative navigation.

RF17. Adult spinal deformity: the influence of BMI on achieving postoperative age-adjusted-alignment goals

Peter Passias, $M D^{l}$, Samantha Horn, $B A^{l}$, Frank Segreto, $B S^{l}$, Gregory Poorman, $B A^{2}$, Dennis Vasquez-Montes, $M S^{l}$, Cole Bortz, $B A^{l}$, Shaleen Vira, $M D^{3}$, Aaron Buckland, $M D^{4}$, Michael Gerling, $M D^{5}$, Thomas Errico, $M D^{6}$ 


\begin{abstract}
${ }^{1}$, New York, NY,${ }^{2}$, bronxville, ny, ${ }^{3}$, New York, NY , ${ }^{4}$ Department of Orthopedic Surgery, NYU Langone Orthopedic Hospital, NYU Langone Health, Manhattan, NY, ${ }^{5}$, TriBeCa, NY, ${ }^{6}$ Department of Orthopedic Surgery, NYU Langone Orthopedic Hospital, NYU Langone Health, New York, New York
\end{abstract}

Background/Introduction: The impact of obesity on lower-limb-compensation and spinal-sagittal-alignment is not well understood, especially when considering patients' age-adjusted alignment targets. This study investigates the effect of BMI on age-adjusted alignment targets and lowerextremity-compensatory-mechanisms after adult spinal deformity (ASD) corrective surgery.

Materials/Methods: Retrospective review of a single-center database. Inclusion criteria: pts $\geq 18$ yrs, full-body stereographic $\mathrm{x}$-rays (baseline and $1 \mathrm{Y}$ ) and met ASD criteria (Sagittal Vertical Axis (SVA) $>5 \mathrm{~cm}$, Pelvic-incidence minus lumbar-lordosis (PI-LL) $>10^{\circ}$, coronal-curvature $>20^{\circ}$ or pelvic-tilt $\left.(\mathrm{PT})>20^{\circ}\right)$. Patients were stratified into age cohorts: $<40 \mathrm{y}, 40-65 \mathrm{y}, \geq 65 \mathrm{y}$ and obesity groups $(\mathrm{BMI}<25,25-30,>30)$. 1Y followup alignment was compared with age-adjusted ideal values for SVA, PI-LL, PT and T1 pelvic angle (TPA). Prevalence of patients who matched and unmatched (undercorrected or overcorrected) \pm 10 years age-adjusted targets was assessed. Health-related-quality-of-life (HRQL) scores, alignment and compensatory mechanisms were compared across cohorts using ANOVA and paired t-tests.

Results: 116 ASD patients $(62 \mathrm{yrs}, 66 \% \mathrm{~F})$ were included. Obese patients had less levels fused (6.73) compared to overweight (7.26) and normal (9.79) patients $(\mathrm{P}<0.05)$. Obese and overweight patients displayed worse SVA (49.83-obese, 52.43-overweight, 26.4-normal), TPA, PI-LL, and PT improvements postoperatively compared to normal-weight patients $(\mathrm{p}<0.05)$. Obese and overweight patients recruited more postoperative pelvic-shift (62.36-Obese, 49.80-Overweight, 31.50-Normal) and Global-Sagittal-Angle (GSA)(6.51-Obese, 6.35-Overweight, 3.40-Normal) compared to normal-weight patients $(\mathrm{P}<0.05)$. Obese and overweight patients were more under-corrected $(58.6 \%$-obese, $71.8 \%$-overweight, $37.8 \%$-normal weight) for PI-LL, $\mathrm{P}<0.05$. Obese patients of all ages displayed similar $(\mathrm{P}>0.05)$ lower-extremity compensation outcomes. Obese patients exhibited insignificant postoperative improvements to ODI, EQ5D, VAS Back and Leg, SRS component and total scores (P>0.05), and postoperative HRQL outcomes compared to overweight and normal patients $(\mathrm{P}<0.05)$.

Discussion/Conclusion: While all patients benefited from surgery, obese patients were under-corrected, had worse alignment and HRQL outcomes compared to normal patients. Obese patients also recruited more postoperative pelvic-shift and GSA. The benefits from age-adjusted alignment targets appear to be less substantial for obese patients.

RF18. Radiographic outcome and complications after single-level lumbar extended pedicle subtraction osteotomy for fixed global sagittal malalignment: a retrospective analysis of 55 adult spinal deformity patients with minimum 2-year follow-up

Thomas Buell, $M D^{1}$, Marcus Mazur, MD ${ }^{2}$, Jeffrey Mullin, $M D^{2}$, Juanita Garces, $M D^{2}$, James Nguyen, MD ${ }^{3}$, Davis Taylor, MD ${ }^{3}$, Chun-Po Yen, $M D^{2}$, Christopher Shaffrey, $M D^{4}$, Justin Smith, $M D^{5}$

${ }^{1}$ University of Virginia Health System, Charlottesville, Virginia,${ }^{2}$, Charlottesville, Virginia,${ }^{3}$ University of Virginia Health System , Charlottesville, Virginia , ${ }^{4}$ University of Virginia Medical Center, Charlottesville, VA, ${ }^{5}$ University of Virginia, Charlottesville, VA

Background/Introduction: In contrast to traditional pedicle subtraction osteotomy (PSO; Schwab grade 3 osteotomy), limited literature exists for extended PSO (ePSO; Schwab grade 4 osteotomy) in adult spinal deformity (ASD). Our study objective was to provide focused investigation of single-level lumbar ePSO for ASD patients with fixed sagittal malalignment (FSM).

Materials/Methods: Fifty-five consecutive ASD patients with FSM treated with single-level lumbar ePSO at our institution (from 2010 to 2015) were analyzed. Radiographic analyses included assessment of segmental lordosis through the ePSO (ePSO segmental lordosis; sagittal Cobb measured from the superior endplate of the vertebra above and inferior endplate of the vertebra below the ePSO), lumbar lordosis (LL), pelvic tilt (PT), pelvic incidence and LL mismatch, thoracic kyphosis (TK), and sagittal vertical axis (SVA) on standing scoliosis radiographs. Patients with 2year minimum follow-up were included and complications were analyzed.

Results: Mean postoperative improvement in ePSO segmental lordosis, overall LL, and SVA were $41^{\circ}(\mathrm{p}<0.001), 38^{\circ}(\mathrm{p}<0.001)$, and $12.5 \mathrm{~cm}$ $(\mathrm{p}<0.001)$, respectively. Early postoperative alignment was maintained at last follow-up (mean follow-up 52.3 months, range: $26.2-97.2$ months): ePSO segmental lordosis $\left(34^{\circ}\right.$ vs $\left.33^{\circ}, \mathrm{p}=0.270\right)$, LL (47.3 vs $\left.46.7^{\circ}, \mathrm{p}=0.339\right)$, and SVA $(4 \mathrm{~cm} \mathrm{vs} 5 \mathrm{~cm}, \mathrm{p}=0.330)$. Rod fracture $(\mathrm{RF})$ or pseudarthrosis (PA) adjacent to the ePSO occurred in 18.2\% (10/55) and 14.5\% (8/55), respectively. Accessory supplemental rods across the ePSO, a more recently employed technique, significantly reduced occurrence of $\mathrm{RF} / \mathrm{PA}$ on univariate $(\mathrm{p}=0.004)$ and multivariate analyses $(\mathrm{OR}=0.008,95 \%$ CI 0.0-0.606, p=0.029); this effect approached statistical significance on Kaplan-Meier analysis (log rank test, $\mathrm{p}=0.053$ ). Interbody cage placement at the ePSO produced greater ePSO segmental lordosis correction $\left(45^{\circ} \mathrm{vs} 35^{\circ}, \mathrm{p}=0.007\right)$ without significant increase in $\mathrm{RF} / \mathrm{PA}(\mathrm{p}=0.304)$. Transient and persistent motor deficits occurred in $14.5 \%(8 / 55)$ and $1.8 \%(1 / 55)$ of patients, respectively.

Discussion/Conclusion: ePSO is an effective technique to correct FSM for ASD. In comparison to traditional PSO, ePSO may allow greater focal correction with comparable complication rates, especially with interbody cage placement at the ePSO site and use of accessory supplemental rods. However, further prospective studies with direct comparison between traditional and ePSO are warranted prior to making definitive conclusions.

RF19. Massive increase in pediatric spinal fusions throughout the United States over a recent decade

Joseph Laratta, $M D^{1}$; Neil Kaushal, $M D^{2}$, Jamal Shillingford, $M D^{1}$, Zachary Ries, $M D^{3}$, Joseph Lombardi, $M D^{1}$, Comron Saifi, MD ${ }^{4}$, Hemant Reddy, $B S^{5}$, Ronald Lehman, $M D^{6}$, Lawrence Lenke, $M D^{2}$

${ }^{1}$ The Spine Hospital at Columbia University, New York, NY, ${ }^{2}$, New York, NY, ${ }^{3}$ Norton Leatherman Spine Center, Louisville, KY, ${ }^{4}$ Midwest Orthopedics at Rush, Chicago, IL, ${ }^{5}$ Northeast Ohio Medical University, Rootstown, $\mathrm{OH},{ }^{6}, \mathrm{New}$ York, $\mathrm{NY}$ 
Background/Introduction: Advancements in spinal instrumentation and correction techniques have given surgeons powerful new tools to perform long fusion constructs for pediatric scoliosis. With a focus on value-based care, it is essential to understand the overall performance, demographic and economic data surrounding pediatric spinal fusions involving 4 or more levels in the United States.

Materials/Methods: The Healthcare Cost and Utilization Project (HCUP) Kids' Inpatient Database (KID) was queried for patients who underwent fusion/refusion of 4-8 vertebrae (ICD-9-CM 81.63) or fusion/refusion of $\geq 9$ vertebrae (ICD-9-CM 81.64) between 2003 and 2012. The data represents weighted national estimates for children 0-17 years. Demographic and economic data were obtained which included the annual number of surgeries, age, sex, insurance type, location, and frequency of routine discharge.

Results: In 2012, the estimated total number of fusions involving 4-8 and $\geq 9$ vertebrae was 7,424 and 2,569 across the United States, respectively. The number of fusions involving 4 or more levels has increased $799 \%$ from 1,250 in 2003. Based on payer, private insurance comprised $59.3 \%$ of patients undergoing fusion of $\geq 4$ levels and Medicaid comprised another $32.6 \%$ of patients. Additionally, teaching facilities accounted for $94.0 \%$ of the procedures. In 2012 , girls were more likely than boys to undergo fusion ( $69.0 \%$ versus $31.1 \%$, respectively), and the procedure was more often performed in areas designated as not low-income ( $75.1 \%$ versus $22.8 \%$, respectively). The vast majority of procedures were performed in children's hospitals $(88.0 \%)$.

Discussion/Conclusion: Throughout the United States, there was a remarkable increase of $799 \%$ in the number of long fusion constructs in the pediatric population between 2003-2012. Further cost analyses are warranted to evaluate the overall societal impact of this marked increase.

RF20. Increased dislocation rates following total hip arthroplasty in patients with ankylosing spondylitis: an effect of abnormal spinopelvic motion?

Michael Stefl, $M D^{l}$, Jeffrey Wang, $M D^{2}$; Raymond Hah, $M D^{2}$, Nathanael Heckmann, $M D^{l}$, Hyanwoo Kang, MD ${ }^{l}$, Lawrence Dorr, $M D^{l}$

\section{${ }^{1}$, Los Angeles, CA, ${ }^{2}$ Keck School of Medicine of USC, Los Angeles, CA}

Background/Introduction: Patients with ankylosing spondylitis (AS) often experience chronic inflammation of the axial skeleton with resultant fusion and deformity of the spine. As such, patients with AS undergoing total hip arthroplasty (THA) may lack normal spinopelvic motion, predisposing them to impingement and dislocation. The purpose of this study was to evaluate dislocation rates following THA in patients with AS compared to patients lacking spine pathology, and evaluate the effect of age and gender as it relates to the risk of dislocation.

Materials/Methods: The PearlDiver database was utilized from 2005-2014 to identify patients who underwent a primary THA with and without a diagnosis of AS (ICD-9 720.0). Patients with AS were compared to a control group without AS. Patients in the control group were excluded if they had degenerative disk disease, an acquired spinal deformity, spondylosis, spondylolysis, spondylolisthesis, anomalies of the pelvic girdle including the SI joint, or a prior history of lumbar or thoracic spine surgery. Univariate and bivariate analyses were performed based on age and sex to evaluate dislocation rates at 90 days, 6 months, 1 year, 5 years, and 10 years post-operatively.

Results: A total of 4,540 patients (55.7\% male) were identified with AS who underwent a THA. These patients were compared to a control group of 829,104 patients $(40.0 \%$ male) without AS or known spine pathology. At final follow up, there were $17,922(2.16 \%)$ dislocations in the control group and 220 dislocations (4.85\%) in the AS group ( $<<0.0001)$. Patients with AS had a higher dislocation rate at all time points $(\mathrm{OR}$ range: 1.90 $2.31, \mathrm{p}<0.0001)$ compared to controls. Patients $>65$ years of age were over twice as likely to dislocate $(\mathrm{OR} 2.46, \mathrm{p}<0.0001)$ compared to age-matched controls, whereas there was no difference in dislocation rates between AS patients $<65$ years old and age-matched controls ( $p>0.05$ ).

Discussion/Conclusion: Patients with AS have higher rates of dislocation following THA than patients without AS. This effect is more pronounced amongst older patients with AS. This is likely related to decreased spinopelvic motion in the sagittal plane, predisposing patients to impinge and dislocate. Patients with AS are at increased risk of dislocation following THA.

RF21. Patients presenting with a lumbar far-lateral herniated nucleus pulposus can expect similar post-operative outcomes to those presenting with a central or paracentral herniation

Bryce Basques, $M D^{l}$, Philip Louie, $M D^{l}$; Michael Nolte, $M D^{2}$, Jeremy Mormol, $B S^{l}$, Deven Carroll, $M S^{3}$, Arya Varthi, MD ${ }^{4}, J u s t i n ~ P a u l, M D$, $P h D^{5}$, Edward Goldberg, $M D^{6}$, Howard An, $M D^{l}$

${ }^{1}$ Rush University Medical Center, Chicago, IL, ${ }^{2}$ Rush University Medical Center, Chicago, Illinois, ${ }^{3}$, Chicago, Illinois, ${ }^{4}$ Yale University , New Haven, CT, ${ }^{5}$, New York, NY, ${ }^{6}$, Chicago, IL

Background/Introduction: A far lateral herniated nucleus pulposus (HNP) consists of 7-12\% of all disc herniations. 'Far lateral' is often used to describe a lumbar disc herniation that compresses the exiting nerve root at the same level, lateral to the neural foramen. The goal of this study is to examine the pre- and post-operative outcomes between patients presenting with a central/paracentral versus a far lateral HNP.

Materials/Methods: We performed a retrospective cohort analysis of patients who underwent a primary lumbar microdiscectomy for lumbar spinal stenosis. Patients were divided into two groups based on the location of the HNP (central/paracentral versus far-lateral). Patient reported outcomes were obtained in the form of Oswestry Disability Index (ODI) scores, Visual Analog Scales (VAS) scores for the back and leg, 12-Item Short Form Mental and Physical Survey (SF-12) scores, and the Veterans Rand 12-Item Health Mental and Physical Survey (VR-12) scores.

Results: Overall, 212 consecutive patients fulfilled our inclusion criteria. Average follow-up was 24.14 months (range 6-78 months). There were 191 patients who presented with a central or paracentral HNP, while 21 patients presented with a far-lateral HNP. There were no significant differences in the demographic variables. Far lateral disc herniation patients presented with a trend towards a shorter duration of symptoms prior to surgery (24.5 vs 31.5 months; $p=0.052$. Far lateral disc herniation patients also presented with significantly lower SF-12 mental (52.14 \pm 13.49 vs 
$55.1 \pm 6.55 ; \mathrm{p}=0.034)$ and physical scores $(24.76 \pm 7.5$ vs $36.9 \pm 11.1 ; \mathrm{p}=0.008)$. Patients with far-lateral disc herniation also presented with significantly lower VR-12 mental $(47.2 \pm 12.8$ vs $56.9 \pm 7.0 ; \mathrm{p}=0.024)$ and physical scores $(25.8 \pm 8.7$ vs $39.38 \pm 11.8 ; \mathrm{p}=0.005)$. Post-operatively, all patients have similar SF-12 and VR-12 survey scores. The improvement in ODI score was significantly lower in the patients presenting with a far lateral disc herniation $(7.8 \pm 15.1$ vs $28.3 \pm 21.5 ; \mathrm{p}=0.002)$. No significant difference in re-operation rates was found.

Discussion/Conclusion: Despite compression at the site of the dorsal root ganglion and the reported greater difficulty in addressing far lateral disc herniations, patients with far lateral disc herniations can expect similar outcomes to those patients present with herniations in the central or paracentral region 2 years after surgery.

\title{
RF22. Misaligned incentives for lumbar spine surgery in the bundled payment for care initiative
}

Joseph Tanenbaum, $B S^{1}$, Edward Benzel, M.D. ${ }^{2}$, Michael Steinmetz, MD ${ }^{3}$, Thomas Mroz, M.D. ${ }^{2}$

${ }^{l}$ Case Western Reserve University School of Medicine, Cleveland, Ohio, ${ }^{2}$ Cleveland Clinic Foundation, Neurological Institute, Cleveland, $\mathrm{OH},{ }^{3}$ Cleveland Clinic, Department of Neurosurgery, Cleveland, $\mathrm{OH}$

Background/Introduction: Under the Bundled Payments for Care Initiative (BPCI), Medicare reimburses for lumbar fusion without adjusting for the patient's underlying pathology. We compared the hospital resource use of two lumbar fusion cohorts that BPCI groups into the same payment bundle: patients with spondylolisthesis and patients with thoracolumbar fracture.

Materials/Methods: With BPCI, hospitals are reimbursed for a lumbar fusion episode of care if patients are assigned diagnosis related group (DRG) 459 or 460 . Vertebroplasty and kyphoplasty are assigned different DRGs. National Inpatient Sample data from 2013 were queried to identify all patients that underwent lumbar fusion to treat a primary diagnosis of thoracolumbar fracture or spondylolisthesis and that were assigned DRG 459 or 460. Multivariable linear and logistic regression were used to compare length of hospital stay (LOS), direct hospital costs, and odds of discharge to a post-acute care facility for thoracolumbar fracture patients and spondylolisthesis patients. All models adjusted for patient demographics, 29 comorbidities, and hospital characteristics. The complex survey design of the NIS was taken into account in all models.

Results: After adjusting for patient demographics, insurance status, hospital characteristics, and 29 comorbidities, spondylolisthesis patients had a mean LOS that was 36\% shorter (95\% CI 26\% - 44\%, p<0.0001), a mean cost that was $13 \%$ less (95\% CI $3.7 \%-21 \%$, p<0.0001), and had 3.6 times greater odds of being discharged home $(95 \% \mathrm{CI} 2.5-5.4, \mathrm{p}<0.0001)$ than thoracolumbar fracture patients.

Discussion/Conclusion: Under the proposed DRG-based BPCI, hospitals would be reimbursed the same amount for lumbar fusion regardless of whether a patient had spondylolisthesis or thoracolumbar fracture. However, compared with fusion for spondylolisthesis, fusion for thoracolumbar fracture was associated with longer LOS, greater direct hospital costs, and increased likelihood of being discharged to a post-acute care facility. Our findings suggest that the BPCI episode of care for lumbar fusion dis-incentivizes treatment of trauma patients.

\section{RF23. Can the American College of Surgeons risk calculator predict 30-day complications after lumbar spine surgery?}

\author{
Michael McCarthy, $\mathrm{MD}^{l}$, Tyler Jenkins, M.D. ${ }^{2}$, Joseph Maslak, M.D. ${ }^{3}$, Alpesh Patel, MD, FACS
}

\section{${ }^{1}$, Chicago , Il, ${ }^{2}$ Northwestern University - Feinberg School of Medicine, Chicago, IL, ${ }^{3}$ Northwestern University - Feinberg School of} Medicine, Chicago, Il, ${ }^{4}$ Northwestern University, Chicago, IL

Background/Introduction: Surgical risk calculators exist in many fields and may assist in the identification of patients at increased risk for complication and readmissions. The American College of Surgeons (ACS) NSQIP risk calculator was developed from a cohort of over 1.4 million patients. The risk calculator uses 21 patient predictors (e.g., age, ASA class, BMI) and the planned procedure (CPT code) to predict the chance that patients will have any of 12 different outcomes (e.g. death, serious complication, reoperation) within 30-days following surgery. The purpose of this study is to determine if the ACS NISQIP risk calculator can predict 30-day complications after lumbar fusion.

Materials/Methods: A retrospective chart review was performed on patients that underwent primary lumbar fusion between Jan 2009-2015 at a single-institution, utilizing lumbar fusion CPT codes. Patients without 30 days post-operative follow-up were excluded. Descriptive statistics were calculated for the overall sample and single v. multi-level fusion. Logistic regression models were fit with actual complication occurrence as the dependent variable in each model and ACS estimated risk as the independent variable. The c-statistic was used as the measure of concordance for each model. Acceptable concordance was set at $\mathrm{c}>0.80$.

Results: A total of 237 patients were included in the analyses. Average age was 65 years and average BMI of 29.4 . 49\% of patients had 1 level fusions followed by $23.6 \%$ having 2 level fusions. Risk estimates for serious complications and any complications were found to be 6.9 ad 7.6 , respectively. Because there were no deaths, no models were fit for mortality. The logistic regression results for the overall sample are in Table 1. Because there were no deaths or UTIs, no models were fit for these outcomes. Only PNA met the criteria for c $>0.80$ for acceptable concordance.

Discussion/Conclusion: The ACS risk-calculator only predicted complications in the category of "discharge to skilled nursing facility" $(\mathrm{p}<0.001)$. However, the ACS risk calculator was unable to accurately predict specific complications on a more granular basis. The ACS risk calculator may be useful in the development of new institutional strategies for lumbar spinal fusion but does not provide accurate information for individual patient care.

RF24. Proposing a validated web-based tool predicting outcomes following initial hospitalization in patients undergoing lumbar arthrodesis for degenerative disc disease 
Piyush Kalakoti, $M D^{1}$, Nathan Hendrickson, $M D, M S^{2}$, Pawin Gajaseni, MD ${ }^{3}$, Luca Labianca, MD ${ }^{1}$, Christian Bowers, MD ${ }^{4}$, Meic Schmidt, $M D^{4}$, Andrew Pugely, $M D^{5}$; Alan Shamrock, $M D^{3}$

${ }^{1}$ University of Iowa, Iowa City, IA, ${ }^{2}$ Department of Orthopedics and Rehabilitation, University of Iowa, Iowa City, IA, ${ }^{3}$ University of Iowa, Iowa City, Iowa, ${ }^{4}$ New York Medical College, Westchester Medical Center \& Health Network, Valhalla, NY, ${ }^{5}$, New York, NY

Background/Introduction: Benchmarking outcomes and individualized risk-prediction are central in patient-oriented shared decision-making and optimization of surgical outcomes. Outcomes during initial hospitalization are critical in assessing value of care delivered, and are known to impact readmission rates. We attempted to create a predictive model of complications in patients undergoing lumbar arthrodesis for degenerative disc disease (DDD) and propose a web-based clinical tool for outcome prediction.

Materials/Methods: Using data obtained from the National Inpatient Sample (NIS), we identified adult patients undergoing lumbar arthrodesis for DDD. Multivariable regression techniques using generalized estimating equations were employed to create a predictive model of complications [discharge to facility, neurological complications, length of hospital stay, venous thromboembolism, cardiac and wound complication]. Internal validation of all models was performed by evaluating the impact on c-statistics using a 1000-bootstrapped replacement samples.

Results: The model development utilized 126,044 patients (median age:55 years; $42 \%$ male). Observed clinical outcomes include $0.1 \%$ casefatality, $14.5 \%$ discharge-to-facility, $2.2 \%$ patients with neurologic complications that included dural tears (CSF leak) and nerve root injury, $2.0 \%$ VTE, $1.5 \%$ respiratory complications, $1.0 \%$ ARF, $1.5 \%$ wound complications and $0.4 \%$ wound infections, $2.5 \%$ GI, $0.8 \%$ cardiac complication LOS (median:3 days). Multivariable analysis identified risk factors independently associated with the above outcomes (Figure 1: For discharge-to-facility). A validated web-based version of model for outcome prediction based on patient and hospital characteristics was developed, and can be accessed at the following link: https://www.neurosurgerycost.com/calc/lumbarspine

Discussion/Conclusion: The study quantifies risk estimates associated with poor initial outcomes in patients undergoing lumbar arthrodesis for DDD, and proposes a validated web-based tool(app) that could potentially be utilized by patients, providers and policy makers to assess individualized risks, shared decision making and guiding the process of patient counselling and informed consent.

\section{RF25. The national impact of spinal laminectomy readmissions throughout the United States}

Joseph Laratta, $M D^{1}$, Jamal Shillingford, $M D^{1}$, Abiola Atanda, $M D^{2}$, Joseph Lombardi, $M D^{1}$, Comron Saifi, MD ${ }^{3}$, Hemant Reddy, BS ${ }^{4}$, Lawrence Lenke, $M D^{5}$, Ronald Lehman, $M D^{6}$

${ }^{1}$ The Spine Hospital at Columbia University, New York, NY, ${ }^{2}$ Norton Leatherman Spine Center, Louisville, KY, ${ }^{3}$ Midwest Orthopedics at Rush, Chicago, IL, ${ }^{4}$ Northeast Ohio Medical University, Rootstown, $\mathrm{OH},{ }^{5}, \mathrm{New}$ York, $\mathrm{NY},{ }^{6}, \mathrm{New}$ York, $\mathrm{NY}$

Background/Introduction: Laminectomy is one of the most commonly performed procedures in spine surgery. In order to evaluate healthcare utilization and allocation of resources, it is essential to understand the demographic and economic data surrounding readmissions associated with laminectomy procedures performed in the U.S.

Materials/Methods: The Healthcare Cost and Utilization Project Nationwide Readmissions Database (NRD) was queried for patients undergoing a spinal laminectomy procedure during the years of 2009-2014. The NRD database includes discharges for patients with and without repeat hospital visits in a year and is weighted to provide national estimates. Demographic and economic data were obtained which included the age, sex, insurance type, location, frequency of readmission and cost per stay.

Results: A total of 396,600 index stays involved a laminectomy during 2014. An estimated 25,440 patients required readmission within 30 days for any cause $(6.4 \%)$. The readmission rate was constant over the study period (range, 6.3-6.5). The mean cost per stay for the index procedure increased $24.0 \%$ from $\$ 19,339$ in 2009 to $\$ 23,983$ in 2014 . The mean cost per stay for the readmission increased $19.3 \%$ from $\$ 13,005$ in 2009 to $\$ 15,512$ in 2014. The greatest number of readmissions occurred in patients older than 65 years $(8.0 \%)$. Based on insurance status, Medicare and Medicaid were more likely to be readmitted than private payers ( $8.2 \%$ versus $8.1 \%$ versus $4.5 \%$, respectively). When stratifying by median income for patient zip code, there was no difference in the rate of readmission across all groups.

Discussion/Conclusion: Over the past six years, the rate of readmission for spinal laminectomy procedures has remained constant, while the cost of index and readmission stays has increased significantly. Readmissions were more likely in patients over the age of 65 and in patients with Medicaid insurance.

RF26. Regional BMD differences measured by QCT: Is the clinically used L1/L2 average representative of the entire lumbosacral spine?

Stephan Salzmann, $M D^{1}$, Toshiyuki Shirahata, $M D^{1}$; Courtney Ortiz Miller, BA ${ }^{2}$, Brandon Carlson, $M D, M P H^{1}$, John Carrino, $M D^{1}$, Jingyan Yang, $M H S^{2}$, Andrew Sama, $M D^{l}$, Frank Cammisa, $M D^{l}$, Federico Girardi, $M D^{3}$, Alexander Hughes, $M D^{l}$

\section{${ }^{1}$ Hospital for Special Surgery, New York, New York, ${ }^{2}$ Hospital for Special Surgery, New York, New York, ${ }^{3}$, New york, New York}

Background/Introduction: Quantitative Computed Tomography (QCT) of the lumbar spine is used as an alternative to DXA in assessing bone mineral density (BMD). Clinically, a BMD average of L1/L2 is commonly used as a surrogate marker for overall bone health. However, it is unclear if this L1/L2 average is representative of the entire lumbosacral spine. The aim of this study was to assess the agreement between the clinically used L1/L2 BMD average and the other lumbosacral levels. 
Materials/Methods: Patients that underwent posterior lumbar spinal fusion from 2014 to 2017 with available preoperative lumbar spine CT imaging were included. Standard QCT measurements at L1/L2 and additional experimental measurements of L3-S1 were performed. The correlations between L1/L2 average and the other vertebral bodies of the lumbosacral spine (L3, L4, L5, S1) were evaluated.

Results: 296 patients (55.4\% female; mean age 63.1 years) met inclusion criteria. The vertebral BMD values showed a gradual decrease from L1 to $\mathrm{L} 3$ and subsequently increased from L4 to $\mathrm{S} 1(\mathrm{~L} 1=118.8 \mathrm{mg} / \mathrm{cm} 3, \mathrm{~L} 2=116.6 \mathrm{mg} / \mathrm{cm} 3, \mathrm{~L} 3=112.5 \mathrm{mg} / \mathrm{cm} 3, \mathrm{~L} 4=122.4 \mathrm{mg} / \mathrm{cm} 3, \mathrm{~L} 5=135.3$ $\mathrm{mg} / \mathrm{cm} 3, \mathrm{~S} 1=157.4 \mathrm{mg} / \mathrm{cm} 3$ ) (Figure 1). Our data showed strong correlation between the L1/L2 average and the average of the other lumbosacral vertebrae (L3-S1) with a Pearson's correlation coefficient, $\mathrm{r}=0.85$. We also analyzed the correlation between the L1/L2 average and each individual lumbosacral vertebra. Similar relationships were observed ( $\mathrm{r}$ value, $0.67-0.87$ ) with the strongest correlation between the L1/L2 average and L3.

Discussion/Conclusion: Our data demonstrates considerable regional BMD throughout the lumbosacral spine. Nevertheless there is high concordance between the clinically-used L1/L2 BMD average and the other lumbosacral vertebrae. As the closest vertebra to the standard measurement site, L3 showed the strongest individual correlation to the L1/L2 average. Although still strongly correlated, the Pearson's correlation coefficients were smaller for the L4, L5 and S1 vertebra. This might be of importance, since the majority of patients in our cohort underwent fusion procedures at L4-5 (71.3\%) and/or L5-S1 (44.9\%). In summary, the L1/L2 BMD average is a useful bone quantity measure of the entire lumbosacral spine in patients undergoing posterior lumbar spinal fusion.

RF27. Bone marrow concentration vs. iliac crest bone graft: 2-year results in a single-blinded randomized controlled trial on thoracolumbar spinal fusion bone grafts in multi-level adult spinal deformity

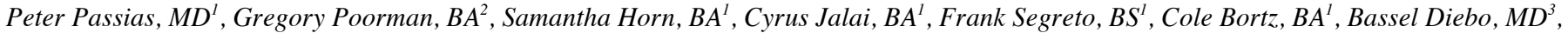
Themistocles Protopsaltis, $\mathrm{MD}^{4}$; Michael Gerling, $\mathrm{MD}^{5}$, Thomas Errico, $\mathrm{MD}^{6}$

${ }^{1}$, New York, $N Y,{ }^{2}$, bronxville, ny, ${ }^{3}$, Brooklyn, NY, ${ }^{4}$ Department of Orthopedic Surgery, NYU Langone Orthopedic Hospital, NYU Langone Health, Manhattan, NY, ${ }^{5}$, TriBeCa, NY, ${ }^{6}$ Department of Orthopedic Surgery, NYU Langone Orthopedic Hospital, NYU Langone Health, New York, New York

Background/Introduction: Though iliac crest bone grafts (ICBG) during posterior lumbar fusions represents the gold standard, there are complications associated with its use (donor site morbidity, increased operative time, added blood loss). Bone marrow aspirate concentrate (BMC) and allograft has produced promising results in orthopaedic trauma procedures and single-level spinal fusions, with reported similar osteoconductive and osteoinductive effects as ICBG. This study compares the efficacy of BMC+allograft to ICBG for multi-level posterior lumbar or thoracolumbar fusions.

Materials/Methods: This study was an single institution, prospective, single-blinded randomized controlled trial. Inclusion Criteria: Patients undergoing open posterior lumbar fusions, ages $>18$ years. Patients were randomized and enrolled in a 2:1 ratio: BMC(2) or ICBG(1). Full-length radiographs and health-related-quality-of-life (HRQoL) scores at baseline, 6-weeks, 3-months, 6-months, 1-year, and 2-years post-operative were obtained, as well as 1-year post-operative CT-scans. HRQoL metrics included the Oswestry-Disability-Index(ODI), Short-Form-12(SF-12), and numeric-pain-rating-scale. CT and radiographic fusion assessment was performed by an independent radiologist.

Results: 27 patients were included (17 BMC, 10 ICBG). Mean age was 56 years, $48 \%$ of patients were smokers, and $40.7 \%$ of patients had previous spinal surgery. Average levels-fused: 6.0 [range: 2-23] for BMC, 6.23 [range 2-8] for ICBG, p=0.935. EBL (average 2231ml) and OR-time (average $8.02 \mathrm{hrs}$ ) were similar between groups, $\mathrm{p}>0.05$. No significant intraoperative complications were related to BMC or ICBG protocol. Within the 2-year follow-up period, there were 7 complications in the BMC group (41.1\%) and 4 in the ICBG group (40\%). ICBG patients had greater incidence of rod breakage (BMC: 0\% vs. ICBG: $25 \%, \mathrm{p}=0.024$ ). $88.2 \%$ of BMC patients were graded 'fused' at 1-year, and 60\% of ICBG patients $(\mathrm{p}=0.088) .5 .9 \%$ of BMC patients were graded 'bilateral pseudarthrosis', and $30 \%$ of ICBG patients $(\mathrm{p}=0.088)$. An average 2 billion 60 million nucleated cells were delivered per patient. This did not show correlation with fusion score $(\mathrm{p}=0.742)$.

Discussion/Conclusion: Conclusion: In long fusions for adult spinal deformity, where autologous graft is limited, bone marrow concentrated via centrifuge is a viable alternative to iliac crest bone graft. The current study found a decrease in hardware failures in patients with BMC compared to ICBG, and a trend towards better fusion scores.

RF28. The "kickstand rod" technique for correction of coronal imbalance in patients with adult spinal deformity: theory and technical considerations

Melvin Makhni, $M D M B A^{1}$, Ying Zhang, $M D^{2}$, Meghan Cerpa, $M P H^{2}$, Suthpias Pongmanee, $M D^{2}$, Ming Yang, $M D^{2}$, Lawrence Lenke, $M D^{2}$

\section{${ }^{l}$ The Spine Hospital, Columbia University, New York City, NY, ${ }^{2}$, New York, NY}

Background/Introduction: The objective of this study is to describe and evaluate a new surgical procedure for correction of coronal imbalance (CI) in adult spinal deformity (ASD) patients, called the "kickstand rod" technique.

Materials/Methods: The records were reviewed of 20 consecutive patients with Adult Spinal Deformity and coronal imbalance who had surgery between July 2015 and June 2017 who were each treated with a long-segment fusion and a kickstand rod. For the kickstand rod technique, an iliac screw was placed on the side of the trunk shift and connected proximally through a side-by-side domino link to the thoracolumbar junction; this rod was distracted to promote coronal plane balancing. Distraction occurred with the contralateral side locked in order to preserve sagittal correction. Radiographic and clinical analysis was conducted to evaluate the outcomes and possible complications of the kickstand rod technique.

Results: The mean age of the patients was 52.25 years (range 14-73). Sixteen of the twenty patients were female. Coronal imbalance preoperatively was on average $58.28 \mathrm{~cm}$; post-operatively before discharge from the hospital this value was improved to $18.32 \mathrm{~cm}$. On final follow-up 
at an average of 12.65 months (range 2.34 - 24.80 months), coronal imbalance was $13.10 \mathrm{~cm}$, resulting in an absolute mean final improvement in CI of $45.18 \mathrm{~cm}$. There were no neurologic, vascular or implant-related complications in any of the patients. Two patients developed wound dehiscence that was successfully treated without implant removal and one developed PJK requiring extension of the construct proximally. There were no complaints about screw prominence, kickstand construct implant failure, or significant worsening of coronal imbalance after surgery.

Discussion/Conclusion: The "kickstand rod" technique is a safe and effective method for correction of coronal imbalance in ASD patients with or without pelvic obliquity. This technique was found to provide marked coronal correction, as well as provide additional strength to the overall construct without adverse consequence.

RF29. Can S2-alar-iliac (S2AI) screws be placed accurately without fluoroscopy? $M D^{2}$

Daniel Kang, $M D^{1}$, Jacob Buchowski, MD ${ }^{2}$, Lawrence Lenke, $M D^{3}$, Panya Luksanapruska, MD ${ }^{4}$, Ronald Lehman, MD ${ }^{5}$, Jamal McClendon, Jr.,

\author{
${ }^{1}$ Madigan Army Medical Center, Tacoma, WA, ${ }^{2}$ Washington University School of Medicine, St. Louis, MO, ${ }^{3}, \mathrm{New}$ York, NY, ${ }^{4}$ Department of \\ Orthopedic Surgery, Faculty of Medicine Siriraj Hospital, Bangkoknoi, Bangkok, ${ }^{5}$, New York, NY
}

Background/Introduction: S2AI screw insertion is most often performed with fluoroscopic guidance, however, the freehand S2AI screw insertion provides an alternative technique avoiding radiation exposure. However, the accuracy and safety of the freehand S2AI screw technique has not been completely evaluated.

Materials/Methods: Retrospective analysis of adult spinal deformity patients treated at one institution, single surgeon over 4 years (2010-14) with freehand S2AI screw placement, consecutively assessed with intraoperative computed tomography (CT) scan. We analyzed intraoperative CT images of patients who underwent freehand S2AI screw fixation. Each CT image sequence was assessed by 3 spine surgeons, and any pedicle awl or screw violation of the cortical wall was confirmed/graded through consensus agreement. S2AI screw position was graded as acceptable (complete screw within the pelvic/iliac corridor), minor violation $(<=6 \mathrm{~mm})$ and major/critical violation $(>6 \mathrm{~mm})$. Screws not adequately visualized on the CT images were excluded.

Results: Overall, there were 190 freehand S2AI screws inserted in 98 patients ( $\mathrm{n}=93$ Left; $\mathrm{n}=97$ Right) and adequately visualized on intraoperative CT images. There were 5 patients with Right S2AI + Left Iliac(3 L lateral pedicle awl violations), 1 patient Left S2AI + Right iliac screw, and 5 patients with multiple $(>2)$ ipsilateral pelvic fixation points. Overall there was a $9.5 \%$ violation rate $(n=8 \mathrm{Left} ; \mathrm{n}=10 \mathrm{Right})$. The rate of minor violation was $5.8 \%$ (lateral $n=9,4.7 \%$, average $3.1 \mathrm{~mm}$; medial $\mathrm{n}=2,1.1 \%$, average $2.5 \mathrm{~mm}$ ), and major/critical violation was $3.2 \%$ (lateral $\mathrm{n}=3$, $1.6 \%$, average $10.8 \mathrm{~mm}$; medial $\mathrm{n}=4,2.1 \%$, average $15.6 \mathrm{~mm})$. There were 12 pedicle awl violations, all were lateral ( $\mathrm{n}=8 \mathrm{Left} ; \mathrm{n}=4 \mathrm{Right})$. No patients had neurologic, vascular, or visceral complications related to S2AI screw placement.

Discussion/Conclusion: Freehand S2AI screw insertion is a safe and reliable method. Our study found a $90.5 \%$ accuracy rate for acceptable screw position using freehand S2AI screw insertion without fluoroscopy, with 5.8\% minor and 3.2\% major/critical cortical wall violations. There were no S2AI screw related complications, and most screws violations were minor and occurred laterally. We found freehand S2AI screw insertion has excellent accuracy, and obviates the use of fluoroscopic guidance.

\title{
RF30. The role of pelvic parameters on S2 alar-iliac (S2AI) screw trajectory
}

Jamal Shillingford, $M D^{1}$, Joseph Laratta, $M D^{1}$, James Lin, $M D, M S^{2}$, Joseph Lombardi, $M D^{1}$, Lawrence Lenke, MD ${ }^{3}$, Ronald Lehman, $M D^{4}$

\author{
${ }^{1}$ The Spine Hospital at Columbia University, New York, NY, ${ }^{2}$ Columbia University Medical Center, New York, NY, ${ }^{3}$, New York, NY, ${ }^{4}$, New \\ York, NY
}

Background/Introduction: Spinopelvic fixation utilizing S2AI screws provides optimal fixation across the lumbosacral junction allowing for solid fusion, especially in long segment fusion constructs. Freehand placement of such screws relies heavily on the rich surrounding sacropelvic anatomy.

Materials/Methods: The medical records and preoperative CT scans of 33 consecutive patients with degenerative lumbar pathology between 2015-2016 were reviewed by two independent investigators. Preoperative standing X-rays were assessed to measure pelvic parameters including sacral slope, pelvic tilt, and pelvic incidence. Using 3-dimensional CT reconstructions, an ideal S2AI trajectory was defined as a start point between the S1 and S2 foramen with screw axis directed towards the anterior-inferior iliac spine on the sagittal plane. In the axial plane, the trajectory started at the lateral aspect of the S1/2 foramen and was directed through the narrowest portion of ilium. Sacral slope, horizontal angle, sagittal angle, intrasacral distance and estimated screw lengths were recorded.

Results: The mean age at the time of surgery was $62.4+-12.5$ years and there were $14(42.4 \%)$ female patients in the cohort. The average sagittal angle measured in the sagittal plane was $27.3+-4.1^{\circ}$. The average horizontal angle measured in the axial plane using the posterior superior iliac spine as a reference was $35.9+-3.9^{\circ}$. Maximum screw length and intra-sacral screw length were $109.7+-16.4 \mathrm{~mm}$ and $33.6+-6.4 \mathrm{~mm}$ respectively. Pelvic tilt was found to have a moderate inverse correlation with sagittal screw trajectory $(\mathrm{r}=-0.467, \mathrm{p}$-value $=0.006)$. Pelvic incidence and sacral slope had weak correlations with sagittal screw angle. In the subgroup analysis, patients with high pelvic tilt $>20^{\circ}$ had a significantly lower sagittal screw trajectory compared to those with a normal pelvic tilt $\left(24.9+-3.7^{\circ}\right.$ versus $29.8+-2.8^{\circ}$, p-value $\left.=<0.001\right)$.

Discussion/Conclusion: In this study, we found an inverse relationship between increased pelvic tilt and lower S2AI screw trajectory in the sagittal plane. This understanding of the pelvic parameters is imperative to safe and accurate placement of S2AI screws. 
Disclaimer: The Journal of Neurosurgery Publishing Group (JNSPG) acknowledges that the preceding abstracts are published as submitted and did not go through JNSPG's peer-review or editing process. 